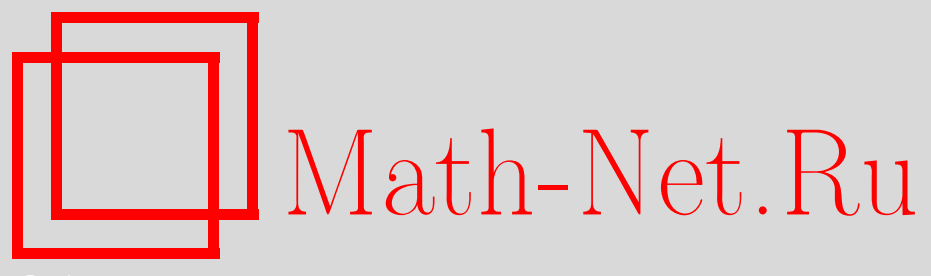

А. В. Устинов, Трехмерные цепные дроби и суммы Клостермана, УМH, 2015, том 70, выпуск 3, 107-180

DOI: https://doi.org/10.4213/rm9637

Использование Общероссийского математического портала Math-Net.Ru подразумевает, что вы прочитали и согласны с пользовательским соглашением http://www . mathnet.ru/rus/agreement

Параметры загрузки:

IP: 34.229 .108 .108

26 апреля 2023 г., 18:24:56

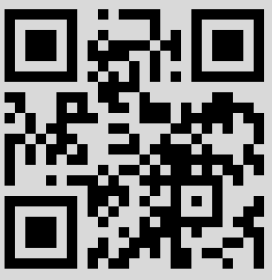




\title{
Трехмерные цепные дроби и суммы Клостермана
}

\begin{abstract}
А. В. Устинов
Обзор посвящен результатам, связанным с метрическими свойствами классических цепных дробей и трехмерных цепных дробей Вороного-Минковского. Основное внимание уделяется применению аналитических методов, основанных на оценках сумм Клостермана. В статье развивается аппарат, предназначенный для решения задач на трехмерных решетках. В основе подхода лежит идея редукции к предыдущей размерности, применявшаяся ранее Линником и Скубенко при исследовании целочисленных решений детерминантного уравнения $\operatorname{det} X=P$, где $X-$ матрица размера $3 \times 3$ с независимыми коэффициентами и $P$ - растущий параметр.

Предлагаемый метод применяется для изучения статистических свойств трехмерных цепных дробей Вороного-Минковского в решетках с фиксированным определителем. В частности, для среднего числа базисов Минковского доказывается асимптотическая формула со степенным понижением в остаточном члене. Этот результат можно считать трехмерным аналогом теоремы Портера о средней длине конечных цепных дробей.

Библиография: 127 названий.
\end{abstract}

Ключевые слова: трехмерные цепные дроби, решетки, суммы Клостермана, статистики Гаусса-Кузьмина.

DOI: $10.4213 / \mathrm{rm} 9637$

СоДЕРЖАНИЕ

1. Введение............................................. 108

1.1. Проблема Линника................................... 108

1.2. Редукция Линника-Скубенко . . . . . . . . . . . . . . . . . . . . . . . . 109

1.3. Теоремы Хейльбронна и Портера....................... 111

1.4. Краткая формулировка основного результата .............. 111

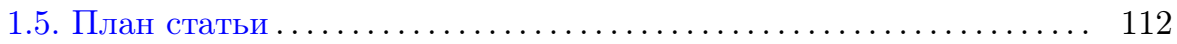

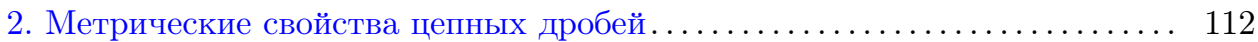

2.1. Мера Гаусса ............................... 112

2.2. Статистические свойства конечных цепных дробей........... 114

2.3. Вполне примитивные решетки .................... 115

2.4. Многомерный аналог меры Гаусса.................. 116

Работа выполнена при поддержке РФФИ (грант № 14-01-90002) и фонда Д. Зимина “Династия".

(C) А.В. Устинов, 2015 


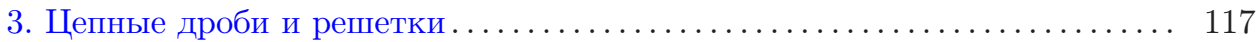

3.1. Геометрия цепных дробей ......................... 117

3.2. Базисы Минковского .................................. 119

3.3. Трехмерные цепные дроби . . . . . . . . . . . . . . . . . . 120

3.4. Формулировка основного результата................... 126

4. Двумерный случай как модельная задача...................... 128

4.1. Постановка задачи ................................ 128

4.2. Разбиение на случаи . . . . . . . . . . . . . . . . . . . . . . . . . . . . . 129

4.3. Линейная параметризация решений .................... 130

4.4. Первый вариант оценки остаточного члена................. 130

4.5. Второй вариант оценки остаточного члена ................ 131

4.6. Оценка итогового остатка .......................... 132

4.7. Подсчет главного члена............................ 133

5. Разбиение на случаи . . . . . . . . . . . . . . . . . . . . . . . . . 133

5.1. Приведенные матрицы . . . . . . . . . . . . . . . . . . . 133

5.2. Свойства построенного разбиения .................. 136

5.3. Схема доказательства основного результата ................ 138

5.4. Различные варианты сумм Клостермана ................. 140

6. Первый вариант оценки остатка ........................ 140

6.1. Линейная параметризация решений .................... 140

6.2. Переход к интегрированию в третьей строке .............. 142

6.3. Переход к интегрированию во второй строке .............. 143

7. Второй вариант оценки остатка........................... 148

8. Третий вариант оценки остатка ......................... 156

9. Завершение доказательства основного результата ............... 161

9.1. О приведенных базисах в плоских решетках............... 161

9.2. Оценка остаточного члена . . . . . . . . . . . . . . . . . . 163

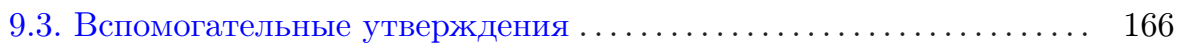

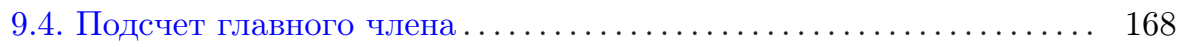

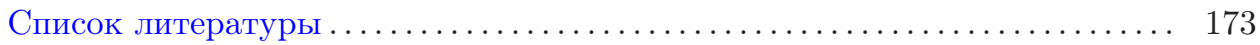

\section{1. Введение}

1.1. Проблема Линника. Многие теоретико-числовые задачи сводятся к изучению диофантова уравнения

$$
F\left(x_{1}, \ldots, x_{n}\right)=P
$$

где $F$ - однородный многочлен и $P$ - целое число. Единственным общим методом, позволяющим описывать асимптотические свойства решений уравнения (1.1), является круговой метод Харди-Литтлвуда, который, однако, требует дополнительных свойств от многочлена $F$ (см. [1]). В некоторых ситуациях распределение решений уравнения (1.1) удается исследовать методами алгебраической теории чисел и алгебраической геометрии (см. [2], [3]), однако в общем случае для числа решений не известно даже правильных по порядку оценок. 
Если уравнение (1.1) задает однородное многообразие с действием линейной алгебраической группы, то появляется возможность применять методы гармонического анализа на этой группе (см. [4]-[7]). Важным частным случаем такой ситуации является детерминантное уравнение

$$
\operatorname{det} X=P \text {, }
$$

где $X$ - квадратная матрица с независимыми коэффициентами. Для матриц размера $3 \times 3$ в работе [8] (см. также [9; гл. VIII]) Линник и Скубенко доказали, что при $P \rightarrow \infty$ целочисленные решения уравнения (1.2) равномерно распределены по мере Хаара. Они решали задачу в предположении, что нормированная матрица $\tilde{X}=X P^{-1 / 3}$ содержится в некоторой фиксированной области $\Omega \subset S L_{3}(\mathbb{R})$ конечной меры. Для числа решений уравнения (1.2) Линник и Скубенко доказали асимптотическую формулу без явного указания понижения в остаточном члене. (Явная оценка остаточного члена для области $\|\widetilde{X}\| \leqslant 1$, где $\|$ · $\|$ - евклидова норма, дана в [10].) В общем случае задача о распределении целочисленных решений уравнения (1.1) известна как проблема Линника и также обычно рассматривается в предположении, что $\widetilde{X}=X P^{-1 / d} \ll 1$, где $d-$ степень многочлена $F$ (см. [4], [5], [10]). Развитие метода Линника и Скубенко см. в работах [11]-[14].

1.2. Редукция Линника-Скубенко. Согласно критерию Вейля (см., например, [15]) необходимым и достаточным условием равномерного распределения системы функций $\left(f_{1}(x), \ldots, f_{n}(x)\right)$ является выполнение равенства

$$
\sum_{x=1}^{P} e\left(m_{1} f_{1}(x)+\cdots+m_{n} f_{n}(x)\right)=o(P) \quad(P \rightarrow \infty),
$$

где $m_{1}, \ldots, m_{n}$ - произвольные целые числа, не равные одновременно нулю. Это условие позволяет сводить исследование равномерного распределения систем функций к оценкам соответствующих тригонометрических сумм.

Многие задачи, связанные с плоскими целочисленными решетками (см. подробнее в п. 2.2), сводятся к исследованию решений детерминантного уравнения

$$
\operatorname{det}\left(\begin{array}{ll}
a_{1} & a_{2} \\
b_{1} & b_{2}
\end{array}\right)=P
$$

Это уравнение можно заменить эквивалентным сравнением

$$
a_{2} b_{1}+P \equiv 0 \quad(\bmod a)
$$

предполагая, что $a_{1}=a>0$ фиксировано, а значение $b_{2}$ находится из равенства $b_{2}=\left(a_{2} b_{1}+P\right) a^{-1}$.

С помощью критерия Вейля задача о равномерном распределении решений сравнения (1.4) сводится к оценкам сумм

$$
K_{a}(m, n, q)=\sum_{x, y=1}^{a} \delta_{a}(x y+q) e\left(\frac{m x+n y}{a}\right)
$$


где $m, n, q$ - произвольные целые, $a$ - натуральное, $e(t)=e^{2 \pi \sqrt{-1} t}$ и $\delta_{a}$ - характеристическая функция делимости на $a$ :

$$
\delta_{a}(x)= \begin{cases}1, & \text { если } x \equiv 0(\bmod a), \\ 0, & \text { если } x \neq 0(\bmod a) .\end{cases}
$$

При $q=-1$ суммы (1.5) совпадают с классическими суммами Клостермана

$$
K_{a}(m, n)=\sum_{x, y=1}^{a} \delta_{a}(x y-1) e\left(\frac{m x+n y}{a}\right) .
$$

Для сумм (1.6) и (1.5) известны нетривиальные оценки, что позволяет находить асимптотические формулы для сумм вида

$$
\sum_{a_{1} b_{2}-a_{2} b_{1}=P} f\left(a_{1}, a_{2}, b_{1}, b_{2}\right),
$$

заменяя их соответствующими интегралами. $\mathrm{K}$ вычислению подобных сумм сводятся задачи из геометрии чисел, теории цепных дробей и т.д. (см. обзор [16]).

В трехмерном случае базисы решеток с определителем $P$ параметризуются решениями детерминантного уравнения (1.2), где $X$ - матрица вида

$$
X=\left(\begin{array}{lll}
a_{1} & a_{2} & a_{3} \\
b_{1} & b_{2} & b_{3} \\
c_{1} & c_{2} & c_{3}
\end{array}\right)
$$

в которой координаты базисных векторов записаны по столбцам. При изучении трехмерных цепных дробей Вороного-Минковского (см. оригинальные публикации [17], [18] и [19], а также их переизложение в [20], [21] и [22]) возникает необходимость в подсчете решений уравнения (1.2), для которых нормированная матрица $\widetilde{X}=X P^{-1 / 3}$ может меняться в области $\Omega \subset S L_{3}(\mathbb{R})$ бесконечной меры.

Метод Линника-Скубенко был основан на редукции к предыдущей размерности - детерминантному уравнению (1.3). Основная идея заключалась в том, что если матрица $\left(\begin{array}{ll}a_{1} & a_{2} \\ b_{1} & b_{2}\end{array}\right)$ фиксирована, имеет ненулевой определитель $q$ и $(q, P)=1$, то по каждому решению (1.8) уравнения (1.2) можно построить серию решений

$$
\left(\begin{array}{ccc}
a_{1} & a_{2} & z a_{3}+s a_{1}+t a_{2} \\
b_{1} & b_{2} & z b_{3}+s b_{1}+t b_{2} \\
z^{-1} c_{1}+u a_{1}+v b_{1} & z^{-1} c_{2}+u a_{2}+v b_{2} & *
\end{array}\right)
$$

где $z \in \mathbb{Z}_{q}^{*}, z z^{-1} \equiv 1(\bmod q)$, а $s, t, u, v$ - произвольные целые. Наличие параметра $z$, нелинейно входящего в параметризацию (1.9), позволяет с помощью сумм Клостермана свести задачу о распределении решений уравнения (1.2) к суммам вида (1.7), методы вычисления которых хорошо известны. 
В статье [23] была предложена более точная версия редукции Линника-Скубенко, применимая, в частности, для областей $\Omega$ бесконечного объема. Возникающие после редукции вспомогательные двумерные задачи были решены в [24]. В настоящей статье результаты работ [23] и [24] применяются к исследованию статистических свойств трехмерных цепных дробей Вороного-Минковского. Можно ожидать, что предлагаемый подход окажется полезным и при решении других задач, связанных с трехмерными решетками.

1.3. Теоремы Хейльбронна и Портера. Для рационального $r$ через $l(r)$ будем обозначать длину разложения $r$ в конечную цепную дробь

$$
r=\left[a_{0} ; a_{1}, \ldots, a_{l}\right]=a_{0}+\frac{1}{a_{1}+\ddots+\frac{1}{a_{l}}},
$$

где $a_{0}=\lfloor r\rfloor$ (целая часть $\left.r\right), a_{1}, \ldots, a_{l}$ - натуральные и $a_{l} \geqslant 2$ при $l \geqslant 1$.

В статье [25] Хейльбронн доказал асимптотическую формулу для среднего значения $l(r)$, взятого по рациональным числам $r$ с фиксированным знаменателем:

$$
\frac{1}{\varphi(P)} \sum_{a=1}^{P} l\left(\frac{a}{P}\right)=\frac{2 \log 2}{\zeta(2)} \log P+O\left((\log \log P)^{4}\right)
$$

(здесь и далее звездочка означает, что суммирование проходит по приведенной системе вычетов). Позднее Портер (см. [26]) уточнил этот результат, выделив следующий значащий член, являющийся абсолютной константой:

$$
\frac{1}{\varphi(P)} \sum_{a=1}^{P} l\left(\frac{a}{P}\right)=\mathscr{Q}_{1}(\log P)+O\left(P^{-1 / 6+\varepsilon}\right)
$$

(через $\mathscr{Q}_{m}(x)$ будем обозначать многочлен степени $m$ от переменной $x$; константы в знаках $O$ всегда будут предполагаться зависящими от $\varepsilon$ - сколь угодно малого положительного числа). Доказательство Хейльбронна элементарно. Портер использовал оценки сумм Клостермана и оценки тригонометрических сумм по ван дер Корпуту.

1.4. Краткая формулировка основного результата. В настоящей статье с помощью уточненной версии редукции Линника-Скубенко из работы [23] строится метод анализа минимальных базисов в трехмерных решетках. В частности, этот метод позволяет доказать для них трехмерный аналог результата Портера (1.11).

Теорема 1.1. Среднее число базисов Минковского по вполне примитивным решеткам с определителем $P$ имеет асимптотику

$$
\mathscr{Q}_{2}(\log P)+O\left(P^{-1 / 34+\varepsilon}\right) .
$$

Более точная формулировка этого результата будет дана ниже после определения всех необходимых понятий (см. теорему 3.3 ниже). С точки зрения 
проблемы Линника наличие в асимптотике многочлена второй степени от логарифма $P$ объясняется тем, что при подсчете решений уравнения $\operatorname{det} X=P$ приходится иметь дело с областью бесконечного объема на многообразии, заданном уравнением $\operatorname{det} \widetilde{X}=1$.

Трехмерный аналог теоремы Хейльбронна (1.10) был доказан Илларионовым в [27] (по поводу других многомерных обобщений см. [28]-[30]). Аргументы Илларионова позволяют выделить старший член в асимптотической формуле (1.12) с остатком $O(\log P \log \log P)$.

Равенство (1.11) можно понимать как формулу для средней длины алгоритма Евклида, примененного к паре чисел $(a, P)$ таких, что $1 \leqslant a \leqslant P$ и $(a, P)=1$. С геометрической точки зрения левую часть равенства (1.11) можно понимать как среднее число минимальных базисов в решетках с базисами из пары векторов $(1, a)$ и $(0, P)$. Формула $(1.12)$ описывает среднее число минимальных базисов в трехмерных решетках, порожденных векторами $(1,0, a),(0,1, b)$ и $(0,0, P)$ (см. п. 2.3). Эту же величину можно понимать как среднее число всевозможных базисов, которые могут возникнуть при работе алгоритма Евклида, примененного к тройке $(a, b, P)$, в которой $1 \leqslant a, b \leqslant P$ и $(a, P)=(b, P)=1$.

1.5. План статьи. В разделе 2 дается краткий обзор результатов, связанных с метрической теорией бесконечных и конечных цепных дробей.

В разделе 3 рассказывается о трехмерных цепных дробях по Вороному и Минковскому. Приводится точная формулировка основного результата.

Чтобы проиллюстрировать схему доказательства основной теоремы, в разделе 4 коротко описаны основные шаги, необходимые для решения модельной задачи - доказательства упрощенной версии равенства (1.11). Общая схема рассуждений при доказательстве основной теоремы 3.3 будет такой же.

В разделе 5 решения уравнения (1.2) разбиваются на группы, в каждой из которых решения будут подсчитываться (до некоторого момента) независимо. В конце п. 5.3 дается подробная схема доказательства основного результата.

В разделах 6-8 тремя разными способами доказываются асимптотические формулы для числа решений уравнения (1.2) с фиксированным угловым элементом $a_{1}$ и угловым минором $q=\left|\begin{array}{ll}a_{1} & a_{2} \\ b_{1} & b_{2}\end{array}\right|$.

В разделе 9 завершается доказательство основной теоремы 3.3.

\section{2. Метрические свойства цепных дробей}

2.1. Мера Гаусса. Метрическая теория цепных дробей восходит к задаче Гаусса о типичном поведении чисел вида

$$
\alpha_{n}=T^{n}(\alpha)=\left[0 ; a_{n+1}, a_{n+2}, \ldots\right]
$$

где $\alpha=\left[0 ; a_{1}, a_{2}, \ldots\right]$ - случайное число из интервала $[0,1)$ и $T$ - отображение Гaycca:

$$
T(\alpha)=\left\{\frac{1}{\alpha}\right\} \quad \text { при } \alpha \neq 0, \quad T(0)=0 .
$$


Для действительного $\xi \in[0,1]$ обозначим через $F_{n}(\xi)$ меру множества чисел $\alpha \in[0,1)$, для которых $\alpha_{n} \leqslant \xi$. Исследуя итерации отображения $T$, Гаусс пришел к предположению, что

$$
\lim _{n \rightarrow \infty} F_{n}(\xi)=\log _{2}(1+\xi)=\frac{\log (1+\xi)}{\log 2}
$$

(об этом известно из переписки Гаусса с Лапласом, см. [31; гл. 3]). Кузьмин в работе [32] получил асимптотическую формулу

$$
F_{n}(\xi)=\log _{2}(1+\xi)+O\left(e^{-\lambda \sqrt{n}}\right) \quad(\lambda>0),
$$

из которой следует гипотеза Гаусса. Результат Кузьмина уточнялся П. Леви [33] и Вирзингом [34]. Окончательное решение задачи Гаусса принадлежит Бабенко [35]. Он доказал существование бесконечной убывающей к нулю последовательности чисел $\lambda_{j}$,

$$
1>\left|\lambda_{1}\right|>\left|\lambda_{2}\right| \geqslant \cdots \geqslant\left|\lambda_{k}\right| \geqslant\left|\lambda_{k+1}\right| \geqslant \cdots,
$$

и соответствующей последовательности аналитических функций $\psi_{k}(\xi)$, для которых

$$
F_{n}(\xi)=\log _{2}(1+\xi)+\sum_{k=1}^{\infty} \psi_{k}(\xi) \lambda_{k}^{n} .
$$

Равенство (2.1) означает, что типичное поведение чисел $x_{n}=T^{n}(x)$ описывается мерой Гаусса

$$
d \nu(\xi)=\frac{1}{\log 2} \frac{d \xi}{1+\xi}, \quad \xi \in[0,1],
$$

инвариантной относительно отображения $T$. В частности, из этого следует, что вероятности появления натуральных чисел $k$ в качестве неполных частных у действительных чисел описываются распределением Гаусса-Кузьмина

$$
p_{k}=-\log _{2}\left(1-\frac{1}{(k+1)^{2}}\right), \quad k \geqslant 1 .
$$

Во многих случаях удобней рассматривать расширенную меру Гаусса

$$
d \bar{\nu}(\alpha, \beta)=\frac{1}{\log 2} \frac{d \alpha d \beta}{1+\alpha \beta}, \quad(\alpha, \beta) \in[0,1]^{2},
$$

инвариантную относительно обратимого почти всюду отображения (см. [36])

$$
\bar{T}:(\alpha, \beta) \rightarrow \begin{cases}\left(\left\{\frac{1}{\alpha}\right\},\left(\left[\frac{1}{\alpha}\right]+\beta\right)^{-1}\right) & \text { при } \alpha \neq 0 ; \\ (\alpha, \beta) & \text { при } \alpha=0 .\end{cases}
$$

Если разложить в цепные дроби координаты начальной точки $\left(\alpha_{0}, \beta_{0}\right)$ :

$$
\alpha_{0}=\left[0 ; a_{0}, a_{1}, \ldots\right], \quad \beta_{0}=\left[0 ; a_{-1}, a_{-2}, \ldots\right],
$$

то отображение $\bar{T}$ будет эквивалентно сдвигу на бесконечной в обе стороны последовательности $\left(\ldots, a_{-2}, a_{-1}, a_{0}, a_{1}, a_{2}, \ldots\right)$, полученной конкатенацией разложений $(2.5)$, записанных в разные стороны: $\bar{T}^{n}\left(\alpha_{0}, \beta_{0}\right)=\left(\alpha_{n}, \beta_{n}\right)$, где $n-$ произвольное целое, $\alpha_{n}=\left[0 ; a_{n}, a_{n+1}, \ldots\right]$ и $\beta_{n}=\left[0 ; a_{n-1}, a_{n-2}, \ldots\right]$. 
2.2. Статистические свойства конечных цепных дробей. Дискретный вариант проблемы Гаусса состоит в изучении статистических свойств конечных цепных дробей. Для рациональных чисел (так же как и для действительных) статистики Гаусса-Кузьмина удобней понимать в более широком смысле. Для действительных $\xi, \eta \in[0,1]$ и рационального $r$ определим статистики Гаусса-Кузьмина равенством

$$
l_{\xi, \eta}(r)=\sum_{j=1}^{s+1}\left[\left[0 ; a_{j}, \ldots, a_{s}\right] \leqslant \xi,\left[0 ; a_{j-1}, \ldots, a_{1}\right] \leqslant \eta\right] .
$$

Считаем, что пустая цепная дробь по определению равна нулю; если $A$ - некоторое условие, то $[A]$ - характеристическая функция множества, задаваемого этим условием: $[A]=1$, если условие выполнено, и $[A]=0$ в противном случае. В частности, $l_{1,1}(r)=l(r)$.

Распределение неполных частных в разложении чисел $a / b$ в случае, когда $1 \leqslant a \leqslant b \leqslant P$ и $P \rightarrow \infty$, впервые было изучено в 1961 г. Лохсом (см. [37], а также [25], [38]). Позднее эта задача в более общей формулировке ставилась Арнольдом [39; задача 1993-11] (задаче Арнольда посвящены статьи [40]-[45]). Результат Лохса можно интерпретировать следующим образом: для среднего значения статистик Гаусса-Кузьмина (2.6) справедлива асимптотическая формула

$$
\frac{2}{P^{2}} \sum_{1 \leqslant a \leqslant b \leqslant P} l_{\xi, \eta}\left(\frac{a}{b}\right)=\frac{2}{\zeta(2)} \int_{0}^{\xi} \int_{0}^{\eta} \frac{d \alpha d \beta}{(1+\alpha \beta)^{2}} \log P+C(\xi, \eta)+O\left(P^{-1 / 2+\varepsilon}\right),
$$

в которой старший коэффициент пропорционален мере Гаусса прямоугольника $[0, \xi] \times[0, \eta]$.

Функция $C(\xi, \eta)$, как и левая часть равенства $(2.7)$, разрывна во всех точках, у которых хотя бы одна координата рациональна. Эта функция задается сингулярным рядом, т. е. рядом, состоящим из остаточных членов асимптотических формул (см. [37], [44], [46], [47]).

Равенство (1.11) также обобщается на случай статистик Гаусса-Кузьмина:

$$
\frac{1}{\varphi(P)} \sum_{a=1}^{P} l_{\xi, \eta}\left(\frac{a}{P}\right)=\frac{2 \log P}{\zeta(2)} \int_{0}^{\xi} \int_{0}^{\eta} \frac{d \alpha d \beta}{(1+\alpha \beta)^{2}}+\widetilde{C}(\xi, \eta)+O\left(P^{-1 / 6+\varepsilon}\right)
$$

(при $\eta=1$ доказательство приведено в [43]; формула для главного члена следует из результата Хейльбронна $[25]$; функции $C(\xi, \eta)$ и $\widetilde{C}(\xi, \eta)$ выражаются друг через друга). Сравнивая равенство (2.2) с (2.7) (или с (2.8)), можно сделать вывод, что главные члены в непрерывных и дискретных задачах пропорциональны и определяются инвариантной мерой, а следующие значащие члены отличаются и имеют принципиально разную природу.

Аналитический аппарат, используемый при изучении статистических свойств конечных цепных дробей, позволяет решать и другие задачи, в которых цепные дроби используются в качестве вспомогательного инструмента.

В частности, это позволяет

- исследовать типичное поведение алгоритмов Евклида с округлением до ближайшего целого [48], [49], с четными и нечетными неполными частными 
[50]-[52], при делении с избытком [53], [54], при делении вычитанием [55], [56] и типичное поведение диагональных дробей Минковского [57] и цепных дробей более общего вида [58], а также изучать разложение в цепные дроби квадратичных рациональностей [45];

- в бильярде Синая (круглые отражатели находятся в узлах решетки $\mathbb{Z}^{2}$ ) находить плотность распределения случайной величины, равной длине свободного пробега частицы [59]-[62]; (в эквивалентной задаче) вычислять плотность совместного распределения длин соседних отрезков, соединяющих начало координат с примитивными точками целочисленной решетки [63], [64];

- описывать предельное распределение чисел Фробениуса с тремя аргументами [65]-[69];

- доказать существование предельной плотности распределения для неполных сумм Гаусса и неполных тета-рядов [70]-[72], а также находить эти плотности [73].

Необходимо отметить, что ко всем перечисленным задачам существуют другие подходы, основанные на эргодической теории и методах геометрии чисел. Обычно эргодические методы оказываются применимыми в более общей ситуации, но, по сравнению с аналитическими методами, требуют усреднения по большему числу параметров и приводят к менее точным остаточным членам.

В частности, с помощью эргодической теории удается

- проанализировать широкий класс алгоритмов Евклида [74]--[76];

- исследовать бильярд Синая в пространствах произвольной размерности [77], [78], изучить поведение свободного пробега частиц в квазикристаллических структурах [79];

- доказать существование предельной плотности распределения чисел Фробениуса с произвольным числом аргументов [80]; описать свойства этой плотности [81]--[83];

- получать результаты о поведении неполных сумм Гаусса и неполных тета-рядов [84], [85].

2.3. Вполне примитивные решетки. Через $M\left(v_{1}, \ldots, v_{s}\right)$ будем обозначать матрицу, в которой координаты векторов $v_{1}, \ldots, v_{s}$ записаны по столбцам. Полную решетку $\Lambda \subset \mathbb{Z}^{s}$ с базисом $\left(v_{1}, \ldots, v_{s}\right)$ будем называть вполне примитивной, если для каждой строки матрицы $M\left(v_{1}, \ldots, v_{s}\right)$ миноры, соответствующие элементам этой строки, взаимно просты в совокупности. Например, для матрицы $\left(\begin{array}{ll}a_{1} & a_{2} \\ b_{1} & b_{2}\end{array}\right)$ это условие означает, что $\left(a_{1}, a_{2}\right)=\left(b_{1}, b_{2}\right)=1$, т. е. при $s=2$ понятия примитивной и вполне примитивной решетки совпадают. Если $s=3$, то для базисной матрицы вида (1.8) условие вполне примитивности записывается в виде

$$
\begin{gathered}
\left(\left|\begin{array}{ll}
a_{1} & a_{2} \\
b_{1} & b_{2}
\end{array}\right|,\left|\begin{array}{ll}
a_{2} & a_{3} \\
b_{2} & b_{3}
\end{array}\right|,\left|\begin{array}{ll}
a_{1} & a_{3} \\
b_{1} & b_{3}
\end{array}\right|\right)=1, \\
\left(\left|\begin{array}{ll}
a_{1} & a_{2} \\
c_{1} & c_{2}
\end{array}\right|,\left|\begin{array}{ll}
a_{1} & a_{3} \\
c_{1} & c_{3}
\end{array}\right|,\left|\begin{array}{ll}
a_{2} & a_{3} \\
c_{2} & c_{3}
\end{array}\right|\right)=1, \\
\left(\left|\begin{array}{ll}
b_{1} & b_{2} \\
c_{1} & c_{2}
\end{array}\right|,\left|\begin{array}{ll}
b_{1} & b_{3} \\
c_{1} & c_{3}
\end{array}\right|,\left|\begin{array}{ll}
b_{2} & b_{3} \\
c_{2} & c_{3}
\end{array}\right|\right)=1 .
\end{gathered}
$$


Эквивалентное определение вполне примитивной решетки получается, если потребовать, чтобы решетка имела базис с матрицей

$$
\left(\begin{array}{lll}
1 & 0 & 0 \\
0 & 1 & 0 \\
a & b & P
\end{array}\right)
$$

где $0 \leqslant a, b<P$ и $(a, P)=(b, P)=1$. Отсюда, в частности, следует, что существует $\varphi^{2}(P)$ трехмерных вполне примитивных решеток с определителем $P$.

С точки зрения теории диофантовых приближений локальные минимумы решетки с базисной матрицей (2.12) являются наилучшими приближениями линейной формы $x_{1} \frac{a}{P}+x_{2} \frac{b}{P}+x_{3}$. Все сказанное выше о примитивных трехмерных решетках очевидным образом переносится на случай произвольной размерности $s \geqslant 3$.

2.4. Многомерный аналог меры Гаусса. Мера Гаусса является частным случаем более общей конструкции. Множество базисов в $s$-мерных решетках можно отождествить с множеством матриц $G L_{s}(\mathbb{R})$. Определения локальных минимумов и минимальных систем векторов (см. п. 3.1) не зависят от выбора масштабов на осях координат, поэтому для изучения свойств минимальных базисов естественно рассмотреть факторпространство $\mathscr{X}_{s}=D_{s}(\mathbb{R}) \backslash G L_{s}(\mathbb{R})$, где $D_{s}(\mathbb{R})$ - группа диагональных обратимых матриц размера $s \times s$ с действительными коэффициентами. Биинвариантная мера Хаара на $G L_{s}(\mathbb{R})$

$$
d \lambda(g)=\frac{d g}{(\operatorname{det} g)^{s}},
$$

где $g \in G L_{s}(\mathbb{R})$ и $d g=d g_{11} d g_{12} \cdots d g_{s s}$ - мера Лебега, индуцирует на $\mathscr{X}_{s}$ фактормеру $\mu$, являющуюся правой мерой Хаара и сохраняющую инвариантность относительно левого действия $D_{s}(\mathbb{R})$ : для любых $h \in D_{s}(\mathbb{R})$ и $g \in G=G L_{s}(\mathbb{R})$

$$
d \mu(h G)=d \mu(h G g) .
$$

Полагая $\bar{g}=\left(\bar{g}_{i j}\right) \in \mathscr{X}_{s}$, в карте $\bar{g}_{11}=\bar{g}_{22}=\cdots=\bar{g}_{s s}=1\left(\bar{g}_{i j}=g_{i j} g_{i i}^{-1}, 1 \leqslant\right.$ $i, j \leqslant s)$ меру $\mu$ можно задать равенством

$$
d \mu(\bar{g})=\frac{d \bar{g}}{(\operatorname{det} \bar{g})^{s}}, \quad \text { где } \quad d \bar{g}=d \bar{g}_{12} \cdots d \bar{g}_{s s-1} .
$$

При этом правая инвариантность $\mu$ будет следовать из формулы

$$
\frac{d g}{(\operatorname{det} g)^{s}}=\frac{d g_{11}}{g_{11}} \frac{d g_{22}}{g_{22}} \cdots \frac{d g_{s s}}{g_{s s}} \frac{d \bar{g}_{12} \cdots d \bar{g}_{s s-1}}{(\operatorname{det} \bar{g})^{s}},
$$

в которой $\frac{d g_{11}}{g_{11}} \frac{d g_{22}}{g_{22}} \cdots \frac{d g_{s s}}{g_{s s}}-$ мера Хаара на $D_{s}(\mathbb{R})$.

При $s=2$ мера $\mu$, взятая на пространстве матриц вида $\left(\begin{array}{cc}1 & -\alpha \\ \beta & 1\end{array}\right)$ (нормированных матриц Вороного), с точностью до нормировочного множителя $1 / \log 2$ 
совпадает с расширенной мерой Гаусса (2.4). При $s=3$ мера $\mu$ возникает при исследовании статистических свойств многогранников Клейна (см. [86], [87]) и цепных дробей Вороного-Минковского (см. [27]). Существенное различие между этими двумя объектами проявляется в том, что многогранники Клейна параметризуются точками всего пространства $\mathscr{X}_{s}$, мера которого бесконечна, а невырожденным минимальным системам векторов (т. е. системам, матрицы которых имеют ненулевой определитель, см. раздел 3$)$ в пространстве $\mathscr{X}_{s}$ соответствует область конечной меры $\mu$ : если матрица $g \in G L_{s}(\mathbb{R})$ с диагональным преобладанием (в каждой строке внедиагональные элементы по модулю не превосходят диагональных) задает невырожденную минимальную систему векторов решетки $\Lambda$, то, по теореме Минковского о выпуклом теле, $g_{11} g_{22} \cdots g_{s s} \leqslant \operatorname{det} \Lambda$, и

$$
|\operatorname{det} \bar{g}|=\frac{|\operatorname{det} g|}{g_{11} g_{22} \cdots g_{s s}} \geqslant \frac{|\operatorname{det} g|}{\operatorname{det} \Lambda} \geqslant 1 .
$$

\section{3. Цепные дроби и решетки}

3.1. Геометрия цепных дробей. Существуют две геометрические интерпретации классических цепных дробей, допускающие естественное обобщение на многомерный случай. В первой из них, принадлежащей Клейну (см. [88], [89], а также более раннее замечание Смита [90; с. 146-147]), цепная дробь идентифицируется с выпуклой оболочкой (многоугольником Клейна) точек целочисленной решетки, лежащих в двух смежных углах. Вторая интерпретация, предложенная независимо Вороным и Минковским (см. [17], [18] и [19], [91], а также переизложение оригинальных результатов в [20], [21] и [22]), основана на использовании локальных минимумов решеток, минимальных систем и экстремальных параллелепипедов (см. определения ниже). В плоских решетках вершины многоугольников Клейна (после линейного преобразования, переводящего стороны данных углов в оси координат) можно отождествить с локальными минимумами по Вороному. Однако, начиная с размерности 3, геометрические конструкции Клейна и Вороного-Минковского становятся различными (см. [92], [93]).

Напомним необходимые определения, восходящие к Вороному и Минковскому. Будем называть решетку $\Lambda \subset \mathbb{R}^{s}$ неприводимой (или решеткой общего положения), если координатные гиперплоскости не содержат узлов этой решетки, отличных от начала координат; в противном случае решетка называется приводимой. Множество полных s-мерных решеток (т. е. решеток, размерность которых совпадает с размерностью пространства) будем обозначать через $\mathscr{L}_{s}(\mathbb{R})$, а его подмножество, состоящее из неприводимых решеток, - чеpe3 $\mathscr{L}_{s}^{*}(\mathbb{R})$.

Для непустого конечного множества $A \subset \mathbb{R}^{s}$ положим

$$
\begin{aligned}
|A|_{i}= & \max \left\{\left|x_{i}\right|: x=\left(x_{1}, \ldots, x_{s}\right) \in A\right\} \quad(i=1, \ldots, s), \\
& \operatorname{Box}(A)=\left(-|A|_{1},|A|_{1}\right) \times \cdots \times\left(-|A|_{s},|A|_{s}\right), \\
& \overline{\operatorname{Box}}(A)=\left[-|A|_{1},|A|_{1}\right] \times \cdots \times\left[-|A|_{s},|A|_{s}\right] .
\end{aligned}
$$


Другими словами, Вох $(A)$ - это наименьший параллелепипед, описанный около множества $A$ (будем рассматривать только параллелепипеды с центром в начале координат и гранями, параллельными координатным плоскостям).

Системой узлов порядка $r$ решетки $\Lambda$ (не обязательно полной) назовем любой конечный набор $\left(v_{1}, \ldots, v_{r}\right)$ ненулевых узлов решетки, в котором $v_{i} \neq \pm v_{j}$ $(1 \leqslant i<j \leqslant r)$. Произвольной системе $S=\left(v_{1}, \ldots, v_{r}\right)$ будем ставить в соответствие матрицу $M\left(v_{1}, \ldots, v_{r}\right)$, записывая координаты векторов $v_{1}, \ldots, v_{r}$ по столбцам.

Узел $\gamma$ решетки $\Lambda \in \mathscr{L}_{s}(\mathbb{R})$ называется относительным (локальным) минимумом решетки $\Lambda$ по Вороному (в дальнейшем - просто минимумом), если параллелепипед $\overline{\mathrm{Box}}(\gamma)$ не содержит узлов решетки $\Lambda$, отличных от своих вершин и начала координат (см. [18]). Множество всех локальных минимумов решетки $\Lambda$ будем обозначать через $\mathfrak{M}(\Lambda)$. Если в решетке $\Lambda$ имеется несколько минимальных векторов $v_{1}, \ldots, v_{k}$ таких, что $\left|v_{i}\right|=\left|v_{j}\right|(1 \leqslant i<j \leqslant k)$, то договоримся включать в $\mathfrak{M}(S)$ лишь один из этих векторов.

Система $S$ векторов решетки $\Lambda$ называется минималъной, если параллелепипед $\operatorname{Box}(S)$ не содержит узлов решетки $\Lambda$, отличных от начала координат. В частности, для неприводимых решеток понятия "минимальная система порядка 1" и “локальный минимум" совпадают. Для приводимых решеток определение минимальной системы будет нуждаться в уточнении (см. [94]).

В двумерном случае на множестве локальных минимумов $\mathfrak{M}(\Lambda)$ введем структуру последовательности

$$
\mathfrak{M}(\Lambda)=\left(\ldots, v_{-2}, v_{-1}, v_{0}, v_{1}, v_{2}, \ldots\right),
$$

в которой векторы $v_{n}=\left((-1)^{n} x_{n}, y_{n}\right)\left(x_{n}, y_{n}>0\right)$ упорядочены по убыванию первой координаты: $y_{n+1}>y_{n}, x_{n+1}<x_{n}$. При этом каждая минимальная пара векторов имеет вид $\left(v_{n}, v_{n+1}\right)$ (т. е. состоит из соседних локальных минимумов) и является базисом решетки $\Lambda$ (см. [18]); такие пары будем называть базисами Вороного.

Рассматривая нормированные матрицы

$$
\left(\begin{array}{cc}
1 & \mp \alpha_{n} \\
\pm \beta_{n} & 1
\end{array}\right)=\left(\begin{array}{cc}
x_{n-1}^{-1} & 0 \\
0 & y_{n}^{-1}
\end{array}\right)\left(\begin{array}{cc}
x_{n-1} & \mp x_{n} \\
\pm y_{n-1} & y_{n}
\end{array}\right)
$$

получаем, что расширенное отображение Гаусса $\bar{T}\left(\alpha_{n}, \beta_{n}\right)=\left(\alpha_{n+1}, \beta_{n+1}\right)$ с геометрической точки зрения означает переход от базиса Вороного $\left(v_{n-1}, v_{n}\right)$ к смежному с ним базису $\left(v_{n}, v_{n+1}\right)$. Поэтому можно говорить, что расширенная мера Гаусса (2.4) описывает типичное поведение нормированных базисов Вороного.

Рациональному числу $r=a / P, 0 \leqslant a<P,(a, P)=1$, естественно поставить в соответствие решетку $\Lambda(r)$ с базисной матрицей $\left(\begin{array}{ll}1 & 0 \\ a & P\end{array}\right)$. Очевидно, что отображение $r \rightarrow \Lambda(r)$ устанавливает взаимно однозначное соответствие между дробями вида $a / P$, где $0 \leqslant a<P,(a, P)=1$, и двумерными примитивными решетками с определителем $P$. При $a \leqslant P / 2$ множество всех локальных минимумов решетки $\Lambda(a / P)$ совпадает с множеством вершин выпуклых оболочек точек решетки $\Lambda(r)$, лежащих в I и II координатных четвертях. При 
$a / P=\left[0 ; a_{1}, \ldots, a_{l}\right] \leqslant 1 / 2$ локальные минимумы имеют вид

$$
v_{0}=(-P, 0), \quad v_{1}=(a, 1), \quad \ldots, \quad v_{j}=\left((-1)^{j+1} x_{j}, y_{j}\right), \quad \ldots, \quad v_{l+1}=(0, P),
$$

где последовательности $\left\{x_{j}\right\}$ и $\left\{y_{j}\right\}$ определяются равенствами

$$
\frac{x_{j}}{x_{j-1}}=\left[0 ; a_{j}, \ldots, a_{l}\right], \quad \frac{y_{j-1}}{y_{j}}=\left[0 ; a_{j-1}, \ldots, a_{1}\right] \quad(1 \leqslant j \leqslant l+1) .
$$

(При $l=-1$ дробь $\left[0 ; a_{1}, \ldots, a_{l}\right]$ по определению считается равной $1 / 0$.) Множество базисов Вороного при этом совпадает с множеством пар $\left(v_{j}, v_{j+1}\right)$, где $0 \leqslant j \leqslant l$. При $a>P / 2$ координаты локальных минимумов определяются аналогичным образом по разложению в цепную дробь числа $(P-a) / P$.

3.2. Базисы Минковского. Пусть $\Pi=[0, \xi] \times[0, \eta] \subset[0,1]^{2}$. Обозначим через $N_{\Pi}(P)$ число базисов Вороного $\left(v_{n-1}, v_{n}\right)$ (во всех примитивных решетках с определителем $P$ ), для которых коэффициенты нормированной матрицы (3.2) удовлетворяет условию $\left(\alpha_{n}, \beta_{n}\right) \in \Pi$. Тогда равенство $(2.8)$ можно переписать в виде

$$
\frac{N_{\Pi}(P)}{\varphi(P)}=\frac{\bar{\nu}(\Pi)}{\zeta(2)} \log P+C(\Pi)+O\left(P^{-1 / 6+\varepsilon}\right),
$$

где

$$
\bar{\nu}(\Pi)=\frac{1}{\log 2} \int_{\Pi} \frac{d \alpha d \beta}{(1+\alpha \beta)^{2}}=\log _{2}(1+x y) .
$$

Обозначим через $G_{s}$ группу, действующую на множестве матриц размера $s \times s$, которая порождена следующими элементарными преобразованиями:

(i) перестановками столбцов и умножением столбцов на -1 (перенумерацией векторов базиса и изменением их ориентации);

(ii) перестановкой строк и умножением строк на -1 (переобозначением осей координат и изменением их направлений).

Две матрицы размера $s \times s$ будем считать эквивалентными, если они переводятся друг в друга действием группы $G_{s}$.

Как отмечалось выше, цель настоящей статьи - это развитие аналитических методов, позволяющих доказать трехмерный аналог равенства (2.8). В трехмерном случае классификация минимальных троек векторов несколько усложняется. Полное описание минимальных троек в решетках общего положения дает следующий результат Минковского.

Теорема 3.1 (Минковский). Пусть $S=\left(v_{1}, v_{2}, v_{3}\right)$ - минимальная система решетки $\Lambda \in \mathscr{L}_{3}^{*}$. Если система $S$ невырожденная, то она является базисом решетки $\Lambda$, а матрица $M\left(v_{1}, v_{2}, v_{3}\right)$ эквивалентна одной из двух канонических форм

$$
\begin{aligned}
& \left(\begin{array}{ccc}
x_{1} & x_{2} & -x_{3} \\
-y_{1} & y_{2} & y_{3} \\
z_{1} & -z_{2} & z_{3}
\end{array}\right), \text { где } x_{2} \geqslant x_{3}, \text { или } y_{3} \geqslant y_{1}, \text { или } z_{1} \geqslant z_{2}, \\
& \left(\begin{array}{ccc}
x_{1} & x_{2} & x_{3} \\
-y_{1} & y_{2} & y_{3} \\
z_{1} & -z_{2} & z_{3}
\end{array}\right), \text { где } y_{1}+y_{3} \geqslant y_{2} u\left(x_{2} \geqslant x_{3} \text { или } z_{2} \geqslant z_{1}\right) .
\end{aligned}
$$


Если же система $S$ вырожденная, то для некоторой комбинации знаков въполнено равенство $v_{1} \pm v_{2} \pm v_{3}=0$, а матрица $M\left(v_{1}, v_{2}, v_{3}\right)$ действием группљ $G_{3}$ приводится к виду

$$
\left(\begin{array}{ccc}
x_{1} & -x_{2} & -x_{3} \\
-y_{1} & y_{2} & -y_{3} \\
-z_{1} & -z_{2} & z_{3}
\end{array}\right), \quad \text { где } x_{2}+x_{3}=x_{1}, y_{1}+y_{3}=y_{2}, z_{1}+z_{2}=z_{3} .
$$

(Во всех трех случаях предполагается, что $x_{i}, y_{i}, z_{i} \geqslant 0$ и матрица базиса имеет диагональное преобладание: $x_{2}, x_{3} \leqslant x_{1}, y_{1}, y_{3} \leqslant y_{2}, z_{1}, z_{2} \leqslant z_{3}$.)

Верно и обратное: система из трех векторов $\left(v_{1}, v_{2}, v_{3}\right)$, матрица которой эквивалентна одной из матрич, вида (3.3), (3.4), является минимальной системой полной решетки $\Lambda=\left\langle v_{1}, v_{2}, v_{3}\right\rangle$; система векторов $\left(v_{1}, v_{2}, v_{3}\right)$ с матрицей вида (3.5) является минимальной системой решетки ранга 2: $\Lambda=\left\langle v_{1}, v_{2}, v_{3}\right\rangle=\left\langle v_{1}, v_{2}\right\rangle$.

Минковским эта теорема сформулирована без доказательства (см. [19], [91]). Подробное доказательство может быть найдено в [22; статьи 109-110] (см. также [95]-[98]).

В соответствии с теоремой 3.1 минимальные системы, матрицы которых эквивалентны матрицам (3.3), (3.4), будем называть базисами Минковского I и II типа соответственно.

Для приводимых решеток классификация минимальных систем усложняется (см. [94]). Однако основной интерес представляют именно базисы Минковского, поскольку любой локальный минимум $v$ можно дополнить до базиса Минковского, расширяя (в решетке общего положения, близкой к данной решетке $\Lambda)$ параллелепипед $\operatorname{Box}(v)$ вдоль осей координат.

3.3. Трехмерные цепные дроби. Для произвольного $T \subset \mathbb{R}^{3}$ положим $T^{\prime}=T$, если $0 \notin T$, и $T^{\prime}=T \backslash\{0\}$, если $0 \in T$;

$$
|T|=\left\{(|x|,|y|,|z|) \in \mathbb{R}^{3}:(x, y, z) \in T\right\} .
$$

Каждому дискретному множеству $T \subset \mathbb{R}^{3}(T \neq 0)$ поставим в соответствие ортогональную поверхность $\mathscr{P}(T)$, которая определяется как граница множества

$$
\left|T^{\prime}\right| \oplus \mathbb{R}_{\geqslant 0}^{3}=\left\{t+r: t \in\left|T^{\prime}\right|, r \in \mathbb{R}_{\geqslant 0}^{3}\right\} .
$$

Трехмерной иепной дробъю Вороного-Минковского, ассоциированной с решеткой $\Lambda$, будем называть ортогональную поверхность $\mathscr{P}(\Lambda)$.

Из дискретности $S$ следует, что поверхность $\mathscr{P}(S)$ внутри любого ограниченного множества имеет лишь конечное число вершин. Множество вогнутых вершин $\mathscr{P}(S)$ совпадает с $\mathfrak{M}(S)$ - множеством локальных минимумов. Каждой выпуклой вершине $\mathscr{P}(S)$ соответствует экстремальный параллелепипед параллелепипед вида $\operatorname{Box}\left(v_{1}, v_{2}, v_{3}\right)$, где $v_{1}, v_{2}, v_{3}$ - локальные минимумы, лежащие строго внутри трех взаимно перпендикулярных граней $\operatorname{Box}\left(v_{1}, v_{2}, v_{3}\right)$. Другими словами, экстремальный параллелепипед характеризуется тем, что его размеры нельзя увеличить так, чтобы он оставался свободным от точек множества $S$. 
Пример 3.2. Пусть $S$ состоит из ненулевых узлов решетки $\Lambda=\left\langle e_{1}, e_{2}, e_{3}\right\rangle$, где $e_{1}=(0,5,0), e_{2}=(0,0,5), e_{3}=(1,1,2)$. Тогда $\mathfrak{M}(S)=\left\{e_{1}, e_{2}, e_{3}, e_{4}, e_{5}\right\}$, где $e_{4}=(2,2,-1), e_{5}=(5,0,0)$. Поверхность $\mathscr{P}(S)$ изображена на рис. 1.

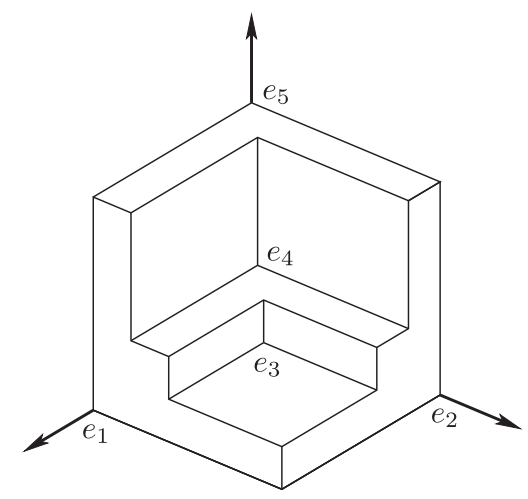

Рис. 1

Для описания полиэдра $\mathscr{P}(S)$ определим комплекс Минковского-Вороного $\mathrm{MV}(S)$ как двумерный комплекс, вершинами которого являются экстремальные параллелепипеды, ребрами - пары вида $\left(\operatorname{Box}\left(\gamma_{1}, \gamma_{2}, \gamma_{3}\right), \operatorname{Box}\left(\gamma_{2}, \gamma_{3}, \gamma_{4}\right)\right)$, а гранями - локальные минимумы $\gamma_{0}$, окруженные цепочками ребер вида

$$
\left(\operatorname{Box}\left(\gamma_{0}, \gamma_{1}, \gamma_{2}\right), \operatorname{Box}\left(\gamma_{0}, \gamma_{2}, \gamma_{3}\right)\right), \ldots,\left(\operatorname{Box}\left(\gamma_{0}, \gamma_{n}, \gamma_{1}\right), \operatorname{Box}\left(\gamma_{0}, \gamma_{1}, \gamma_{2}\right)\right)
$$

(см. рис. 2).

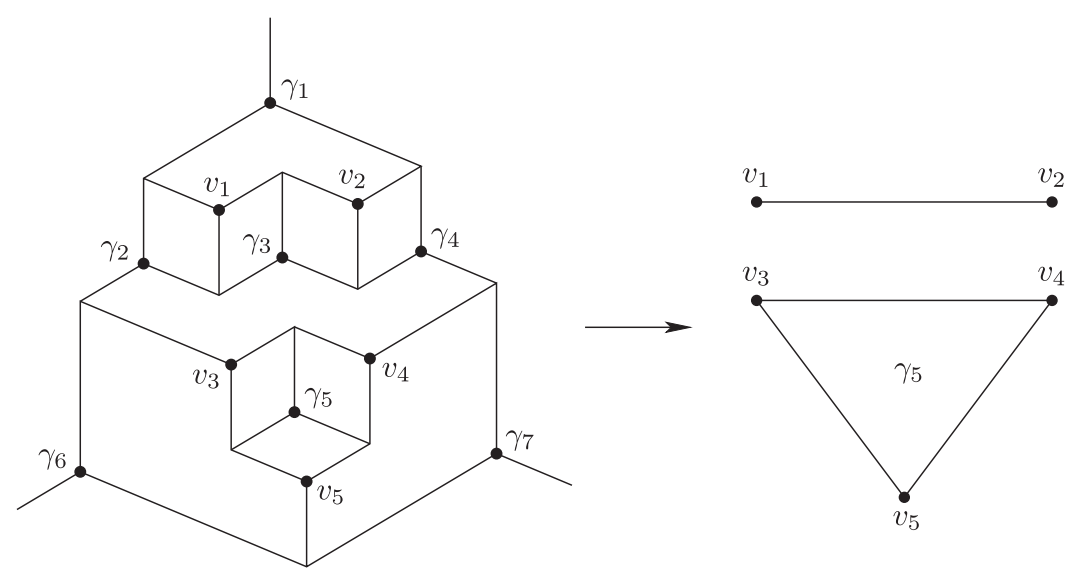

Рис. 2. Пример поверхности $\mathscr{P}(S)$ и соответствующего ей комплекса $\operatorname{MV}(S)$

Если $S$ - множество общего положения, то $\mathscr{P}(S)$ имеет более регулярную структуру. В этом случае естественно определить пару планарных взаимно двойственных графов - граф Вороного $\operatorname{Vor}(S)$ и граф Минковского $\operatorname{Min}(S)$. Bepшинами, ребрами и гранями графа Вороного (Минковского) будем считать соответственно вершины (грани), ребра и грани (вершины) комплекса $\operatorname{MV}(S)$. 

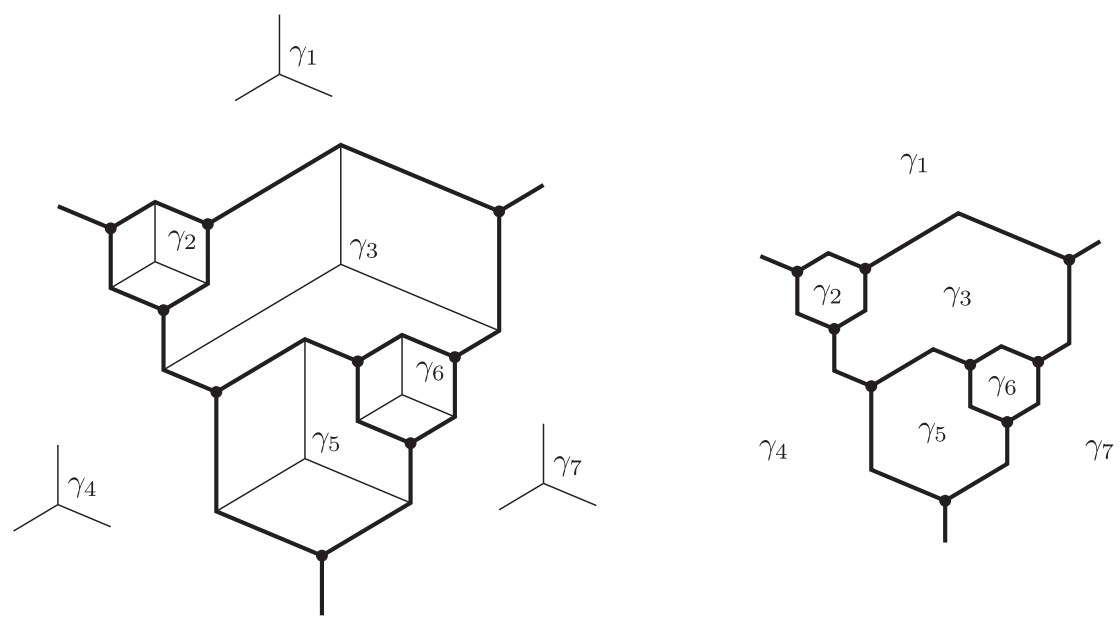

Рис. 3. Пример графа Вороного

Графы $\operatorname{Vor}(S)$ и $\operatorname{Min}(S)$ можно изобразить на поверхности $\mathscr{P}(S)$, используя следующие правила: вершины $\operatorname{Vor}(S)$ - это пики (выпуклые вершины) $\mathscr{P}(S)$, ребра - пары выпуклых ребер $\mathscr{P}(S)$ (все вершины $V(\Lambda)$ имеют степень 3 ), грани - области, образующиеся после стирания локальных минимумов и выходящих из них ребер (см. рис. 3); вершины $\operatorname{Min}(S)$ - это локальные минимумы (вогнутые вершины $\mathscr{P}(S)$ ), каждая грань - это треугольник, ребра которого соединяют три локальных минимума на поверхности некоторого экстремального параллелепипеда $(M(\Lambda)$ - триангуляция плоскости; вогнутые ребра $\mathscr{P}(S)$ можно считать частью ребер $\operatorname{Min}(S)$, см. рис. 4). Ребра каждого из графов
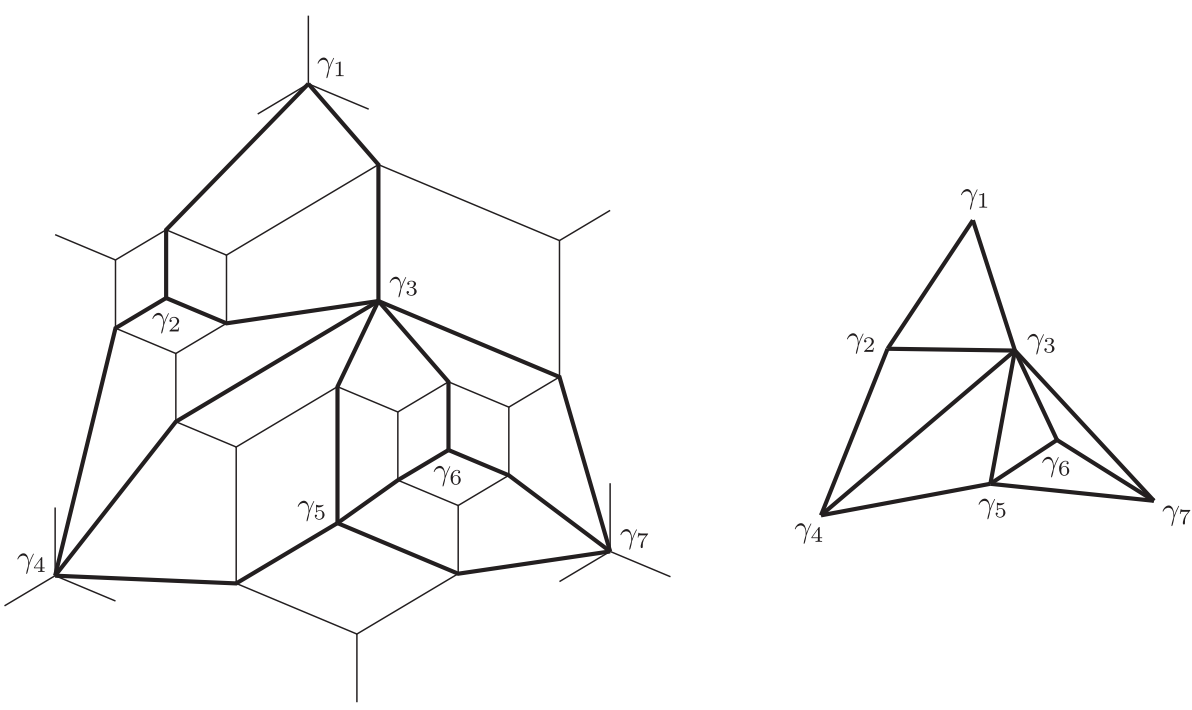

Рис. 4. Пример графа Минковского 

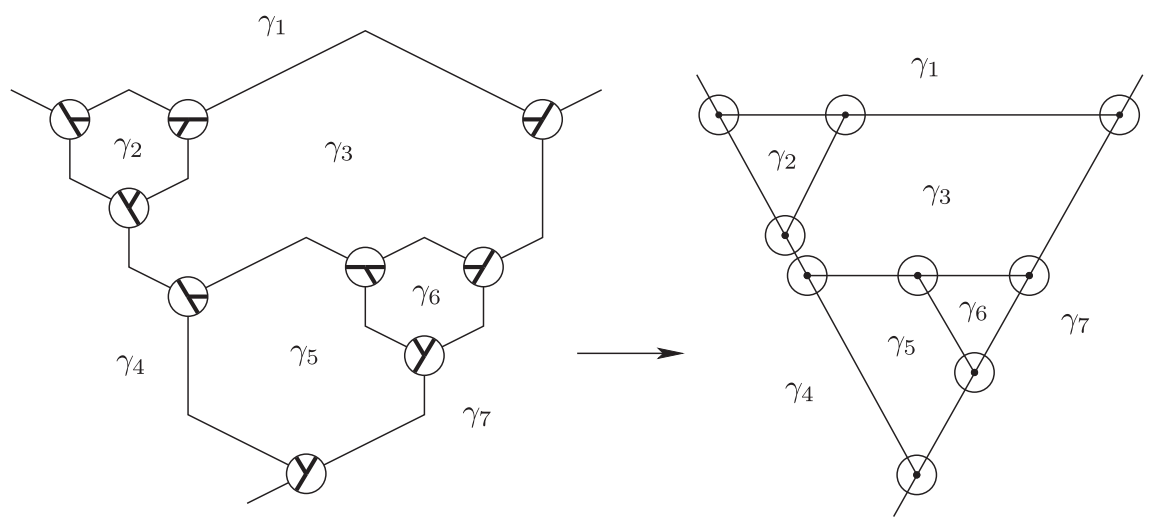

Рис. 5. Граф Вороного и его каноническая диаграмма

$\operatorname{Min}(S)$ и $\operatorname{Vor}(S)$ находятся во взаимно однозначном соответствии с седловыми вершинами $\mathscr{P}(S)$.

Граф Вороного удобно изображать на плоскости $x+y+z=0$ в виде канонической диаграммы - графа, ребрами которого являются отрезки трех направлений (см. рис. 5).

Каноническая диаграмма сохраняет информацию о взаимном расположении экстремальных параллелепипедов. Пусть $\gamma_{i}=\left( \pm x_{i}, \pm y_{i}, \pm z_{i}\right)(i=1,2,3)$ и матрица $M\left(\gamma_{1}, \gamma_{2}, \gamma_{3}\right)$ имеет диагональное преобладание. Предположим, что от экстремального параллелепипеда $\operatorname{Box}\left(\gamma_{1}, \gamma_{2}, \gamma_{3}\right)$ мы переходим к смежному с ним параллелепипеду, двигаясь по канонической диаграмме в "восточном" направлении (направление 1 на рис. 6). Тогда движение происходит по ребру с меткой $\left(x_{2}>x_{3}\right)$ и смежный сектор (см. рис. 6 справа) обозначен $\left(\gamma_{2}, \gamma_{3}\right)$. Это означает, что такой переход возможен, только если $x_{2}>x_{3}$, и смежный параллелепипед имеет вид $\operatorname{Box}\left(\gamma_{1}^{\prime}, \gamma_{2}, \gamma_{3}\right)$. Отсюда, в частности, следует, что существует 8 типов
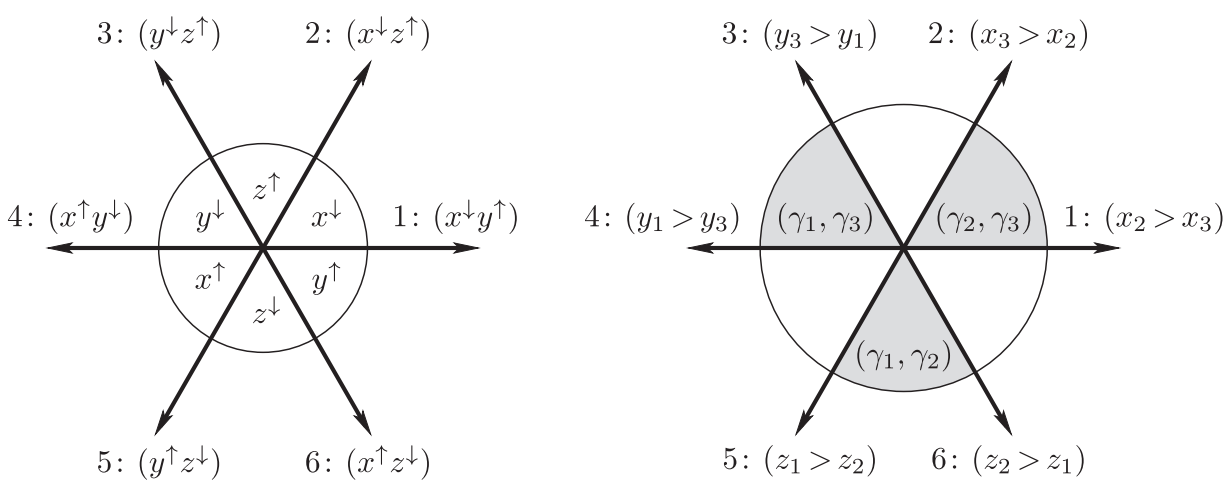

Рис. 6. Геометрический смысл направлений на канонической диаграмме 

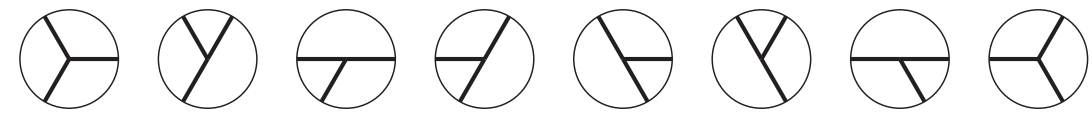

Рис. 7

локального строения вершин канонической диаграммы (из двух хорд, высекающих каждый из трех серых секторов, берется ровно одна) - см. рис. 7.

Смежные секторы на рис. 6 слева имеют метки $x^{\downarrow}$ и $y^{\uparrow}$, поэтому линейные размеры параллелепипеда $\operatorname{Box}\left(\gamma_{1}^{\prime}, \gamma_{2}, \gamma_{3}\right)$ по сравнению с $\operatorname{Box}\left(\gamma_{1}, \gamma_{2}, \gamma_{3}\right)$ меньше по первой координате, больше по второй и совпадают по третьей.

На графе Вороного, выбрав специальным образом ориентацию и раскраску ребер, можно ввести структуру деревъев Шнидера (см. [99]). Это позволяет изобразить любой конечный подграф графа $\operatorname{Vor}(S)$ в виде канонической диаграммы с сохранением взаимного расположения вершин (для любых двух выпуклых вершин поверхности $\mathscr{P}(S)$ их координаты в пространстве связаны теми же неравенствами, что и координаты соответствующих вершин канонической диаграммы на плоскости $x+y+z=0)$. Более подробная информация о графах Вороного и Минковского может быть найдена в [100].

Представляет интерес задача о нахождении необходимых и достаточных условий того, что граф, удовлетворяющий очевидным свойствам канонической диаграммы, действительно представляет собой каноническую диаграмму графа Вороного некоторой решетки. Неизвестно также, всегда ли бесконечный граф Вороного можно изобразить в виде канонической диаграммы с сохранением взаимного расположения вершин так, чтобы не возникало точек сгущения. Можно предположить, что это всегда возможно по крайней мере для периодических графов Вороного, соответствующих вполне вещественным кубическим полям.

Для данной решетки $\Lambda$ процесс построения трехмерной цепной дроби поверхности $\mathscr{P}(\Lambda)$ заключается в последовательном построении элементов $\mathscr{P}(\Lambda)$ - минимальных троек векторов, образующих грани $\operatorname{Min}(\Lambda)$. Некоторые тройки бывают вырожденными (см. теорему 3.1), однако такая ситуация не может возникать слишком часто: грани $\operatorname{Min}(\Lambda)$, соответствующие вырожденным тройкам, не могут быть смежными (см. [22; статья 11, теорема 3.3]). Для нахождения начального базиса Минковского применимы методы Вороного (см. [20; § 59]). На каждом следующем шаге по данному базису Минковского вычисляются смежные тройки. Если тройка оказывается вырожденной, то смежные с ней тройки уже являются базисами (формулы всех переходов выписываются явно см. [19], а также [22; с. 402-405]). Учитывая теорему 3.1, трехмерные цепные дроби можно понимать как динамическую систему с двумерным временем, инвариантной мерой (2.13) и фазовым пространством, состоящим из матриц множества $D_{3}(\mathbb{R}) \backslash G L_{3}(\mathbb{R})$, эквивалентных матрицам Минковского (3.3), (3.4). Алгоритм последовательного нахождения базисов Минковского можно применять и для целочисленных решеток, переходя при совпадении координат к бесконечно близким решеткам общего положения. 
Описанная выше конструкция трехмерных цепных дробей первоначально была предложена (в разных формах) Вороным и Минковским в качестве инструмента для нахождения фундаментальных единиц во вполне вещественных кубических полях. Кольцам целых чисел в таких полях соответствуют решетки $\Lambda$, для которых $\mathscr{P}(\Lambda)$ имеет двоякопериодическую структуру; таким образом, задача о нахождении фундаментальных единиц сводится к нахождению минимальных периодов $\mathscr{P}(\Lambda)$ (см. [20]). Например, пусть $\theta=2 \cos (2 \pi / 7), \theta^{\prime}=$ $2 \cos (4 \pi / 7), \theta^{\prime \prime}=2 \cos (6 \pi / 7)-$ корни кубического уравнения $\theta^{3}+\theta^{2}-2 \theta+1=0$. В качестве базиса кольца целых чисел поля $\mathbb{Q}(\theta)$ можно выбрать тройку $\left\{1, \theta, \theta^{2}\right\}$. Тогда фундаментальным единицам $\theta,-1-\theta$ соответствуют 2 независимых периода $\mathscr{P}(\Lambda)$, где $\Lambda$ - алгебраическая решетка, порожденная векторами $e_{j}=$ $\left(\theta^{j},\left(\theta^{\prime}\right)^{j},\left(\theta^{\prime \prime}\right)^{j}\right)(j=0,1,2)$, см. рис. 8 (жирными точками на рисунке обозначены вырожденные минимальные тройки векторов).

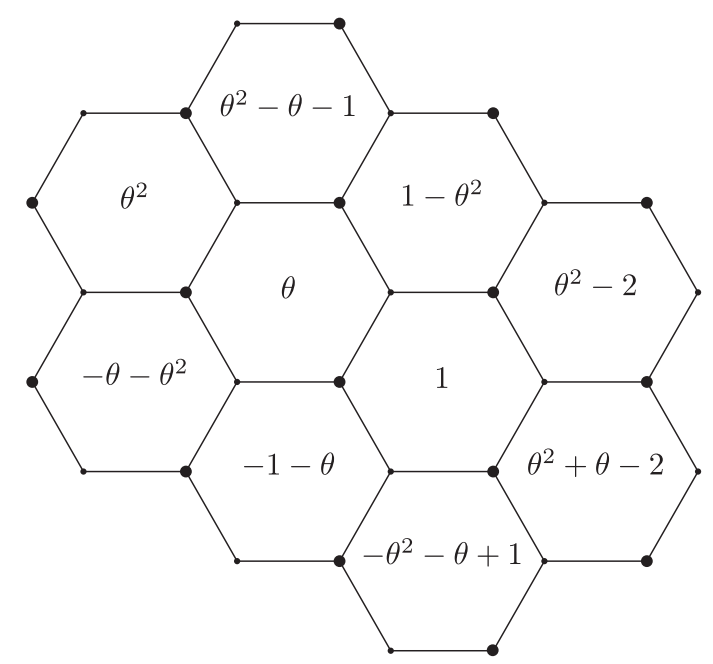

Рис. 8. Граф Вороного для поля $\mathbb{Q}(\theta), \theta=2 \cos (2 \pi / 7)$

На рис. 9 изображена каноническая диаграмма, построенная по тройке чисел $\theta=2 \cos (2 \pi / 9), \theta^{\prime}=2 \cos (4 \pi / 9), \theta^{\prime \prime}=2 \cos (6 \pi / 9)$, являющихся корнями кубического уравнения $\theta^{3}-3 \theta+1=0$. Базис кольца целых чисел поля $-\left\{1, \theta, \theta^{2}\right\}$, фундаментальные единицы - $\theta, 1-\theta$.

Два рассмотренных выше примера интересны тем, что числа $2 \cos (2 \pi / 7)$ и $2 \cos (2 \pi / 9)$ представляют собой начало трехмерного аналога спектра Маркова (см. [101], [102], а также теоремы изоляции в [103], [104]), в классической теории цепных дробей им соответствуют числа

$$
\frac{1+\sqrt{5}}{2}=2 \cos \frac{2 \pi}{5} \quad \text { и } \quad \sqrt{2}=2 \cos \frac{2 \pi}{8} .
$$

Дальнейшее развитие алгоритмов Вороного и Минковского см. в [105]-[112]. 


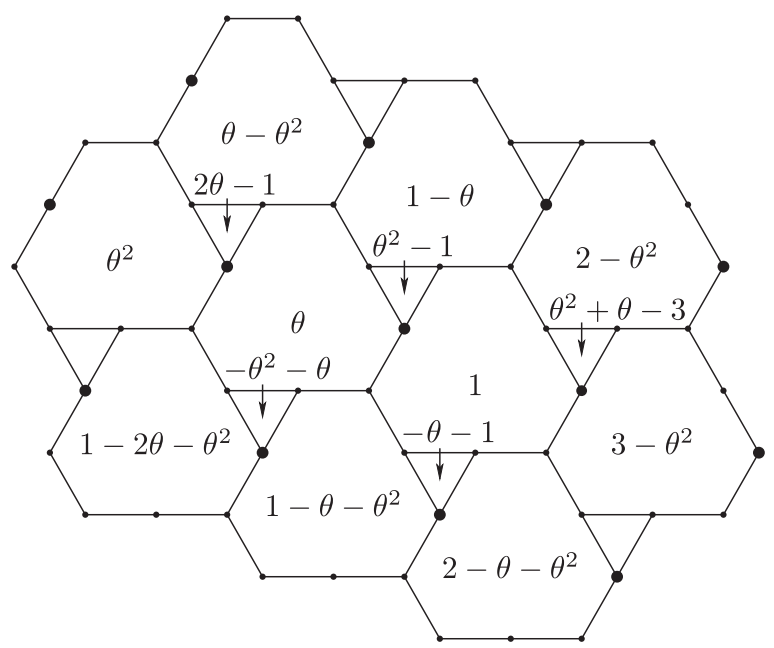

Рис. 9. Граф Вороного для поля $\mathbb{Q}(\theta), \theta=2 \cos (2 \pi / 9)$

Отметим также, что для трехмерных цепных дробей Вороного-Минковского удается доказать аналог теоремы Валена (см. [94], [95], [113]-[115]). О применении теории локальных минимумов см. [29], [30], [116]-[122].

Информация о других многомерных обобщениях цепных дробей может быть найдена в [123].

3.4. Формулировка основного результата. Трехмерным аналогом задачи о статистиках Гаусса для конечных цепных дробей является вопрос о статистических свойствах базисов Минковского, описанных в теореме 3.1. Вопрос о поведении в среднем элементов многогранников Клейна сводится к подсчету матриц Минковского с некоторыми дополнительными ограничениями или к подсчету матриц со сходными свойствами (см. [27]-[30]). Мы ограничимся рассмотрением базисов Минковского на вполне примитивных решетках (см. определение в п. 2.3), поскольку это приводит к более простому и естественному ответу.

Для матрицы $X$ вида (1.8) через $a, b$ и $c$ будем обозначать максимальные модули элементов в строках матрицы $X$ :

$$
a=\max \left\{\left|a_{1}\right|,\left|a_{2}\right|,\left|a_{3}\right|\right\}, \quad b=\max \left\{\left|b_{1}\right|,\left|b_{2}\right|,\left|b_{3}\right|\right\}, \quad c=\max \left\{\left|c_{1}\right|,\left|c_{2}\right|,\left|c_{3}\right|\right\} .
$$

Через $X^{\prime}, X^{\prime \prime}, X^{\prime \prime \prime}$ будем обозначать следующие матрицы:

$$
\begin{gathered}
X^{\prime}=\left(\begin{array}{ccc}
1 & 0 & 0 \\
0 & 1 & 0 \\
0 & 0 & c^{-1}
\end{array}\right) X, \quad X^{\prime \prime}=\left(\begin{array}{ccc}
1 & 0 & 0 \\
0 & b^{-1} & 0 \\
0 & 0 & c^{-1}
\end{array}\right) X \\
X^{\prime \prime \prime}=\left(\begin{array}{ccc}
a^{-1} & 0 & 0 \\
0 & b^{-1} & 0 \\
0 & 0 & c^{-1}
\end{array}\right) X .
\end{gathered}
$$


В частности, если $X$ - базисная матрица вида (3.3), (3.4), то матрицы $X^{\prime}$, $X^{\prime \prime}, X^{\prime \prime \prime}$ будут иметь соответственно вид

$$
\left(\begin{array}{ccc}
a_{1} & a_{2} & a_{3} \\
b_{1} & b_{2} & b_{3} \\
\gamma_{1} & \gamma_{2} & 1
\end{array}\right), \quad\left(\begin{array}{ccc}
a_{1} & a_{2} & a_{3} \\
\beta_{1} & 1 & \beta_{3} \\
\gamma_{1} & \gamma_{2} & 1
\end{array}\right), \quad\left(\begin{array}{ccc}
1 & \alpha_{2} & \alpha_{3} \\
\beta_{1} & 1 & \beta_{3} \\
\gamma_{1} & \gamma_{2} & 1
\end{array}\right),
$$

где $\left|\alpha_{i}\right|,\left|\beta_{i}\right|,\left|\gamma_{i}\right| \leqslant 1$.

Для произвольного матричного множества $M$ через $M(P), M^{\prime}, M^{\prime \prime}$ и $M^{\prime \prime \prime}$ будем обозначать следующие множества:

$$
\begin{aligned}
M(P) & =\{X \in M: \operatorname{det} X=P\}, & & M^{\prime \prime}=\left\{X^{\prime \prime}: X \in M\right\}, \\
M^{\prime} & =\left\{X^{\prime}: X \in M\right\}, & M^{\prime \prime \prime} & =\left\{X^{\prime \prime \prime}: X \in M\right\} .
\end{aligned}
$$

Пусть $\mathscr{M}$ - множество базисных матриц Минковского фиксированного типа (I или II) с фиксированной сигнатурой и целыми коэффициентами. Из теоремы 3.1 следует, что при подсчете матриц в множестве $\mathscr{M}$ достаточно ограничиться рассмотрением случаев, когда

$$
X=\left(\begin{array}{ccc}
a_{1} & a_{2} & a_{3} \\
b_{1} & b_{2} & b_{3} \\
c_{1} & c_{2} & c_{3}
\end{array}\right)=\left(\begin{array}{ccc}
x_{1} & x_{2} & \pm x_{3} \\
-y_{1} & y_{2} & y_{3} \\
z_{1} & -z_{2} & z_{3}
\end{array}\right)
$$

где $x_{i}=\left|a_{i}\right|, y_{i}=\left|b_{i}\right|, z_{i}=\left|c_{i}\right|$ удовлетворяют неравенствам теоремы 3.1.

Как отмечалось выше, основной результат статьи представляет собой трехмерное обобщение равенства (2.8) - асимптотической формулы для среднего значения статистик Гаусса-Кузьмина конечных цепных дробей. Определим трехмерные статистики Гаусса-Кузъмина следующим образом. Зафиксируем набор действительных чисел $\left(\xi_{2}, \xi_{3}, \eta_{1}, \eta_{3}, \zeta_{1}, \zeta_{2}\right) \in(0,1]^{6}$ и рассмотрим параллелепипед

$$
\Pi=\left[0, \xi_{2}\right] \times I\left(\xi_{3}\right) \times\left[-\eta_{1}, 0\right] \times\left[0, \eta_{3}\right] \times\left[0, \zeta_{1}\right] \times\left[-\zeta_{2}, 0\right],
$$

где $I\left(\xi_{3}\right)=\left[-\xi_{3}, 0\right]$ для матриц I типа и $I\left(\xi_{3}\right)=\left[0, \xi_{3}\right]$ для матриц II типа. Тогда трехмерными статистиками Гаусса-Кузьмина (соответствующими матрицам $\mathscr{M}$ данного типа и сигнатуры) для решетки $\Lambda \subset \mathbb{Z}^{3}$ будем называть суммы вида

$$
\sum_{X \in \mathscr{M}}[(\boldsymbol{\alpha}, \boldsymbol{\beta}, \boldsymbol{\gamma}) \in \Pi, X-\text { базисная матрица решетки } \Lambda],
$$

где $\boldsymbol{\alpha}=\left(\alpha_{2}, \alpha_{3}\right), \boldsymbol{\beta}=\left(\beta_{1}, \beta_{3}\right), \boldsymbol{\gamma}=\left(\gamma_{1}, \gamma_{2}\right)$, a $\alpha_{i}=\alpha_{i}(X), \beta_{i}=\beta_{i}(X), \gamma_{i}=\gamma_{i}(X)$ находятся из равенств (3.7), (3.8). При таком подходе трехмерным аналогом суммы, стоящей в левой части равенства (2.8), является величина

$$
\mathscr{N}_{\Pi}(P)=\sum_{X \in \mathscr{M}(P)}^{\#}[(\boldsymbol{\alpha}, \boldsymbol{\beta}, \boldsymbol{\gamma}) \in \Pi] .
$$

Здесь и ниже символ \# означает, что сумма берется по вполне примитивным матрицам $X$, т. е. по матрицам, удовлетворяющим условиям (2.9)-(2.11). 
Теорема 3.3. Для любого натурального $P$ и действительного $\varepsilon>0$

$$
\frac{\mathscr{N}_{\Pi}(P)}{\varphi^{2}(P)}=\mathscr{Q}_{2}(\log P)+O\left(P^{-1 / 34+\varepsilon}\right)
$$

где $\mathscr{Q}_{2}(x)$ - многочлен второй степени со стариим коэфбициентом

$$
\frac{\mu\left(\mathscr{M}^{\prime \prime \prime} \cap \Pi\right)}{2 \zeta(2) \zeta(3)}=\frac{1}{2 \zeta(2) \zeta(3)} \int_{\Pi} \frac{\left[X^{\prime \prime \prime} \in \mathscr{M}^{\prime \prime \prime}\right]}{\left(\operatorname{det} X^{\prime \prime \prime}\right)^{3}} d \boldsymbol{\alpha} d \boldsymbol{\beta} d \boldsymbol{\gamma}
$$

Подробная схема доказательства теоремы 3.3 будет дана в п. 5.3.

\section{4. Двумерный случай как модельная задача}

4.1. Постановка задачи. Будем записывать матрицы Вороного в виде

$$
A=\left(\begin{array}{ll}
a_{1} & a_{2} \\
b_{1} & b_{2}
\end{array}\right)=\left(\begin{array}{cc}
x_{1} & -x_{2} \\
y_{1} & y_{2}
\end{array}\right), \quad \text { где } \quad x_{1}, x_{2}, y_{1}, y_{2} \geqslant 0 .
$$

Через $\mathscr{V}$ обозначим множество всех примитивных матриц Вороного:

$$
\mathscr{V}=\left\{A=\left(\begin{array}{cc}
x_{1} & -x_{2} \\
y_{1} & y_{2}
\end{array}\right):\left(x_{1}, x_{2}\right)=\left(y_{1}, y_{2}\right)=1,0 \leqslant x_{2} \leqslant x_{1}, 0 \leqslant y_{1} \leqslant y_{2}\right\} .
$$

Через $a$ и $b$ (в пределах раздела 4) будем обозначать модули максимальных элементов в строках матрицы $A$ :

$$
a=\max \left\{\left|a_{1}\right|,\left|a_{2}\right|\right\}=x_{1}, \quad b=\max \left\{\left|b_{1}\right|,\left|b_{2}\right|\right\}=y_{2} .
$$

При этом $a b \leqslant P \leqslant 2 a b$. Для матрицы $A=\left(\begin{array}{ll}a_{1} & a_{2} \\ b_{1} & b_{2}\end{array}\right)$ через $A^{\prime}$ и $A^{\prime \prime}$ будем обозначать матрицы

$$
A^{\prime}=\left(\begin{array}{cc}
1 & 0 \\
0 & b^{-1}
\end{array}\right) A=\left(\begin{array}{cc}
x_{1} & -x_{2} \\
\beta & 1
\end{array}\right), \quad A^{\prime \prime}=\left(\begin{array}{cc}
a^{-1} & 0 \\
0 & b^{-1}
\end{array}\right) A=\left(\begin{array}{cc}
1 & -\alpha \\
\beta & 1
\end{array}\right) .
$$

Соответствующие множества будем обозначать через $\mathscr{V}^{\prime}$ и $\mathscr{V}^{\prime \prime}$ :

$$
\begin{aligned}
& \mathscr{V}^{\prime}=\left\{A^{\prime}=\left(\begin{array}{cc}
x_{1} & -x_{2} \\
\beta & 1
\end{array}\right):\left(x_{1}, x_{2}\right)=1,0 \leqslant x_{2} \leqslant x_{1}, \beta \in[0,1]\right\}, \\
& \mathscr{V}^{\prime \prime}=\left\{A^{\prime \prime}=\left(\begin{array}{cc}
1 & -\alpha \\
\beta & 1
\end{array}\right): \alpha, \beta \in[0,1]\right\} .
\end{aligned}
$$

Зафиксируем пару действительных чисел $\xi, \eta \in[0,1]$ и определим прямоугольник $\Pi=[0, \xi] \times[0, \eta]$. Рассмотрим задачу о подсчете величины

$$
N(P)=N_{\Pi}(P)=\left|\left\{A \in \mathscr{V}(P):\left(\frac{x_{2}}{x_{1}}, \frac{y_{1}}{y_{2}}\right) \in \Pi\right\}\right|,
$$

равной числу примитивных матриц Вороного с определителем $P$, для которых коэффициенты нормированной матрицы лежат в П. Ответом к этой задаче служит следующая теорема. 
Теорема 4.1. Пусть $P$ - натуралъное число, $\varepsilon>0$ - действителъное. Тогда

$$
\frac{N(P)}{\varphi(P)}=c_{1}(\Pi) \log P+c_{0}(\Pi)+O\left(P^{-1 / 8+\varepsilon}\right)
$$

¿əe

$$
c_{1}(\Pi)=\frac{\log (1+\xi \eta)}{\zeta(2)}=\frac{\bar{\nu}\left(\mathscr{V}^{\prime \prime} \cap \Pi\right)}{\zeta(2)}=\frac{1}{\zeta(2)} \int_{\Pi} \frac{\left[A^{\prime \prime} \in \mathscr{V}^{\prime \prime}\right] d \alpha d \beta}{\left(\operatorname{det} A^{\prime \prime}\right)^{2}}
$$

Остаточный член в теореме 4.1 хуже остаточного члена из результата Портера (1.11). Это обусловлено тем, что доказательство теоремы 4.1 проще: вместо оценок тригонометрических сумм по методу ван дер Корпута оно использует идею аппроксимации границ областей ступенчатыми функциями. Такой же подход будет лежать в основе доказательства теоремы 3.3 (трехмерного аналога теоремы 4.1). Ниже все основные шаги описаны кратко, чтобы наметить схему доказательства основного результата.

4.2. Разбиение на случаи. Множество всех матриц Вороного разобьем на две части:

$$
\mathscr{V}=\mathscr{V}_{1} \sqcup \mathscr{V}_{2}, \quad \mathscr{V}_{1}=\left\{A \in \mathscr{V}: x_{1} \leqslant y_{2}\right\}, \quad \mathscr{V}_{2}=\left\{A \in \mathscr{V}: x_{1}>y_{2}\right\}
$$

Величину $N(P)$, соответственно, можно представить в виде $N(P)=N_{1}(P)+$ $N_{2}(P)$, где определение $N_{\ell}(P)(\ell=1,2)$ получается из определения $N(P)$ наложением дополнительного условия $A \in \mathscr{V}_{\ell}$. Для доказательства теоремы 4.1 будет достаточно убедиться в справедливости асимптотической формулы

$$
\frac{N_{\ell}(P)}{\varphi(P)}=\frac{c_{1}(\Pi)}{2} \log P+c_{0}^{(\ell)}(\Pi)+O\left(P^{-1 / 8+\varepsilon}\right) \quad(\ell=1,2) .
$$

Из доказательства равенства (4.3) при $\ell=1$ будет следовать, что если в определении $N_{1}(P)$ нестрогое неравенство $x_{1} \leqslant y_{2}$ заменить на строгое неравенство $x_{1}>y_{2}$, то в формуле (4.3) изменится лишь вид константы $c_{0}^{(1)}(\Pi)$. Отображение

$$
\left(\begin{array}{cc}
x_{1} & -x_{2} \\
y_{1} & y_{2}
\end{array}\right) \rightarrow\left(\begin{array}{cc}
y_{2} & -y_{1} \\
x_{2} & x_{1}
\end{array}\right)
$$

устанавливает взаимно однозначное соответствие между матрицами из множества $\mathscr{V}_{1}$, для которых $x_{1}<y_{2}$, и матрицами из $\mathscr{V}_{2}$. Поэтому для доказательства равенства (4.3) при $\ell=2$ (а тем самым, и теоремы 4.1) достаточно убедиться в его справедливости при $\ell=1$. В дальнейшем будем предполагать, что $\ell=1$.

Через $\mathscr{V}_{\ell}(a, P)$ обозначим множество матриц $A \in \mathscr{V}_{\ell}(P)$, для которых $x_{1}=a$. Множества $\mathscr{V}_{\ell}^{\prime}(a, P)$ и $\mathscr{V}_{\ell}^{\prime \prime}(a, P)$ определяются по аналогии с $\mathscr{V}^{\prime}$ и $\mathscr{V}^{\prime \prime}$.

Чтобы проверить равенство (4.3), докажем сначала асимптотическую формулу для $N_{\ell}(a, P)=\left|\mathscr{V}_{\ell}(a, P)\right|$. Сделаем это двумя разными способами, первый раз - с помощью элементарных соображений, а второй раз - используя оценки сумм Клостермана. 
4.3. Линейная параметризация решений. Если зафиксированы числа $a_{1}$ и $a_{2}$, причем $\left(a_{1}, a_{2}\right)=1$, то можно подобрать целые $\widetilde{x}_{1}$ и $\widetilde{x}_{2}$ так, чтобы выполнялись равенства

$$
\left|\begin{array}{ll}
a_{1} & a_{2} \\
\widetilde{x}_{1} & \widetilde{x}_{2}
\end{array}\right|=1, \quad\left|\begin{array}{cc}
a_{1} & a_{2} \\
P \widetilde{x}_{1} & P \widetilde{x}_{2}
\end{array}\right|=P .
$$

Поэтому все решения уравнения

$$
\left|\begin{array}{ll}
a_{1} & a_{2} \\
b_{1} & b_{2}
\end{array}\right|=P
$$

относительно неизвестных $b_{1}, b_{2}$ допускают линейную параметризацию

$$
\left(\begin{array}{l}
b_{1} \\
b_{2}
\end{array}\right)=\left(\begin{array}{l}
\widetilde{b}_{1} \\
\widetilde{b}_{2}
\end{array}\right)+u\left(\begin{array}{l}
a_{1} \\
a_{2}
\end{array}\right)
$$

где $u \in \mathbb{Z}$ и $\widetilde{b}_{1,2}=P \widetilde{x}_{1,2}$. Из равенств

$$
\left(b_{1}, b_{2}\right)=\left(b_{1}, b_{2}, P\right)=\left(u a_{1}, u a_{2}, P\right)=(u, P)
$$

следует, что решение, полученное по формуле (4.5), будет задавать примитивную матрицу $A$ тогда и только тогда, когда $(u, P)=1$.

4.4. Первый вариант оценки остаточного члена. Получим асимптотическую формулу для $N_{\ell}(a, P)$ исходя из элементарных соображений. Из равенства $y_{2}=\left(P-x_{2} y_{1}\right) / x_{1}$ следует, что условия $y_{1} \leqslant y_{2}$ и $x_{1} \leqslant y_{2}$, характеризующие множество $\mathscr{V}_{\ell}(a, P)$ (напомним, что мы рассматриваем только случай $\ell=1)$, можно записать в виде $y_{1} \leqslant f\left(x_{2}\right)$, где

$$
f(t)=\min \left\{f_{1}(t), f_{2}(t)\right\}, \quad f_{1}(t)=\frac{P}{a+t}, \quad f_{2}(t)=\frac{P-a^{2}}{t} .
$$

При этом

$$
f(t) \leqslant f_{1}(t) \ll \frac{P}{a}, \quad\left|f_{1}^{\prime}(t)\right|=\frac{P}{(a+t)^{2}} \ll \frac{P}{a^{2}}
$$

и (при условии, что $\left.f(t)=f_{2}(t)\right)$

$$
\left|f_{2}^{\prime}(t)\right|=\frac{\left|f_{2}(t)\right|}{t} \ll \frac{P}{a t} .
$$

С помощью линейной параметризации (4.5) находим, что

$$
\sum_{0 \leqslant y_{1} \leqslant f\left(x_{2}\right)}^{\#} \delta_{a}\left(P-x_{2} y_{1}\right)=\frac{\varphi(P)}{a P} \int_{0}^{f\left(x_{2}\right)} d y_{1}+O\left(P^{\varepsilon}\right) .
$$

Переходя к переменной $\beta=y_{1} / y_{2}$ и пользуясь равенствами

$$
y_{1}=\frac{\beta P}{\operatorname{det} A^{\prime}}, \quad d y_{1}=\frac{a P d \beta}{\left(\operatorname{det} A^{\prime}\right)^{2}},
$$


перепишем формулу (4.9) в виде

$$
\sum_{y_{1}}\left[A \in \mathscr{V}_{\ell}(a, P)\right]=\varphi(P) \int_{\mathbb{R}} \frac{\left[A^{\prime} \in \mathscr{V}_{\ell}^{\prime}(a, P)\right] d \beta}{\left(\operatorname{det} A^{\prime}\right)^{2}}+O\left(P^{\varepsilon}\right) .
$$

Суммируя последнее равенство по $x_{2}$ и переходя к переменной $\alpha=x_{2} / x_{1}$, получаем

$$
\frac{N_{\ell}(a, P)}{\varphi(P)}=\frac{\varphi(a)}{a^{2}} \int_{\mathbb{R}^{2}} \frac{\left[A^{\prime \prime} \in \mathscr{V}_{\ell}^{\prime \prime}(a, P)\right] d \alpha d \beta}{\left(\operatorname{det} A^{\prime \prime}\right)^{2}}+\rho_{0}(a)+O\left(a P^{-1+\varepsilon}\right),
$$

где $\rho_{0}(a) \ll a^{-2+\varepsilon}$.

ЗАмечАниЕ 4.2. Обе части равенства (4.9) оцениваются как $O\left(P a^{-2}\right)$. Это позволяет, в частности, получить следующую асимптотическую формулу с тривиальной оценкой остатка:

$$
\begin{aligned}
& \sum_{Y_{1} \leqslant x_{2}<Y_{1}+Z_{1}} \sum_{0 \leqslant y_{1} \leqslant f\left(x_{2}\right)}^{\#} \delta_{a}\left(P-x_{2} y_{1}\right) \\
& =\frac{\varphi(P) \varphi(a)}{P a^{2}} \int_{Y_{1}}^{Y_{1}+Z_{1}} d x_{2} \int_{0}^{f\left(x_{2}\right)} d y_{1}+O\left(Z_{1} P^{1+\varepsilon} a^{-2}\right) .
\end{aligned}
$$

4.5. Второй вариант оценки остаточного члена. Второй подход к вычислению $N_{\ell}(a, P)$ заключается в подсчете числа решений сравнения $x y+P \equiv 0$ $(\bmod a)$, лежащих под графиком некоторой монотонной функции. Эту область будем аппроксимировать прямоугольниками, а в каждом прямоугольнике будем сводить задачу к оценкам сумм Клостермана.

ПРЕДЛОЖЕНИЕ 4.3. Пустъ $a>0 u$

$$
I=\left[Y_{1}, Y_{1}+Z_{1}\right) \times\left[Y_{2}, Y_{2}+Z_{2}\right) \subset[0, a) \times[0, P a) .
$$

Тогда справедлива асимптотическая формула

$$
\sum_{(x, y) \in I}^{\#} \delta_{a}(x y+P)=\frac{\varphi(P)}{P} \frac{\varphi(a)}{a^{2}} Z_{1} Z_{2}+O\left(R\left(Z_{2}\right)\right),
$$

¿əe

$$
R\left(Z_{2}\right) \ll\left(\frac{Z_{2}}{a}+a^{1 / 2}\right)(a, P)(a P)^{\varepsilon} .
$$

Предложение 4.3 доказывается стандартными методами (см., например, теорему 3 работы [24]; обобщение на случай произвольной линейной функции см. в [124]). Из формулы (4.12) следует, что для произвольной неотрицательной функции $f$ такой, что $Z_{2} \leqslant f(x) \leqslant Z_{2}+V\left(V \ll Z_{2}\right)$ при $x \in\left[Y_{1}, Y_{1}+Z_{2}\right)$, выполняется асимптотическая формула

$$
\begin{aligned}
& \sum_{Y_{1} \leqslant x_{2}<Y_{1}+Z_{2}} \sum_{0 \leqslant y_{1} \leqslant f\left(x_{2}\right)}^{\#} \delta_{a}\left(P-x_{2} y_{1}\right) \\
& =\frac{\varphi(P)}{P} \frac{\varphi(a)}{a^{2}} \int_{Y_{1}}^{Y_{1}+Z_{1}} d x \int_{0}^{f(x)} d y+O\left(\frac{Z_{1} V}{a}\right)+O\left(R\left(Z_{2}\right)\right) .
\end{aligned}
$$


Будем применять ее к функции $f$, определенной равенствами (4.6). Для этого выберем натуральное $r \leqslant a$ и представим отрезок $[0, a]$, внутри которого меняется переменная $x_{2}$, в виде

$$
[0, a]=\bigsqcup_{j=0}^{r-1} I(j)=\bigsqcup_{k=0}^{m} W_{k}
$$

где $m \ll \log a, I(0)=W_{0}=[0, a / r]$,

$$
I(j)=\left(\frac{j}{r} a, \frac{j+1}{r} a\right] \quad(1 \leqslant j<r), \quad W_{k}=\bigsqcup_{\substack{j=1 \\ 2^{k-1} \leqslant j<2^{k}}}^{r-1} I(j) \quad(k>0) .
$$

Величину $N_{\ell}(a, P)$ представим в виде

$$
N_{\ell}(a, P)=\sum_{k=0}^{m} N_{\ell, k}(a, P),
$$

где определение $N_{\ell, k}(a, P)$ получается из определения $N_{\ell}(a, P)$ наложением дополнительного условия $x_{2} \in W_{k}$. Аппроксимируя функцию $f$ на каждом интервале $I(j)$ постоянными функциями, можно доказать равенство

$$
N_{\ell, k}(a, P)=\frac{\varphi(P)}{P} \frac{\varphi(a)}{a^{2}} \int_{W_{k}} d x_{2} \int_{0}^{f\left(x_{2}\right)} d y_{1}+O\left(\frac{P}{a r}\right)+O\left(r R(b) P^{\varepsilon}\right) .
$$

Для этого при $k=0$ достаточно воспользоваться формулой (4.11), а при $k>0$ просуммировать равенство (4.13) по интервалам $I(j) \subset W_{k}$. На каждом из таких интервалов, согласно (4.7) и (4.8), $\left|f^{\prime}(x)\right| \ll r P \cdot 2^{-k} a^{-2}$. Значения функции $f$ меняются внутри отрезка длины $V=O\left(P \cdot 2^{-k} a^{-1}\right)$, что приводит к остатку $O\left(Z_{1} V / a\right)=O\left(P \cdot 2^{-k} a^{-1} r^{-1}\right)$. Остается учесть, что число интервалов $I(j) \subset W_{k}$ есть $O\left(2^{k}\right)$.

Просуммируем равенство (4.14) по $k$ и перейдем к переменным $\beta=y_{1} / y_{2}$ и $\alpha=x_{2} / x_{1}$ :

$$
\frac{N_{\ell}(a, P)}{\varphi(P)}=\frac{\varphi(a)}{a^{2}} \int_{\mathbb{R}^{2}} \frac{\left[A^{\prime \prime} \in \mathscr{V}_{\ell}^{\prime \prime}(a, P)\right] d \alpha d \beta}{\left(\operatorname{det} A^{\prime \prime}\right)^{2}}+O\left(\frac{P^{\varepsilon}}{r a}\right)+O\left(r R(b) P^{-1+\varepsilon}\right) .
$$

Значение $r=\left[P^{1 / 2} a^{-3 / 4}\right]$ выбирается исходя из соотношения $P /(r a) \asymp r a^{1 / 2}$. Требование $r \leqslant a$ будет выполнено при условии, что $a \geqslant P^{2 / 7}$. В результате получается асимптотическая формула для $N_{\ell}(a, P)$ со вторым вариантом остатка:

$$
\frac{N_{\ell}(a, P)}{\varphi(P)}=\frac{\varphi(a)}{a^{2}} \int_{\mathbb{R}^{2}} \frac{\left[A^{\prime \prime} \in \mathscr{V}_{\ell}^{\prime \prime}(a, P)\right] d \alpha d \beta}{\left(\operatorname{det} A^{\prime \prime}\right)^{2}}+O\left((a, P) a^{-1 / 4} P^{1 / 2+\varepsilon}\right) .
$$

4.6. Оценка итогового остатка. Сравнивая формулы (4.10) и (4.15), находим, что первую имеет смысл применять при $a \leqslant P^{2 / 5}$, а вторую - при $a>P^{2 / 5}$. Таким образом, для суммы всех остаточных членов получаем оценку

$$
\sum_{a \leqslant P^{2 / 5}} a P^{\varepsilon}+\sum_{P^{2 / 5}<a \leqslant P^{1 / 2}}(a, P) a^{-1 / 4} P^{1 / 2+\varepsilon} \ll P^{7 / 8+\varepsilon} .
$$


4.7. Подсчет главного члена. Если $a>\sqrt{q}$, то множество $\mathscr{V}_{\ell}(a, P)$ становится пустым. Несмотря на это, формуле (4.10) можно придать смысл, полагая $\rho_{0}(a)=0$. При такой договоренности из оценки $\rho_{0}(a) \ll a^{-2+\varepsilon}$ следует, что

$$
\sum_{a \leqslant P^{2 / 5}} \rho_{0}(a)=\sum_{a=1}^{\infty} \rho_{0}(a)+O\left(P^{-2 / 5+\varepsilon}\right) .
$$

Как отмечалось выше, условие $\alpha_{1} \leqslant \beta_{2}$, которому удовлетворяют матрицы множества $\mathscr{V}_{\ell}^{\prime \prime}(a, P)$, равносильно неравенству $a^{2} \leqslant P / \operatorname{det} A^{\prime \prime}$. Поэтому из соотношений $(4.10),(4.15)$ и (4.16) следует, что

$$
\frac{N_{\ell}(P)}{\varphi(P)}=\sum_{a=1}^{\infty} \frac{\varphi(a)}{a^{2}} \int_{\mathbb{R}^{2}} \frac{\left[A^{\prime \prime} \in \mathscr{V}_{\ell}^{\prime \prime}(a, P)\right] d \alpha d \beta}{\left(\operatorname{det} A^{\prime \prime}\right)^{2}}+\sum_{a=1}^{\infty} \rho_{0}(a)+O\left(P^{-1 / 8+\varepsilon}\right) .
$$

Условие $a \leqslant y_{2}$, которому должны удовлетворять матрицы множества $\mathscr{V}_{\ell}(a, P)$, не инвариантно относительно левого действия $D_{3}(\mathbb{R})$. При переходе к множеству $\mathscr{V}_{\ell}^{\prime \prime}(a, P)$ это условие принимает вид $a^{2} \leqslant P / \operatorname{det} A^{\prime \prime}$, т. е.

$$
\mathscr{V}_{\ell}^{\prime \prime}(a, P)=\left\{A^{\prime} \in \mathscr{V}_{\ell}^{\prime \prime}: a^{2} \leqslant \frac{P}{\operatorname{det} A^{\prime \prime}}\right\} .
$$

Поэтому

$$
\begin{aligned}
\frac{N_{\ell}(P)}{\varphi(P)}=\int_{\mathbb{R}^{2}} \frac{\left[A^{\prime \prime} \in \mathscr{V}_{\ell}^{\prime \prime}\right] d \alpha d \beta}{\left(\operatorname{det} A^{\prime \prime}\right)^{2}} \sum_{a=1}^{\infty} \frac{\varphi(a)}{a^{2}}\left[a^{2} \leqslant \frac{P}{\operatorname{det} A^{\prime \prime}}\right] \\
+\sum_{a=1}^{\infty} \rho_{0}(a)+O\left(P^{-1 / 8+\varepsilon}\right) .
\end{aligned}
$$

Применяя к внутренней сумме равенство

$$
\sum_{a=1}^{N} \frac{\varphi(a)}{a^{2}}=\frac{1}{\zeta(2)}\left(\log N+\gamma-\frac{\zeta^{\prime}(2)}{\zeta(2)}\right)+O\left(N^{-1+\varepsilon}\right)
$$

приходим к требуемой формуле (4.3).

\section{5. Разбиение на случаи}

В двумерном случае множество $\mathscr{V}$ разбивалось на два подмножества $\mathscr{V}_{1}$ и $\mathscr{V}_{2}$, почти не отличающихся друг от друга (см. п. 4.2). В трехмерном случае разбиение будет устроено более сложно и будет существенно использовать геометрические свойства базисов Минковского.

5.1. Приведенные матрицы. Пусть $X$ - базисная матрица Минковского вида (1.8) и $a, b, c$ определены равенствами (3.6). Тогда выполняется соотношение $P \asymp a b c$. Действительно, с одной стороны, имеет место очевидное неравенство $P \leqslant 6 a b c$. С другой стороны, по теореме Минковского о выпуклом теле 
$P \geqslant a b c$. В частности, неравенство $P \geqslant a b c$ означает, что среди миноров, соответствующих элементам любой строки $X$, всегда найдется по крайней мере один, имеющий максимально возможный порядок. Например,

$$
\max \left\{\left|a_{1} b_{2}-a_{2} b_{1}\right|,\left|a_{1} b_{3}-a_{3} b_{1}\right|,\left|a_{2} b_{3}-a_{3} b_{2}\right|\right\} \geqslant \frac{a b}{3} .
$$

Важную роль в дальнейших рассуждениях будет играть разбиение множества всех базисных матриц Минковского на карты - подмножества, на которых будет проводиться редукция Линника-Скубенко. В каждой карте положение максимального по порядку минора (для каждой строки) будет фиксировано.

ОПредЕЛЕНИЕ 5.1. Пусть $A=\left(\begin{array}{ll}a_{1} & a_{2} \\ b_{1} & b_{2}\end{array}\right), q=\operatorname{det} A \neq 0$, и $X-$ матрица базиса Минковского вида (1.8). Будем называть матрицу $X$ nриведенной, если она удовлетворяет следующим условиям.

$1^{\circ} . a_{1}=a$.

$2^{\circ} \cdot q \geqslant a b / 4$.

$3^{\circ}$. Базис $\left(e_{1}, e_{2}\right)$ решетки $\Lambda=\left\langle e_{1}, e_{2}\right\rangle$ близок к минимальному в следующем смысле: один из базисов $\left(e_{1}, e_{2}\right),\left(e_{1}, e_{1}+e_{2}\right),\left(e_{2}, e_{1} \pm e_{2}\right)$ является базисом Вороного решетки $\Lambda$.

Другими словами, свойства $1^{\circ}$ и $2^{\circ}$ означают, что в приведенной матрице угловой минор $q$ и угловой элемент $a_{1}$ имеют наибольшие возможные по порядку значения. Свойство $3^{\circ}$ означает, что и матрица $A$ по решетке $\Lambda$ с базисом $\left(a_{1}, b_{1}\right),\left(a_{2}, b_{2}\right)$ восстанавливается почти однозначно: число базисов Вороного в решетке с определителем $q$, так же как и длина разложения числа $d / q$ в цепную дробь, оценивается как $O(\log (q+1))$, поэтому и число возможных матриц $A$ при заданной решетке $\Lambda$ ограничено величиной $O(\log (q+1))$.

Будем обозначать через $g_{1}, \ldots, g_{6}$ элементы группы $G_{3}$, переставляющие местами коэффициенты $a, b, c$ матрицы $X$ (и сохраняющие диагональное преобладание $X)$.

Лемма 5.2. Множество $\mathscr{M}$ можно разбить на конечное число подмножеств так, что

(i) каждое множество разбиения задается конечным набором неравенств, инвариантных относительно левого действия $D_{3}(\mathbb{R})$;

(ii) если $\widetilde{\mathscr{M}}$ - одно из множеств разбиения, то для любого $i=1, \ldots, 6$ по крайней мере одно из множеств $g_{i}(\widetilde{\mathscr{M}})$ или $g_{i}(\widetilde{\mathscr{M}})\left(\begin{array}{lll}1 & 0 & 0 \\ 0 & 0 & 1 \\ 0 & 1 & 0\end{array}\right)$ coстоит из приведенных матрии.

ДокАзАтЕльство. Согласно теореме 3.1, необходимое разбиение достаточно построить для множеств (3.3) и (3.4). В каждом из них добавим дополнительное разбиение всеми гиперплоскостями вида $x_{i}=x_{j}, y_{i}=y_{j}, z_{i}=z_{j}(i \neq j)$. Рассмотрим произвольное множество из полученного разбиения. Если оно состоит из матриц I типа, то все его элементы удовлетворяют условиям $1^{\circ}-3^{\circ}$ (матрица $A=\left(\begin{array}{ll}a_{1} & a_{2} \\ b_{1} & b_{2}\end{array}\right)$ задает базис Вороного, поскольку $b_{1} \leqslant 0$ ). 
Пусть рассматриваемое множество состоит из матриц II типа. После того как строки матрицы $X$ упорядочены по возрастанию максимальных элементов, возникает три варианта расстановки знаков. Кроме случая (3.4) возможны также следующие два:

$$
\begin{aligned}
& \left(\begin{array}{ccc}
x_{1} & x_{2} & -x_{3} \\
-y_{1} & y_{2} & y_{3} \\
z_{1} & z_{2} & z_{3}
\end{array}\right), \quad \text { где } x_{2}+x_{3} \geqslant x_{1} \text { и }\left(y_{1} \geqslant y_{3} \text { или } z_{1} \geqslant z_{2}\right), \\
& \left(\begin{array}{ccc}
x_{1} & x_{2} & -x_{3} \\
y_{1} & y_{2} & y_{3} \\
z_{1} & -z_{2} & z_{3}
\end{array}\right), \quad \text { где } z_{1}+z_{2} \geqslant z_{3} \text { и }\left(x_{3} \geqslant x_{2} \text { или } y_{3} \geqslant y_{1}\right) .
\end{aligned}
$$

В матрицах (3.4) и (5.1) элемент $b_{1}$ отрицателен. Поэтому, как и для матриц I типа, $q=x_{1} y_{2}+x_{2} y_{1} \geqslant x_{1} y_{2}=a b$ и базис $\left(e_{1}, e_{2}\right)$ является базисом Вороного. Для матриц (5.2) дополнительно сделаем подразбиение плоскостями $a_{2}=a_{1} / 2$ и $b_{1}=b_{2} / 2$ и рассмотрим два случая:

1) $b_{1}>0$ и $\left(a_{2} \leqslant a_{1} / 2\right.$ или $\left.\left(a_{2}>a_{1} / 2, b_{1} \leqslant b_{2} / 2\right)\right)$;

2) $b_{1}>0, a_{2}>a_{1} / 2, b_{1}>b_{2} / 2$.

В первом случае $q=x_{1} y_{2}-x_{2} y_{1} \geqslant x_{1} y_{2} / 2=a b / 2$, и при $a_{2} \leqslant a_{1} / 2$ в качестве базиса Вороного можно выбрать пару $\left(e_{1}-e_{2}, e_{2}\right)$ с матрицей $\left(\begin{array}{ll}x_{1}-x_{2} & x_{2} \\ y_{1}-y_{2} & y_{2}\end{array}\right)$, а при $b_{1} \leqslant b_{2} / 2$ - пару $\left(e_{1}, e_{2}-e_{1}\right)$ с матрицей $\left(\begin{array}{cc}x_{1} & -\left(x_{1}-x_{2}\right) \\ y_{1} & y_{2}-y_{1}\end{array}\right)$.

Во втором случае в матрице $X$ переставим второй и третий столбцы:

$$
X=\left(\begin{array}{ccc}
x_{1} & x_{2} & -x_{3} \\
y_{1} & y_{2} & y_{3} \\
z_{1} & -z_{2} & z_{3}
\end{array}\right) \rightarrow\left(\begin{array}{ccc}
x_{1} & -x_{3} & x_{2} \\
y_{1} & y_{3} & y_{2} \\
z_{1} & z_{3} & -z_{2}
\end{array}\right) \sim\left(\begin{array}{ccc}
x_{1} & x_{3} & -x_{2} \\
-y_{1} & y_{3} & y_{2} \\
z_{1} & -z_{3} & z_{2}
\end{array}\right)
$$

Если в матрице $\left(\begin{array}{cc}x_{1} & x_{3} \\ -y_{1} & y_{3}\end{array}\right)$ будет выполняться условие $y_{3} \geqslant y_{1}$, то базис решетки $\Lambda=\left\langle e_{1}, e_{2}\right\rangle$, состоящий из векторов $e_{1}=\left(x_{1},-y_{1}\right), e_{2}=\left(x_{3}, y_{3}\right)$, будет базисом Вороного и $q=x_{1} y_{3}+x_{3} y_{1} \geqslant x_{1} y_{1} \geqslant a b / 2$. В оставшемся случае $\left(y_{3}<y_{1}, x_{3} \geqslant x_{2}\right)$ в качестве базиса Вороного можно выбрать пару $\left(e_{2}, e_{2}-e_{1}\right)$ с матрицей $\left(\begin{array}{cc}x_{3} & -\left(x_{1}-x_{3}\right) \\ y_{3} & y_{1}+y_{3}\end{array}\right)$. При этом $q=x_{1} y_{3}+x_{3} y_{1} \geqslant y_{1} x_{2} \geqslant a b / 4$. Лемма доказана.

Таким образом, каждой матрице $A$ можно поставить в соответствие базис Вороного решетки $\Lambda$, причем каждому базису Вороного будет соответствовать не более четырех матриц $A$.

ЗАмечание 5.3. Теорему 3.3 достаточно доказать, заменив в ее формулировке (и в определении (3.10) величины $\left.\mathscr{N}_{\Pi}(P)\right)$ множество $\mathscr{M}$ на множество $\widetilde{\mathscr{M}}$ - произвольное множество разбиения, построенного в лемме 5.2 . При этом множество $\widetilde{\mathscr{M}}$ можно представить в виде

$$
\widetilde{\mathscr{M}}=\bigsqcup_{\ell=1}^{6} g_{\ell}\left(\mathscr{M}_{\ell}\right)
$$


где $g_{\ell} \in G_{3}$ и каждое из множеств $\mathscr{M}_{\ell}$ состоит из приведенных матриц, удовлетворяющих условиям $a \leqslant b \leqslant c$ (в определении $\mathscr{M}_{\ell}$ нестрогие неравенства между $a, b$ и $c$ могут заменяться строгими, чтобы множества $g_{\ell}\left(\mathscr{M}_{\ell}\right)$ попарно не пересекались).

ЗАмечание 5.4. Трехмерная мера Гаусса инвариантна относительно левого действия $D_{3}(\mathbb{R})$. В частности, при $\beta_{1}^{\prime}=\beta_{1} / \beta_{3}, \beta_{2}^{\prime}=1 / \beta_{3}, \gamma_{1}^{\prime}=\gamma_{1} / \gamma_{2}, \gamma_{3}^{\prime}=1 / \gamma_{2}$ имеет место равенство

$$
\left|\begin{array}{ccc}
1 & \alpha_{2} & \alpha_{3} \\
\beta_{1} & 1 & \beta_{3} \\
\gamma_{1} & \gamma_{2} & 1
\end{array}\right|^{-3} \quad d \beta_{1} d \beta_{3} d \gamma_{1} d \gamma_{2}=\left|\begin{array}{ccc}
1 & \alpha_{2} & \alpha_{3} \\
\beta_{1}^{\prime} & \beta_{2}^{\prime} & 1 \\
\gamma_{1}^{\prime} & 1 & \gamma_{3}^{\prime}
\end{array}\right|^{-3} \quad d \beta_{1}^{\prime} d \beta_{2}^{\prime} d \gamma_{1}^{\prime} d \gamma_{3}^{\prime} .
$$

Значит, мера множества $D_{3}(\mathbb{R}) \backslash \mathscr{M}$ не зависит от того, были переставлены второй и третий столбцы матрицы $X$ или нет.

Из замечаний 5.3 и 5.4 вытекает, что для доказательства теоремы 3.3 достаточно убедиться в справедливости следующего утверждения.

Теорема 5.5. Пусть $\mathscr{M}_{\ell}$ - одно из множеств разбиения (5.3) и параллелепипед П определен равенством (3.9). Тогда

$$
\frac{1}{\varphi^{2}(P)} \sum_{X \in \mathscr{M}_{\ell}(P)}^{\#}[(\boldsymbol{\alpha}, \boldsymbol{\beta}, \boldsymbol{\gamma}) \in \Pi]=\mathscr{Q}_{2}^{(\ell)}(\log P)+O\left(P^{-1 / 34+\varepsilon}\right),
$$

где $\mathscr{Q}_{2}^{(\ell)}$ - многочлен второй степени со стариим коэфбициентом

$$
\frac{\mu\left(\widetilde{\mathscr{M}^{\prime \prime \prime}} \cap \Pi\right)}{12 \zeta(2) \zeta(3)}=\frac{1}{12 \zeta(2) \zeta(3)} \int_{\Pi} \frac{\left[X^{\prime \prime \prime} \in \widetilde{\mathscr{M}^{\prime \prime \prime}}\right]}{\left(\operatorname{det} X^{\prime \prime \prime}\right)^{3}} d \boldsymbol{\alpha} d \boldsymbol{\beta} d \boldsymbol{\gamma} .
$$

Для простоты изложения доказательство теоремы 5.5 будет проведено в предположении, что все параметры $\xi_{2}, \xi_{3}, \eta_{1}, \eta_{3}, \zeta_{1}, \zeta_{2}$, определяющие размеры $\Pi$, равны единице. В общем случае рассуждения будут теми же.

\section{2. Свойства построенного разбиения.}

Лемма 5.6. Пусть $X-$ приведенная матрица. Тогда

$$
\frac{q}{2 a} \leqslant b \leqslant \frac{4 q}{a}, \quad \frac{P}{24 q} \leqslant c \leqslant \frac{2 P}{q} .
$$

ДокаЗАТЕЛЬСтво. Утверждение леммы следует из неравенств $q \leqslant 2 a b$, $a b c \leqslant P \leqslant 6 a b c$ и свойства $2^{\circ}$ приведенных матриц.

Лемма 5.7. Разбиение, построенное в лемме 5.2, дополнительно обладает следующими свойствами: любое из множеств $\mathscr{M}_{\ell}(a, q, P)$ задается конечным числом неравенств вида $\pm c_{2} \leqslant f_{i}\left(a_{2}, a_{3}, b_{1}, b_{3}, c_{1}\right)\left(1 \leqslant i \leqslant i_{0}\right)$, каждое из которых действует над соответствующей областью $\Omega_{i}=\Omega_{i}\left(a_{2}, a_{3}, b_{1}, b_{3}, c_{1}\right)$. При этом $f_{i} \ll P / q u$

$$
\frac{\partial f_{i}}{\partial a_{2,3}} \ll \frac{P}{a U}, \quad \frac{\partial f_{i}}{\partial b_{1,3}} \ll \frac{P}{b U}, \quad \frac{\partial f_{i}}{\partial c_{1}} \ll \frac{P}{c U} \quad\left(a_{2}, a_{3}, b_{1}, b_{3}, c_{1}\right) \in \Omega_{i},
$$

где $U=\left|a_{1} b_{3}-a_{3} b_{1}\right|$. 
ДокАЗАтельство. Рассмотрим матрицы Минковского I типа. Очевидно, что оценка $f_{i} \ll P / q$ всегда выполняется, поскольку для приведенных матриц $c_{2} \leqslant c \asymp P / q$ (см. лемму 5.6). Рассмотрим последовательно все функции, которые могут задавать пределы изменения переменной $z_{2}$ для матриц $X$ вида (3.3), удовлетворяющих уравнению $\operatorname{det} X=P$. Это функции $f_{1,2,3,4}$, которые определяются соответственно условиями $z_{1}=z_{2}, z_{1}=z_{3}, z_{2}=z_{3}$ (возникающими при первичном разбиении множества $\mathscr{M}$ ) и $z_{3}=y_{2}$ (часть границы, возникающая из-за неравенства $b \leqslant c)$. Для первой функции $f_{1}=z_{1}$ имеем

$$
\frac{\partial f_{1}}{\partial z_{1}}=1 \ll \frac{P}{c q}, \quad \frac{\partial f_{1}}{\partial x_{2,3}}=0, \quad \frac{\partial f_{1}}{\partial y_{1,3}}=0 .
$$

Остальные функции находятся из равенства $\operatorname{det} X=P$ :

$$
\begin{gathered}
f_{2}=\frac{P-z_{1}\left(q+\left|\begin{array}{cc}
x_{2} & -x_{3} \\
y_{2} & y_{3}
\end{array}\right|\right)}{\left|\begin{array}{ll}
x_{1} & x_{3} \\
y_{1} & y_{3}
\end{array}\right|}, \quad f_{3}=\frac{P-z_{1}\left|\begin{array}{cc}
x_{2} & -x_{3} \\
y_{2} & y_{3}
\end{array}\right|}{q+\left|\begin{array}{ll}
x_{1} & x_{3} \\
y_{1} & y_{3}
\end{array}\right|}, \\
f_{4}=\frac{P-y_{2} q-z_{1}\left|\begin{array}{cc}
x_{2} & -x_{3} \\
y_{2} & y_{3}
\end{array}\right|}{\left|\begin{array}{ll}
x_{1} & x_{3} \\
y_{1} & y_{3}
\end{array}\right|} .
\end{gathered}
$$

(Если функция $f_{i}$, где $i=1, \ldots, 4$, задает границу области изменения $c_{2}$, то, как отмечалось выше, $f_{i} \ll P / q$, поэтому знаменатель $U=\left|a_{1} b_{3}-a_{3} b_{1}\right|$ в таких случаях отличен от нуля.) Если $f_{i}=F_{i} / G_{i} \ll P / q$, то

$$
f_{i}^{\prime}=\frac{F_{i}^{\prime}}{G_{i}}-\frac{F_{i} G_{i}^{\prime}}{G_{i}^{2}} \ll\left|\frac{F_{i}^{\prime}}{G_{i}}\right|+\frac{P}{q}\left|\frac{G_{i}^{\prime}}{G_{i}}\right| .
$$

Для всех рассматриваемых функций

$$
\begin{array}{rlrl}
\frac{\partial F_{i}}{\partial x_{j}} \ll \frac{P}{a}, & \frac{\partial F_{i}}{\partial y_{j}} \ll \frac{P}{b}, & & \frac{\partial F_{i}}{\partial z_{1}} \ll \frac{P}{c}, \\
\frac{\partial G_{i}}{\partial x_{j}} \ll \frac{q}{a}, & \frac{\partial G_{i}}{\partial y_{j}} \ll \frac{q}{b}, & \frac{\partial G_{i}}{\partial z_{1}}=0 .
\end{array}
$$

Значит, для проверки утверждения леммы достаточно убедиться в том, что $\left|G_{i}\right| \gg U$. Для функций $f_{2}$ и $f_{4}$ это очевидно, а для функции $f_{3}$ следует из неравенств на элементы матрицы Минковского:

$$
q+\left|\begin{array}{ll}
x_{1} & x_{3} \\
y_{1} & y_{3}
\end{array}\right|=x_{1} y_{2}+x_{2} y_{1}+x_{1} y_{3}-x_{3} y_{1} \geqslant x_{1} y_{2} \geqslant \frac{q}{2} \gg U
$$

(при $x_{2} \geqslant x_{3}$ это следует из неравенства $x_{2} y_{1}-x_{3} y_{1} \geqslant 0$, при $y_{3} \geqslant y_{1}-$ из неравенства $x_{1} y_{3}-x_{3} y_{1} \geqslant 0$, а при $z_{1} \geqslant z_{2}$ функция $f_{2}$ не может задавать границу области изменения $c_{2}$, поскольку тогда внутри этой области выполнялось бы неравенство $z_{1}>z_{2}$, противоречащее условию $z_{1}=z_{3}$, которое определяет $f_{2}$ ).

Для матриц II типа единственное отличие в доказательстве оценок (5.6) будет заключаться в необходимости рассматривать функцию $f_{5}$, которая задается условием $z_{1}+z_{2}=z_{3}$. Для нее проверка условий (5.6) проводится так же, как и для остальных функций. Лемма доказана. 
5.3. Схема доказательства основного результата. Для доказательства равенства (5.4) представим множество $\mathscr{M}_{\ell}(P)$ в виде

$$
\mathscr{M}_{\ell}(P)=\bigsqcup_{a, q} \mathscr{M}_{\ell}(a, q, P)
$$

где $\mathscr{M}_{\ell}(a, q, P)$ - множество матриц $X \in \mathscr{M}_{\ell}(P)$, у которых фиксированы значения углового элемента $a_{1}=a$ и углового минора $\operatorname{det} A=q$. Тогда

$$
\mathscr{N}_{\ell}(a, q, P)=\left|\mathscr{M}_{\ell}(a, q, P)\right|=\sum_{a_{2}, a_{3}, b_{1}, b_{3}, c_{1}, c_{2}}^{\#}\left[X \in \mathscr{M}_{\ell}(a, q, P)\right] .
$$

В последнем равенстве предполагается, что при суммировании по $a_{2}, a_{3}, b_{1}$, $b_{3}, c_{1}, c_{2}$ значения $b_{2}$ и $c_{3}$ находятся из равенств $\left|\begin{array}{ll}a_{1} & a_{2} \\ b_{1} & b_{2}\end{array}\right|=q$ и $\operatorname{det} X=P$ соответственно.

В процессе доказательства от суммирования по переменным $a_{2}, a_{3}, b_{1}, b_{3}$, $c_{1}, c_{2}$ будет последовательно (в разном порядке) делаться переход к интегрированию по переменным $\alpha_{i}=a_{i} a_{1}^{-1}, \beta_{i}=b_{i} b_{2}^{-1}, \gamma_{i}=c_{i} c_{3}^{-1}$. В итоге это позволит преобразовать сумму (5.7) в интеграл (5.5).

Определим параметры $\varkappa$ и $\lambda$ равенствами $a=P^{\varkappa}$ и $q=P^{\lambda}$. Дополнительно разобьем область

$$
\Omega=\left\{(a, q): a^{2} \ll q \ll a^{1 / 2} P^{1 / 2}\right\}=\left\{(a, q): 0 \leqslant \varkappa \leqslant \frac{1}{3}, 2 \varkappa \leqslant \lambda \leqslant \frac{1+\varkappa}{2}\right\},
$$

внутри которой меняются пары $(a, q)$, на три части в зависимости от значений $\varkappa$ и $\lambda$ - см. рис. 10.

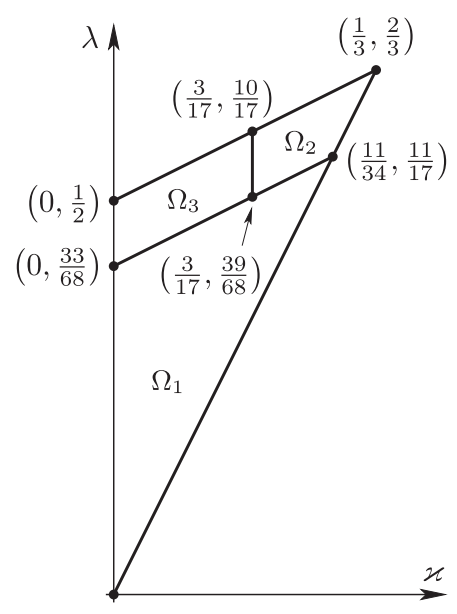

$$
\begin{aligned}
& \Omega=\Omega_{1} \sqcup \Omega_{2} \sqcup \Omega_{3} ; \\
& \Omega_{1}=\left\{(a, q) \in \Omega: \lambda \leqslant \frac{33}{68}+\frac{\varkappa}{2}\right\} ; \\
& \Omega_{2}=\left\{(a, q) \in \Omega: \varkappa \geqslant \frac{3}{17}, \lambda>\frac{33}{68}+\frac{\varkappa}{2}\right\} ; \\
& \Omega_{3}=\left\{(a, q) \in \Omega: \varkappa<\frac{3}{17}, \lambda>\frac{33}{68}+\frac{\varkappa}{2}\right\} .
\end{aligned}
$$

Рис. 10. Разбиение области изменения параметров $\varkappa$ и $\lambda$

Асимптотические формулы для $\mathscr{N}_{\ell}(a, q, P)$ будем доказывать тремя разными способами в зависимости от того, какой из областей $\Omega_{i}(i=1,2,3)$ принадлежит пара $(a, q)$. 
1. При малых значениях $a$ и $b$ (т. е. при $\left.(a, q) \in \Omega_{1}\right)$ уравнение (1.2) сначала решается как линейное относительно $c_{1}, c_{2}, c_{3}$ (см. раздел 6). Решения подсчитываются с помощью элементарных соображений. Происходит редукция к двумерной задаче, которая решается методами раздела 4.

2. Если значения $a, b, c$ соизмеримы (т. е. при $\left.(a, q) \in \Omega_{2}\right)$, то фиксируется матрица $A=\left(\begin{array}{ll}a_{1} & a_{2} \\ b_{1} & b_{2}\end{array}\right)$ и уравнение (1.2) решается относительно неизвестных $a_{3}, b_{3}, c_{1}, c_{2}, c_{3}$ (см. раздел 7 ). Решения подсчитываются с помощью нелинейной параметризации Линника-Скубенко и оценок сумм Клостермана. Снова происходит редукция к двумерной задаче, которая также решается с помощью методов раздела 4.

3. В оставшемся случае, когда $a$ мало́, а $b$ и $c$ - большие (т. е. $\left.(a, q) \in \Omega_{3}\right)$, уравнение (1.2) решается относительно неизвестных $b_{3}, c_{1}, c_{2}, c_{3}$ (см. раздел 8). Сведение к двумерной задаче осуществляется с помощью специальной версии редукции Линника-Скубенко, сохраняющей значение $a_{3}$. Возникающая двумерная задача решается элементарными методами.

Величина $\mathscr{N}_{\ell}(P)$, соответственно, представляется в виде

$$
\mathscr{N}_{\ell}(P)=\mathscr{N}_{\ell}^{(1)}(P)+\mathscr{N}_{\ell}^{(2)}(P)+\mathscr{N}_{\ell}^{(3)}(P),
$$

где

$$
\mathscr{N}_{\ell}^{(i)}(P)=\sum_{(a, q) \in \Omega_{i}} \mathscr{N}_{\ell}(a, q, P) .
$$

Для каждого случая укажем порядок переходов от суммирования к интегрированию и номера утверждений, в которых эти переходы осуществляются.

1. $\left(c_{1}, c_{2}\right)$ - леммы $6.2,6.3$ и $6.4 ; b_{3}$ - лемма $6.7 ; b_{1}$ - лемма $6.9 ;\left(a_{2}, a_{3}\right)$ лемма $9.13 ; q$ - предложение 9.14 .

2. $\left(c_{1}, c_{2}, a_{3}, b_{3}\right)$ - теорема 7.1 и следствие $7.3 ;\left(a_{2}, b_{1}\right)$ - предложение $7.7 ; q-$ предложение 9.12.

3. $\left(c_{1}, c_{2}, b_{3}\right)$ - теорема 8.1 и следствие $8.2 ; b_{1}$ - предложение $8.3 ;\left(a_{2}, a_{3}\right)$ лемма $9.13 ; q$ - предложение 9.14 .

После этого три полученных результата подставляются в равенство (5.8). В доказательстве теоремы 5.5 делается последний переход от суммирования к интегрированию по переменной $a_{1}$.

После перехода к матрицам, в которых часть коэффициентов становится действительными числами, символ \# будет означать, что вместо условий вполне примитивности (2.9)-(2.11) выполняются лишь те необходимые ограничения, которые имеют смысл (т. е. те, в которых участвуют только целые числа):

$$
\begin{array}{ll}
\left(\begin{array}{lll}
\mathbb{Z} & \mathbb{Z} & \mathbb{Z} \\
\mathbb{Z} & \mathbb{Z} & \mathbb{Z} \\
\mathbb{R} & \mathbb{R} & \mathbb{R}
\end{array}\right): & \left(\begin{array}{ll}
\mid a_{1} & a_{2} \\
b_{1} & b_{2}
\end{array}|,| \begin{array}{ll}
a_{2} & a_{3} \\
b_{2} & b_{3}
\end{array}|,| \begin{array}{ll}
a_{1} & a_{3} \\
b_{1} & b_{3}
\end{array} \mid\right)=1 ; \\
\left(\begin{array}{lll}
\mathbb{Z} & \mathbb{Z} & \mathbb{Z} \\
\mathbb{Z} & \mathbb{Z} & \mathbb{R} \\
\mathbb{R} & \mathbb{R} & \mathbb{R}
\end{array}\right): \quad & \left(a_{1}, a_{2}, a_{3}\right)=1, \quad\left(a_{1}, a_{2}, b_{1}, b_{2}\right)=1 ; \\
\left(\begin{array}{lll}
\mathbb{Z} & \mathbb{Z} & \mathbb{Z} \\
\mathbb{R} & \mathbb{R} & \mathbb{R} \\
\mathbb{R} & \mathbb{R} & \mathbb{R}
\end{array}\right): \quad & \left(a_{1}, a_{2}, a_{3}\right)=1 .
\end{array}
$$


5.4. Различные варианты сумм Клостермана. Для сумм Клостермана (1.6) известна оценка

$$
\left|K_{a}(m, n)\right| \leqslant \tau(a)(m, n, a)^{1 / 2} a^{1 / 2}
$$

$(\tau(q)$ - число делителей $q)$, которая для простых а была доказана А. Вейлем (см. [125]), а на случай произвольных а распространена Эстерманом (см. [126]).

За распределение решений уравнения (1.3) (и эквивалентного сравнения (1.4)) отвечают тригонометрические суммы (1.5), для которых удобно использовать оценку (см. [44; лемма 1])

$$
\left|K_{a}(m, n, q)\right| \leqslant \tau(a) \tau((m, n, q, a))(m n, m q, n q, a)^{1 / 2} a^{1 / 2},
$$

обобщающую неравенство (5.10).

Как отмечалось выше, при доказательстве основного результата этой статьи редукция к двумерному случаю будет проводиться тремя разными способами. В первых двух случаях возникнет необходимость в изучении решений уравнения (1.3) (сравнения (1.4)) при дополнительных условиях $(a, x, y, z)=1$ или $(a, x)=1$. Соответствующие тригонометрические суммы определяются равенствами

$$
\begin{aligned}
& K_{a}^{\times}(m, n, q)=\sum_{x, y=1}^{a} \sum_{z}[a z-x y=q,(a, x, y, z)=1] e\left(\frac{m x+n y}{a}\right) \\
& K_{a}^{*}(m, n, q)=\sum_{x=1}^{a} \sum_{y=1}^{a} \delta_{a}(x y+q) e\left(\frac{m x+n y}{a}\right) .
\end{aligned}
$$

Оценки сумм (5.12) и (5.13) сводятся к оценке (5.11), см. [24; леммы 1-3]. В обоих случаях это позволяет доказать равномерную распределенность решений сравнения $x y+q \equiv 0(\bmod a)$ с соответствующими ограничениями (см. предложения 6.8 и 7.4 ниже). Для краткости будем использовать обозначение $K_{a}^{\times}(q)=K_{a}^{\times}(0,0, q)$.

\section{6. Первый вариант оценки остатка}

В этом разделе редукция к двумерному случаю осуществляется с помощью элементарных соображений. Для двумерного случая аналогичные рассуждения были описаны в пп. 4.3, 4.4. При переходе к интегрированию во второй строке матрицы $X$ применяются стандартные “двумерные” методы, основанные на оценках сумм Клостермана (см. п. 4.5).

\section{1. Линейная параметризация решений.}

ЛЕмма 6.1. Матрица $A=\left(\begin{array}{ll}a_{1} & a_{2} \\ b_{1} & b_{2}\end{array}\right)$ с определителем $q \neq 0$ может быть дополнена до матрищъ

$$
\bar{A}=\left(\begin{array}{lll}
a_{1} & a_{2} & a_{3} \\
b_{1} & b_{2} & b_{3}
\end{array}\right),
$$


удовлетворяющей условию

$$
\left(q,\left|\begin{array}{ll}
a_{2} & a_{3} \\
b_{2} & b_{3}
\end{array}\right|,\left|\begin{array}{ll}
a_{1} & a_{3} \\
b_{1} & b_{3}
\end{array}\right|\right)=1
$$

тогда и только тогда, когда $\left(a_{1}, a_{2}, b_{1}, b_{2}\right)=1$.

Для произвольной матрицы $\bar{A}$, удовлетворяющей условию (6.2), можно найти такие челые числа $c_{1}, c_{2}, c_{3}$, что будет выполняться равенство

$$
\left|\begin{array}{lll}
a_{1} & a_{2} & a_{3} \\
b_{1} & b_{2} & b_{3} \\
c_{1} & c_{2} & c_{3}
\end{array}\right|=1
$$

Доказательство см. в [23].

Лемма 6.2. Если матрица (6.1) удовлетворяет условию (6.2) и ( $\left.\widetilde{c}_{1}, \widetilde{c}_{2}, \widetilde{c}_{3}\right)$ частное решение уравнения (6.3), то справедливы следующие утверждения.

(i) Все решения уравнения

$$
\left|\begin{array}{lll}
a_{1} & a_{2} & a_{3} \\
b_{1} & b_{2} & b_{3} \\
c_{1} & c_{2} & c_{3}
\end{array}\right|=P
$$

относительно неизвестных $c_{1}, c_{2}, c_{3}$ имеют вид

$$
\left(\begin{array}{l}
c_{1} \\
c_{2} \\
c_{3}
\end{array}\right)=P\left(\begin{array}{l}
\widetilde{c}_{1} \\
\widetilde{c}_{2} \\
\widetilde{c}_{3}
\end{array}\right)+u\left(\begin{array}{l}
a_{1} \\
a_{2} \\
a_{3}
\end{array}\right)+v\left(\begin{array}{l}
b_{1} \\
b_{2} \\
b_{3}
\end{array}\right) \quad(u, v \in \mathbb{Z}) .
$$

(ii) Для разных пар $(u, v)$ формула (6.5) дает разные решения $\left(c_{1}, c_{2}, c_{3}\right)$.

(iii) Решение $\left(c_{1}, c_{2}, c_{3}\right)$, полученное по формуле (6.5), задает вполне примитивную матрицу (1.8) тогда и только тогда, когда $(u, P)=(v, P)=1$.

ДокАЗАтЕльство. (i) Произвольный целочисленный вектор $c=\left(c_{1}, c_{2}, c_{3}\right)$ может быть представлен в виде линейной комбинации векторов $a=\left(a_{1}, a_{2}, a_{3}\right)$, $b=\left(b_{1}, b_{2}, b_{3}\right)$ и $\widetilde{c}=\left(\widetilde{c}_{1}, \widetilde{c}_{2}, \widetilde{c}_{3}\right)$ с целыми коэффициентами. Из равенства $(6.4)$ следует, что коэффициент при $\widetilde{c}$ должен быть равен $P$. Это доказывает возможность представления в виде (6.5).

(ii) Из условия (6.2) следует линейная независимость векторов $a$ и $b$. Значит, разным парам $(u, v)$ соответствуют разные решения $\left(c_{1}, c_{2}, c_{3}\right)$.

(iii) Последнее утверждение леммы проверяется с помощью формул (6.5) и $(6.2)$ :

$$
\begin{aligned}
& \left(\left|\begin{array}{ll}
a_{1} & a_{2} \\
c_{1} & c_{2}
\end{array}\right|,\left|\begin{array}{ll}
a_{1} & a_{3} \\
c_{1} & c_{3}
\end{array}\right|,\left|\begin{array}{cc}
a_{2} & a_{3} \\
c_{2} & c_{3}
\end{array}\right|\right)=\left(\left|\begin{array}{cc}
a_{1} & a_{2} \\
c_{1} & c_{2}
\end{array}\right|,\left|\begin{array}{ll}
a_{1} & a_{3} \\
c_{1} & c_{3}
\end{array}\right|,\left|\begin{array}{ll}
a_{2} & a_{3} \\
c_{2} & c_{3}
\end{array}\right|, P\right) \\
& =\left(v\left|\begin{array}{ll}
a_{1} & a_{2} \\
b_{1} & b_{2}
\end{array}\right|, v\left|\begin{array}{ll}
a_{1} & a_{3} \\
b_{1} & b_{3}
\end{array}\right|, v\left|\begin{array}{ll}
a_{2} & a_{3} \\
b_{2} & b_{3}
\end{array}\right|, P\right)=(v, P), \\
& \left(\left|\begin{array}{ll}
b_{1} & b_{2} \\
c_{1} & c_{2}
\end{array}\right|,\left|\begin{array}{ll}
b_{1} & b_{3} \\
c_{1} & c_{3}
\end{array}\right|,\left|\begin{array}{ll}
b_{2} & b_{3} \\
c_{2} & c_{3}
\end{array}\right|\right)=\left(\left|\begin{array}{ll}
b_{1} & b_{2} \\
c_{1} & c_{2}
\end{array}\right|,\left|\begin{array}{ll}
b_{1} & b_{3} \\
c_{1} & c_{3}
\end{array}\right|,\left|\begin{array}{ll}
b_{2} & b_{3} \\
c_{2} & c_{3}
\end{array}\right|, P\right) \\
& =\left(u\left|\begin{array}{ll}
a_{1} & a_{2} \\
b_{1} & b_{2}
\end{array}\right|, u\left|\begin{array}{ll}
a_{1} & a_{3} \\
b_{1} & b_{3}
\end{array}\right|, u\left|\begin{array}{ll}
a_{2} & a_{3} \\
b_{2} & b_{3}
\end{array}\right|, P\right)=(u, P) \text {. }
\end{aligned}
$$


Поэтому матрица $X$ будет вполне примитивной тогда и только тогда, когда $(u, P)=(v, P)=1$. Лемма доказана.

Предположим, что $\Omega$ - плоская область со спрямляемой границей. Пусть $\operatorname{Area}(\Omega)$ обозначает площадь этой области, $\mathscr{P}(\Omega)$ - ее периметр и $N(\Omega)$ - число точек решетки $\mathbb{Z}^{2}$, лежащих внутри $\Omega$. Для выпуклой области известно неравенство Ярника

$$
|\operatorname{Area}(\Omega)-N(\Omega)|<\mathscr{P}(\Omega)+1
$$

Для произвольной плоской односвязной области $\Omega$ со спрямляемой границей (см., например, [67; лемма 1])

$$
|\operatorname{Area}(\Omega)-N(\Omega)|<4(\mathscr{P}(\Omega)+1) \text {. }
$$

Лемма 6.3. Пусть $P$ - натуральное число, $\Omega$ - плоская односвязная область со спрямляемой граничей, $\mathscr{P}(\Omega) \gg 1, u$

$$
N^{*}(P ; \Omega)=\sum_{(u, v) \in \Omega}[(u, P)=(v, P)=1]
$$

Тогда

$$
N^{*}(P ; \Omega)=\frac{\varphi^{2}(P)}{P^{2}} \operatorname{Area}(\Omega)+O\left(\mathscr{P}(\Omega) P^{\varepsilon}\right) .
$$

ДокАзАтельство. По формуле обращения Мёбиуса

$$
N^{*}(P ; \Omega)=\sum_{d_{1}, d_{2} \mid P} \mu\left(d_{1}\right) \mu\left(d_{2}\right) \sum_{(u, v) \in \Omega}\left[d_{1}\left|u, d_{2}\right| v\right] .
$$

Нужная асимптотическая формула получается, если во внутренней сумме сделать замену переменных $u=d_{1} u^{\prime}, v=d_{2} v^{\prime}$, в новых переменных $u^{\prime}, v^{\prime}$ воспользоваться неравенством (6.8) и оценить периметр уменьшенной копии области $\Omega$ как $O(\mathscr{P}(\Omega))$ :

$$
\begin{aligned}
N^{*}(P ; \Omega) & =\sum_{d_{1}, d_{2} \mid P} \mu\left(d_{1}\right) \mu\left(d_{2}\right)\left(\frac{\operatorname{Area}(\Omega)}{d_{1} d_{2}}+O(\mathscr{P}(\Omega))\right) \\
& =\frac{\varphi^{2}(P)}{P^{2}} \operatorname{Area}(\Omega)+O\left(\mathscr{P}(\Omega) P^{\varepsilon}\right) .
\end{aligned}
$$

6.2. Переход к интегрированию в третьей строке. Рассмотрим множество матриц $X \in \mathscr{M}_{\ell}(a, q, P)$, для которых матрица $\bar{A}=\left(\begin{array}{lll}a_{1} & a_{2} & a_{3} \\ b_{1} & b_{2} & b_{3}\end{array}\right)$ фиксирована. Так как $q=\left|\begin{array}{ll}a_{1} & a_{2} \\ b_{1} & b_{2}\end{array}\right| \neq 0$, то значение $c_{3}$ однозначно выражается через $c_{1}$ и $c_{2}$. Поэтому любые условия, налагаемые на переменные $c_{1}, c_{2}, c_{3}$, можно записать в виде $\left(c_{1}, c_{2}\right) \in \Omega(\bar{A})$.

Лемма 6.4. Пусть $\bar{A}$ удовлетворяет условию (6.2), $\Omega$ - выпуклая область и

$$
S(\bar{A}, \Omega)=\sum_{\left(c_{1}, c_{2}\right) \in \Omega}^{\#}\left[X \in \mathscr{M}_{\ell}(a, q, P)\right]
$$


Тогда

$$
S(\bar{A}, \Omega)=\frac{\varphi^{2}(P)}{P^{2} q} \int_{\Omega}\left[X \in \mathscr{M}_{\ell}(a, q, P)\right] d c_{1} d c_{2}+O\left(\frac{P^{1+\varepsilon}}{a q}\right) .
$$

ДокАЗАТЕЛЬСтво. По построению множество $\mathscr{M}_{\ell}(a, q, P)$ состоит из матриц вида (3.3), (3.4), (5.1) или (5.2). Поэтому переменные $c_{1}, c_{2}, c_{3}$ должны удовлетворять некоторым условиям из следующего списка:

$$
0 \leqslant c_{1} \leqslant\left|c_{2}\right| \leqslant c_{3}, \quad 0 \leqslant\left|c_{2}\right| \leqslant c_{1} \leqslant c_{3}, \quad c_{1}+\left|c_{2}\right| \geqslant c_{3} .
$$

Значит, область изменения переменных $c_{1}, c_{2}$ содержится в выпуклом многоугольнике, линейные размеры которого по лемме 5.6 оцениваются как $O(c)=$ $O(P / q)$. Из равенства (6.5) следует, что линейные размеры соответствующего многоугольника на плоскости Ouv суть $O\left(b P / q^{2}\right)$. По лемме 6.3,

$$
\begin{aligned}
S(\bar{A}, \Omega) & =\sum_{\substack{u, v \\
(u, P)=(v, P)=1}}^{\#}\left[X \in \mathscr{M}_{\ell}(a, q, P),\left(c_{1}, c_{2}\right) \in \Omega\right] \\
& =\frac{\varphi^{2}(P)}{P^{2}} \int_{\mathbb{R}^{2}}\left[X \in \mathscr{M}_{\ell}(a, q, P),\left(c_{1}, c_{2}\right) \in \Omega\right] d u d v+O\left(\frac{P^{1+\varepsilon}}{a q}\right) \\
& =\frac{\varphi^{2}(P)}{P^{2} q} \int_{\Omega}\left[X \in \mathscr{M}_{\ell}(a, q, P)\right] d c_{1} d c_{2}+O\left(\frac{P^{1+\varepsilon}}{a q}\right) .
\end{aligned}
$$

СлеДСТвиЕ 6.5. Пусть выполняются условия леммы 6.4. Тогда

$$
S(\bar{A}, \Omega) \ll \frac{P^{2+\varepsilon}}{q^{3}} .
$$

Кроме того, при фиксированнъх $\widetilde{a}_{3}$ и $\widetilde{b}_{3}$

$$
\sum_{\left(c_{1}, c_{2}\right) \in\left(\tilde{u}_{3}, b_{3}\right)}^{\#}\left[X \in \mathscr{M}_{\ell}(a, q, P)\right]
$$

$\left(c_{1}, c_{2}\right) \in \Omega\left(\widetilde{a}_{3}, \widetilde{b}_{3}\right)$

$$
=\frac{\varphi^{2}(P)}{P^{2} q} \int_{\widetilde{a}_{3}}^{\widetilde{a}_{3}+1} d a_{3} \int_{\widetilde{b}_{3}}^{\widetilde{b}_{3}+1} d b_{3} \sum_{\left(c_{1}, c_{2}\right) \in \Omega\left(a_{3}, b_{3}\right)}\left[X \in \mathscr{M}_{\ell}(a, q, P)\right]+O\left(\frac{P^{2+\varepsilon}}{q^{3}}\right) .
$$

ДокАЗАтЕльство. Для проверки оценки (6.9) достаточно тривиальным образом оценить интеграл из леммы 6.4. Равенство (6.10) следует из того, что каждое слагаемое в нем есть $O\left(P^{2+\varepsilon} q^{-3}\right)$.

6.3. Переход к интегрированию во второй строке. Под нормой функции всегда будем понимать $L^{\infty}$-норму.

Лемма 6.6. Пусть $а, D$ - натуралъные числа и $D \mid$ a. Предположим, что функиия $f$ на интервале I имеет конечное число участков монотонности. Тогда справедливы следующие асимптотические формуль:

$$
\sum_{\substack{x \in I \\ x \in x_{0}(\bmod D)}} f(x)=\frac{1}{D} \int_{I} f(x) d x+O(\|f\|),
$$




$$
\begin{aligned}
& \sum_{\substack{x \in I \\
(x, a)=D}} f(x)=\frac{\varphi(a / D)}{a} \int_{I} f(x) d x+O\left(\|f\| a^{\varepsilon}\right), \\
& \sum_{\substack{x \in I \\
(x, a)=1}} f(x)=\frac{\varphi(a)}{a} \int_{I} f(x) d x+O\left(\|f\| a^{\varepsilon}\right) .
\end{aligned}
$$

Доказательство см. в [24; лемма 4].

Лемма 6.7. Пусть $A=\left(\begin{array}{ll}a_{1} & a_{2} \\ b_{1} & b_{2}\end{array}\right), q=\operatorname{det} A>0,\left(a_{1}, a_{2}, b_{1}, b_{2}\right)=1, D=$ $\left(a_{1}, a_{2}\right),\left(D, a_{3}\right)=1$ и для всех $n$ из интервала $I$

$$
f(n)=\left|\begin{array}{ccc}
a_{1} & a_{2} & a_{3} \\
b_{1} & b_{2} & n \\
z_{1} & z_{2} & 1
\end{array}\right|^{-3} \ll \frac{1}{q^{3}}
$$

Тогда для суммы

$$
S^{\#}(I)=\sum_{n \in I}^{\#} f(n)
$$

справедлива асимптотическая формула

$$
S^{\#}(I)=\frac{\varphi(q)}{q} \frac{D}{\varphi(D)} \int_{I} f(t) d t+O\left(q^{-3+\varepsilon}\right) .
$$

ДокАЗАТЕЛьСтво. Пусть матрица $A$ элементарными преобразованиями столбцов приводится к виду $\left(\begin{array}{cc}D & 0 \\ \alpha & q D^{-1}\end{array}\right)$. Тогда условие (6.2) равносильно равенству

$$
\left(q, \frac{q}{D} a_{3},\left|\begin{array}{cc}
D & a_{3} \\
\alpha & n
\end{array}\right|\right)=1 \text {. }
$$

Так как $\left(D, a_{3}\right)=1$, то $\left(q,(q / D) a_{3}\right)=(q / D)\left(D, a_{3}\right)=q / D$ и равенство $(6.14)$ означает, что $\left(\frac{q}{D},\left|\begin{array}{cc}D & a_{3} \\ \alpha & n\end{array}\right|\right)=1$. Значит,

$$
S^{\#}(I)=\sum_{n \in I}\left[\left(\frac{q}{D},\left|\begin{array}{cc}
D & a_{3} \\
\alpha & n
\end{array}\right|\right)=1\right] f(n)=\sum_{\delta \mid q / D} \mu(\delta) \sum_{n \in I}\left[\delta \mid\left(D n-\alpha a_{3}\right)\right] f(n) .
$$

Из условия $\delta \mid\left(D n-\alpha a_{3}\right)$ следует, что $(\delta, D) \mid \alpha a_{3}$. По условию леммы $\left(D, a_{3}\right)=1$, значит, $(\delta, D) \mid \alpha$. Kроме того, $(D, q / D, \alpha)=\left(a_{1}, a_{2}, b_{1}, b_{2}\right)=1$. Поэтому из соотношений $(\delta, D) \mid \alpha$ и $\delta \mid q / D$ следует, что $(\delta, D)=1$. При $(\delta, D)=1$ сравнение $D n-\alpha a_{3} \equiv 0(\bmod \delta)$ равносильно условию $n \equiv n_{0}$ $(\bmod \delta)$, где $n_{0} \equiv \alpha a_{3} D^{-1}(\bmod \delta)$. Для завершения доказательства леммы остается воспользоваться равенством (6.11):

$$
\begin{aligned}
S^{\#}(I) & =\sum_{\substack{\delta \mid q / D \\
(\delta, D)=1}} \mu(\delta) \sum_{\substack{n \in I \\
n \equiv n_{0}(\bmod \delta)}} f(n)=\sum_{\substack{\delta \mid q / D \\
(\delta, D)=1}} \mu(\delta)\left(\frac{1}{\delta} \int_{I} f(t) d t+O\left(q^{-3}\right)\right) \\
& =\sum_{\substack{\delta \mid q / D \\
(\delta, D)=1}} \frac{\mu(\delta)}{\delta} \int_{I} f(t) d t+O\left(q^{-3+\varepsilon}\right)=\frac{\varphi(q)}{q} \frac{D}{\varphi(D)} \int_{I} f(t) d t+O\left(q^{-3+\varepsilon}\right) .
\end{aligned}
$$


Если $(x, a)=1$, то каждый интервал $[Y, Y+a)$ содержит ровно одно решение сравнения $x y+q \equiv 0(\bmod a)$ относительно неизвестной $y$. Поэтому для произвольной функции $G$, определенной на прямоугольнике $\left[Y_{1}, Y_{1}+Z_{1}\right) \times\left[Y_{2}, Y_{2}+Z_{2}\right)$, естественной аппроксимацией для суммы

$$
\Phi_{a, q}^{*}[G]=\sum_{Y_{1} \leqslant x<Y_{1}+Z_{1}}^{*} \sum_{Y_{2} \leqslant y<Y_{2}+Z_{2}} \delta_{a}(x y+q) G(x, y)
$$

является сумма

$$
S_{a}^{*}[G]=\frac{1}{a} \sum_{Y_{1} \leqslant x<Y_{1}+Z_{1}}^{*} \int_{Y_{2}}^{Y_{2}+Z_{2}} G(x, y) d y .
$$

ПредложЕНИЕ 6.8. Пусть $G$ - неотрищательная функиия и для любого z в пределах $0 \leqslant z \leqslant\|G\|$ неравенство $G(x, y) \leqslant z$ задает в прямоугольнике $I=[0, a] \times\left[0, Z_{2}\right]\left(Z_{2} \ll q / a\right)$ область $\Omega_{z}=\left\{(x, y) \in I: y \leqslant f_{z}(x)\right\}$, причем число участков монотонности для всех функиий $f_{z}$ ограничено абсолютной константой. Тогда

$$
\Phi_{a, q}^{*}[G]-S_{a}^{*}[G] \ll\|G\|(a, q) a^{-1 / 4} q^{1 / 2+\varepsilon} .
$$

Доказательство см. в [24; теорема 4].

Лемма 6.9. Пусть $a_{1}=a$ и $D \mid(a, q)$. Тогда для суммы

$$
S(a, q, D)=\sum_{\substack{a_{2}, a_{3}, b_{1} \\\left(a_{1}, a_{2}\right)=D}}^{\#} \int_{\mathbb{R}}\left[X^{\prime} \in \mathscr{M}_{\ell}^{\prime}(a, q, P)\right]\left(\operatorname{det} X^{\prime}\right)^{-3} d b_{3}
$$

справедлива асимптотическая формула

$$
\begin{gathered}
S(a, q, D)=\frac{D \varphi((D, q / D))}{q(D, q / D)} \sum_{\substack{a_{2}, a_{3} \\
\left(a, a_{2}\right)=D}}^{\#} \int_{\mathbb{R}^{2}}\left[X^{\prime \prime} \in \mathscr{M}_{\ell}^{\prime \prime}(a, q, P)\right]\left(\operatorname{det} X^{\prime \prime}\right)^{-3} d \beta_{1} d \beta_{3} \\
+O\left((a, q) a^{-1 / 4} q^{-3 / 2+\varepsilon}\right) .
\end{gathered}
$$

ДокАЗАТЕЛЬСтво. Преобразуем указанную сумму, вводя переменные $a_{1}^{\prime}=$ $a_{1} D^{-1}, a_{2}^{\prime}=a_{2} D^{-1}, a_{3}^{\prime}=a_{3} D^{-1}\left(a_{3}^{\prime}-\right.$ не обязательно целое):

$$
S(a, q, D)=\frac{1}{D^{3}} \int_{\mathbb{R}} d b_{3} \sum_{\substack{a_{3} \\
\left(a_{3}, D\right)=1}} \sum_{a_{2}^{\prime}}^{*} \sum_{\substack{b_{1} \\
\left(D, b_{1}, b_{2}\right)=1}}\left|\begin{array}{ccc}
a_{1}^{\prime} & a_{2}^{\prime} & a_{3}^{\prime} \\
b_{1} & b_{2} & b_{3} \\
\gamma_{1} & \gamma_{2} & 1
\end{array}\right|^{-3} .
$$

Избавимся от условия $\left(D, b_{1}, b_{2}\right)=1$ с помощью функции Мёбиуса:

$$
\begin{aligned}
S\left(a_{1}, q, D\right) & =\frac{1}{D^{3}} \sum_{\delta \mid(D, q / D)} \mu(\delta) \int_{\mathbb{R}} d b_{3} \sum_{\substack{a_{3} \\
\left(a_{3}, D\right)=1}} \sum_{a_{2}^{\prime}}^{*} \sum_{b_{1}: \delta \mid\left(b_{1}, b_{2}\right)}\left|\begin{array}{ccc}
a_{1}^{\prime} & a_{2}^{\prime} & a_{3}^{\prime} \\
b_{1} & b_{2} & b_{3} \\
\gamma_{1} & \gamma_{2} & 1
\end{array}\right|^{-3} \\
& =\frac{1}{D^{3}} \sum_{\delta \mid(D, q / D)} \frac{\mu(\delta)}{\delta^{2}} \int_{\mathbb{R}} d b_{3}^{\prime} \sum_{\substack{a_{3} \\
\left(a_{3}, D\right)=1}} \sum_{a_{2}^{\prime}}^{*} \sum_{b_{1}^{\prime}}\left|\begin{array}{ccc}
a_{1}^{\prime} & a_{2}^{\prime} & a_{3}^{\prime} \\
b_{1}^{\prime} & b_{2}^{\prime} & b_{3}^{\prime} \\
\gamma_{1} & \gamma_{2} & 1
\end{array}\right|^{-3},
\end{aligned}
$$


где $b_{1}^{\prime}=b_{1} \delta^{-1}, b_{2}^{\prime}=b_{2} \delta^{-1}, b_{3}^{\prime}=b_{3} \delta^{-1}$. K внутренней двойной сумме применим предложение 6.8. По предположению леммы

$$
\left|\begin{array}{ccc}
a_{1}^{\prime} & a_{2}^{\prime} & a_{3}^{\prime} \\
b_{1}^{\prime} & b_{2}^{\prime} & b_{3}^{\prime} \\
\gamma_{1} & \gamma_{2} & 1
\end{array}\right|^{-3} \ll\left(\frac{D \delta}{q}\right)^{3}
$$

Кроме того, функция $b_{1}=f\left(a_{2}\right)$, неявно заданная равенствами

$$
\left|\begin{array}{ccc}
a_{1}^{\prime} & a_{2}^{\prime} & a_{3}^{\prime} \\
b_{1}^{\prime} & b_{2}^{\prime} & b_{3}^{\prime} \\
\gamma_{1} & \gamma_{2} & 1
\end{array}\right|=Q, \quad\left|\begin{array}{ll}
a_{1}^{\prime} & a_{2}^{\prime} \\
b_{1}^{\prime} & b_{2}^{\prime}
\end{array}\right|=q^{\prime}=\frac{q}{\Delta \delta}
$$

приводится к виду

$$
\left(a_{3}^{\prime} b_{1}^{\prime}-b_{3}^{\prime} a_{1}^{\prime}\right)\left(a_{1}^{\prime} z_{2}-a_{2}^{\prime} z_{1}\right)=a_{1}^{\prime} Q-q^{\prime}\left|\begin{array}{cc}
a_{1}^{\prime} & a_{3}^{\prime} \\
z_{1} & 1
\end{array}\right| .
$$

Поэтому график $f$ состоит из конечного числа участков монотонности и предложение 6.8 действительно можно применять. Значит,

$$
\begin{aligned}
S\left(a_{1}, q, D\right)=\frac{1}{D^{3}} & \sum_{\delta \mid(D, q / D)} \frac{\mu(\delta)}{\delta^{2}} \int_{\mathbb{R}} d b_{3}^{\prime} \sum_{\substack{a_{3} \\
\left(a_{3}, D\right)=1}}\left(\frac{1}{a_{1}^{\prime}} \sum_{a_{2}^{\prime}}^{*} \int_{\mathbb{R}}\left|\begin{array}{ccc}
a_{1}^{\prime} & a_{2}^{\prime} & a_{3}^{\prime} \\
b_{1}^{\prime} & b_{2}^{\prime} & b_{3}^{\prime} \\
\gamma_{1} & \gamma_{2} & 1
\end{array}\right|^{-3} d b_{1}^{\prime}\right. \\
& \left.+O\left(\left(\frac{D \delta}{q}\right)^{3}\left(\frac{a}{D}, \frac{q}{D \delta}\right)\left(\frac{a}{D}\right)^{-1 / 4}\left(\frac{q}{D \delta}\right)^{1 / 2+\varepsilon}\right)\right)
\end{aligned}
$$

Просуммируем возникшие остатки. Переменная $b_{3}^{\prime}$ меняется в интервале длины $O(q /(a \delta))$, переменная $a_{3}-$ в интервале длины $O(a)$. Поэтому сумма остатков оценивается суммой

$$
\sum_{\delta \mid(D, q / D)}(a, q) a^{-1 / 4} D^{-1 / 4} \delta^{-1 / 2} q^{-3 / 2+\varepsilon} \ll(a, q) a^{-1 / 4} q^{-3 / 2+\varepsilon} .
$$

Преобразуем сумму главных членов, переходя к переменным $a_{2}=D a_{2}^{\prime}$ и $a_{3}=D a_{3}^{\prime}$ :

$$
\begin{gathered}
\sum_{\delta \mid(D, q / D)} \frac{\mu(\delta)}{\delta^{2}} \int_{\mathbb{R}} \frac{d b_{3}}{\delta} \sum_{\substack{a_{3} \\
\left(a_{3}, D\right)=1}} \frac{D}{a_{1}} \sum_{\substack{a_{2} \\
\left(a, a_{2}\right)=D}} \int_{\mathbb{R}} \delta^{2}\left|\begin{array}{ccc}
a_{1} & a_{2} & a_{3} \\
b_{1} & b_{2} & b_{3} \\
\gamma_{1} & \gamma_{2} & 1
\end{array}\right|^{-3} d b_{1} \\
=\frac{D}{a} \sum_{\delta \mid(D, q / D)} \frac{\mu(\delta)}{\delta} \sum_{\substack{a_{2}, a_{3} \\
\left(a, a_{2}\right)=D}}^{\#} \int_{\mathbb{R}^{2}}\left|\begin{array}{ccc}
a_{1} & a_{2} & a_{3} \\
b_{1} & b_{2} & b_{3} \\
\gamma_{1} & \gamma_{2} & 1
\end{array}\right|^{-3} d b_{1} d b_{3} .
\end{gathered}
$$


После этого остается сделать переход к переменным $\beta_{1}=b_{1} / b_{2}, \beta_{3}=b_{3} / b_{2}$, где $b_{2}=\left(q+a_{2} b_{1}\right) a_{1}^{-1}$. Так как

$$
\frac{\partial\left(b_{1}, b_{3}\right)}{\partial\left(\beta_{1}, \beta_{3}\right)}=\frac{a b^{3}}{q}, \quad\left|\begin{array}{ccc}
a_{1} & a_{2} & a_{3} \\
b_{1} & b_{2} & b_{3} \\
\gamma_{1} & \gamma_{2} & 1
\end{array}\right|^{-3} \quad d b_{1} d b_{3}=\frac{a}{q}\left|\begin{array}{ccc}
a_{1} & a_{2} & a_{3} \\
\beta_{1} & 1 & \beta_{3} \\
\gamma_{1} & \gamma_{2} & 1
\end{array}\right|^{-3} d \beta_{1} d \beta_{3},
$$

то получаем нужный главный член

$$
\frac{D \varphi((D, q / D))}{q(D, q / D)} \sum_{\substack{a_{2}, a_{3} \\
\left(a, a_{2}\right)=D}}^{\#} \int_{\mathbb{R}^{2}}\left|\begin{array}{ccc}
a_{1} & a_{2} & a_{3} \\
\beta_{1} & 1 & \beta_{3} \\
\gamma_{1} & \gamma_{2} & 1
\end{array}\right|^{-3} d \beta_{1} d \beta_{3} .
$$

ПредЛОЖЕНИЕ 6.10. Пусть $q \gg a^{2}$. Тогда для величины $\mathscr{N}_{\ell}(a, q, P)$, задаваемой равенством (5.7), справедлива асимптотическая формула

$$
\begin{aligned}
\mathscr{N}_{\ell}(a, q, P)= & \varphi^{2}(P)\left(\frac{\varphi(q)}{q^{2}} \int_{\mathbb{R}^{4}} d \boldsymbol{\beta} d \boldsymbol{\gamma} \sum_{a_{2}, a_{3}}^{\#} c(q, D) \frac{\left[X^{\prime \prime} \in \mathscr{M}_{\ell}^{\prime \prime}(a, q, P)\right]}{\left(\operatorname{det} X^{\prime \prime}\right)^{3}}+\rho_{1}(a, q)\right) \\
& +O\left(R_{1}(a, q, P)\right),
\end{aligned}
$$

где $D=\left(a, a_{2}\right), R_{1}(a, q, P)=a^{-2} q P^{1+\varepsilon}$,

$$
\begin{gathered}
c(q, D)=\frac{D^{2} \varphi((D, q / D))}{\varphi(D)(D, q / D)}, \\
\rho_{1}(a, q) \ll(a, q) a^{-1 / 4} q^{-3 / 2+\varepsilon} .
\end{gathered}
$$

ДокАзАтЕЛьство. Из неравенства (5.11) следует, что

$$
K_{a}^{\times}(q) \ll a^{1+\varepsilon} .
$$

Преобразуем сумму $\mathscr{N}_{\ell}(a, q, P)$, пользуясь леммой 6.4 и оценкой $(6.20)$ :

$$
\begin{aligned}
\mathscr{N}_{\ell}(a, q, P) & =\sum_{\substack{a_{2}, a_{3}, b_{1}, b_{3} \\
b_{1} a_{2}+q \equiv 0}}^{\#}\left(\frac{\varphi^{2}(P)}{P^{2} q} \int_{\mathbb{R}^{2}}\left[X \in \mathscr{M}_{\ell}(a, q, P)\right] d c_{1} d c_{2}+O\left(\frac{P^{1+\varepsilon}}{a q}\right)\right) \\
& =\frac{\varphi^{2}(P)}{P^{2} q} \sum_{a_{2}, a_{3}, b_{1}, b_{3}}^{\#} \int_{\mathbb{R}^{2}}\left[X \in \mathscr{M}_{\ell}(a, q, P)\right] d c_{1} d c_{2}+O\left(\frac{q P^{1+\varepsilon}}{a^{2}}\right) .
\end{aligned}
$$

В двойном интеграле перейдем к переменным $\gamma_{1}=c_{1} c_{3}^{-1}, \gamma_{2}=c_{2} c_{3}^{-1}$. Так как $c_{3}=P\left(\operatorname{det} X^{\prime}\right)^{-1}$, то

$$
\begin{aligned}
\frac{\partial\left(c_{1}, c_{2}\right)}{\partial\left(\gamma_{1}, \gamma_{2}\right)} & =c_{3}\left(c_{3}+\frac{\partial c_{3}}{\partial \gamma_{1}} \gamma_{1}+\frac{\partial c_{3}}{\partial \gamma_{2}} \gamma_{2}\right)=\frac{q P^{2}}{\left(\operatorname{det} X^{\prime}\right)^{3}} \\
d c_{1} d c_{2} & =\frac{q P^{2}}{\left(\operatorname{det} X^{\prime}\right)^{3}} d \gamma_{1} d \gamma_{2} .
\end{aligned}
$$

Поэтому

$$
\frac{\mathscr{N}_{\ell}(a, q, P)}{\varphi^{2}(P)}=\int_{\mathbb{R}^{2}} d \gamma \sum_{a_{2}, a_{3}, b_{1}, b_{3}}^{\#} \frac{\left[X^{\prime} \in \mathscr{M}_{\ell}^{\prime}(a, q, P)\right]}{\left(\operatorname{det} X^{\prime}\right)^{3}}+O\left(\frac{q P^{-1+\varepsilon}}{a^{2}}\right) .
$$


C помощью леммы 6.7 заменим суммирование по переменной $b_{3}$ интегрированием:

$$
\begin{aligned}
\frac{\mathscr{N}_{\ell}(a, q, P)}{\varphi^{2}(P)}= & \int_{\mathbb{R}^{2}} d \gamma \sum_{a_{2}, a_{3}, b_{1}}^{\#}\left(\frac{\varphi(q) D}{q \varphi(D)} \int_{\mathbb{R}} \frac{\left[X^{\prime} \in \mathscr{M}_{\ell}^{\prime}(a, q, P)\right]}{\left(\operatorname{det} X^{\prime}\right)^{3}} d b_{3}+O\left(q^{-3+\varepsilon}\right)\right) \\
& +O\left(\frac{q P^{-1+\varepsilon}}{a^{2}}\right) \\
= & \int_{\mathbb{R}^{2}} d \gamma \sum_{a_{2}, a_{3}, b_{1}}^{\#} \frac{\varphi(q) D}{q \varphi(D)} \int_{\mathbb{R}} \frac{\left[X^{\prime} \in \mathscr{M}_{\ell}^{\prime}(a, q, P)\right]}{\left(\operatorname{det} X^{\prime}\right)^{3}} d b_{3} \\
& +O\left(q^{-2+\varepsilon}\right)+O\left(\frac{q P^{-1+\varepsilon}}{a^{2}}\right) .
\end{aligned}
$$

Применяя лемму 6.9, приходим к равенству, равносильному формуле (6.17):

$$
\begin{aligned}
\frac{\mathscr{N}_{\ell}(a, q, P)}{\varphi^{2}(P)}= & \int_{\mathbb{R}^{2}} d \boldsymbol{\gamma} \sum_{D \mid(a, q)} \frac{\varphi(q) D}{q \varphi(D)}\left(\frac{D \varphi((D, q / D))}{q(D, q / D)}\right. \\
& \left.\times \sum_{\substack{a_{2}, a_{3} \\
\left(a, a_{2}\right)=D}}^{\#} \int_{\mathbb{R}^{2}} \frac{\left[X^{\prime \prime} \in \mathscr{M}_{\ell}^{\prime \prime}(a, q, P)\right] d \boldsymbol{\beta}}{\left(\operatorname{det} X^{\prime \prime}\right)^{3}}+O\left((a, q) a^{-1 / 4} q^{-3 / 2+\varepsilon}\right)\right) \\
& +O\left(q^{-2+\varepsilon}\right)+O\left(\frac{q P^{-1+\varepsilon}}{a^{2}}\right) \\
= & \frac{\varphi(q)}{q^{2}} \int_{\mathbb{R}^{4}} d \boldsymbol{\beta} d \boldsymbol{\gamma} \sum_{a_{2}, a_{3}}^{\#} c(q, D) \frac{\left[X^{\prime \prime} \in \mathscr{M}_{\ell}^{\prime \prime}(a, q, P)\right]}{\left(\operatorname{det} X^{\prime \prime}\right)^{3}} \\
& +O\left((a, q) a^{-1 / 4} q^{-3 / 2+\varepsilon}\right)+O\left(\frac{q P^{-1+\varepsilon}}{a^{2}}\right) .
\end{aligned}
$$

\section{7. Второй вариант оценки остатка}

Пусть $\lambda_{1}(\Lambda)$ и $\lambda_{2}(\Lambda)$ обозначают последовательные минимумы двумерной решетки $\Lambda$, т. е. $\lambda_{1}(\Lambda)$ - длина кратчайшего ненулевого вектора $e_{1} \in \Lambda$, а $\lambda_{2}(\Lambda)-$ длина кратчайшего вектора $e_{2} \in \Lambda$, линейно независимого с $e_{1}$. Через $\Lambda(A)$ обозначим решетку, состоящую из решений системы сравнений

$$
a_{1} x+a_{2} y \equiv 0 \quad(\bmod q), \quad b_{1} x+b_{2} y \equiv 0 \quad(\bmod q),
$$

и положим

$$
\lambda_{2}(A)=\lambda_{2}(\Lambda(A)), \quad \lambda_{2}\left(A^{T}\right)=\lambda_{2}\left(\Lambda\left(A^{T}\right)\right) .
$$

Теорема 7.1. Пусть матрица $A=\left(\begin{array}{ll}a_{1} & a_{2} \\ b_{1} & b_{2}\end{array}\right)$ фиксирована, $q=\operatorname{det} A \neq 0$, $\left(a_{1}, a_{2}, b_{1}, b_{2}\right)=1$ и матрица $X$ имеет вид (1.8). Тогда для любого параллелеnuneдa

$$
I=\left[Y_{1}, Y_{1}+Z_{1}\right) \times\left[Y_{2}, Y_{2}+Z_{2}\right) \times\left[Y_{3}, Y_{3}+Z_{3}\right) \times\left[Y_{4}, Y_{4}+Z_{4}\right),
$$


размеры которого удовлетворяют условиям $1 \leqslant Z_{1}, Z_{2} \leqslant q, 1 \leqslant Z_{3}, Z_{4} \leqslant P q$, выполняется равенство

$$
\sum_{\left(a_{3}, b_{3}, c_{1}, c_{2}\right) \in I} \sum_{c_{3}}^{\#}[\operatorname{det} X=P]=\frac{\varphi^{2}(P)}{P^{2}} \frac{\varphi(q)}{q^{2}} Z_{1} Z_{2} Z_{3} Z_{4}+O\left(R_{A}\left(Z_{1}, Z_{2}, Z_{3}, Z_{4}\right)\right)
$$

zдe

$$
\begin{aligned}
R_{A}\left(Z_{1}, Z_{2}, Z_{3}, Z_{4}\right)=( & P, q)(P q)^{\varepsilon}\left(\frac{Z_{1} Z_{2} Z_{3} Z_{4}}{q}\left(\frac{1}{Z_{1}}+\frac{1}{Z_{2}}+\frac{1}{Z_{3}}+\frac{1}{Z_{4}}\right)\right. \\
& +q^{1 / 2}+\frac{\lambda_{2}\left(A^{T}\right)}{q^{1 / 2}}\left(Z_{1}+Z_{2}\right)+\frac{\lambda_{2}(A)}{q^{1 / 2}}\left(Z_{3}+Z_{4}\right) \\
& \left.+\frac{\lambda_{2}(A) \lambda_{2}^{1 / 2}\left(A^{T}\right)}{q}\left(Z_{1}+Z_{2}\right)\left(Z_{3}+Z_{4}\right)\right) .
\end{aligned}
$$

ДокАЗАТЕЛЬСТво см. в [23].

ЗАмечАниЕ 7.2. Теорема 7.1 представляет собой вариант леммы 7 из работы [8]. Однако в [8] в остаточном члене не учитывался вклад слагаемых, содержащих $\lambda_{2}(A)$ и $\lambda_{2}\left(A^{T}\right)$. Дальнейшие рассуждения показывают, что именно эти слагаемые вносят основной вклад в итоговый остаток (см. выбор параметров $r_{1}$ и $r$ в доказательстве предложения 9.8). При этом используется оценка $\lambda_{2}(A) \ll q^{1 / 2+\varepsilon}$, справедливая только в среднем по $A$.

СледСтвиЕ 7.3. Пусть выполнены условия теоремы 7.1, размеры параллелепипеда

$$
I^{\prime}=\left[Y_{1}, Y_{1}+Z_{1}\right) \times\left[Y_{2}, Y_{2}+Z_{2}\right) \times\left[Y_{3}, Y_{3}+Z_{3}\right)
$$

удовлетворяют неравенствам $1 \leqslant Z_{1}, Z_{2} \leqslant q, 1 \leqslant Z_{3} \leqslant P q$ и при $\left(a_{3}, b_{3}, c_{1}\right) \in I^{\prime}$ значения неотрицательной функиии $f\left(a_{3}, b_{3}, c_{1}\right)$ меняются внутри интервала $\left[Z_{4}, Z_{4}+V\right)$, где $1 \leqslant V \ll Z_{4} \leqslant P q$. Тогда

$$
\begin{gathered}
\sum_{\left(a_{3}, b_{3}, c_{1}\right) \in I^{\prime}} \sum_{0<c_{2} \leqslant f\left(a_{3}, b_{3}, c_{1}\right)} \sum_{c_{3}}^{\#}[\operatorname{det} X=P] \\
=\frac{\varphi^{2}(P)}{P^{2}} \frac{\varphi(q)}{q^{2}} \int_{I^{\prime}} f\left(a_{3}, b_{3}, c_{1}\right) d a_{3} d b_{3} d c_{1} \\
+O\left(R_{A}\left(Z_{1}, Z_{2}, Z_{3}, Z_{4}\right)\right)+O\left(\frac{Z_{1} Z_{2} Z_{3} V}{q}\right) .
\end{gathered}
$$

ДокАЗАТЕЛЬСтво. Заменяя во второй сумме $f\left(a_{3}, b_{3}, c_{1}\right)$ на $Z_{4}$ и $Z_{4}+V$, получаем соответственно нижнюю и верхнюю оценки вычисляемой суммы. Применяя теорему 7.1 и пользуясь оценкой

$$
\int_{I^{\prime}} d x_{1} d x_{2} d x_{3}\left(f\left(x_{1}, x_{2}, x_{3}\right)-\left(Z_{4}+\theta V\right)\right) \ll Z_{1} Z_{2} Z_{3} V \quad(\theta=0,1),
$$

приходим к утверждению следствия. 
ПРЕДЛОЖЕНИЕ 7.4. Пусть а-натуральное, $q$ - иелое, $I=\left[Y_{1}, Y_{1}+Z_{1}\right) \times$ $\left[Y_{2}, Y_{2}+Z_{2}\right)$, где $Z_{1}, Z_{2}>0, u$

$$
\Phi_{a, q}^{\times}(I)=\sum_{(x, y) \in I} \sum_{z}[a z-x y=q,(a, x, y, z)=1] .
$$

Тогда

$$
\Phi_{a, q}^{\times}(I)=\frac{K_{a}^{\times}(q)}{a^{2}} Z_{1} Z_{2}+O\left(R\left(Z_{1}, Z_{2}\right)\right)
$$

¿əe

$$
R\left(Z_{1}, Z_{2}\right)=a^{1 / 2+\varepsilon}+\left(\frac{Z_{1}}{a}+\frac{Z_{2}}{a}+1\right)(a, q) a^{\varepsilon} .
$$

Доказательство см. в [24; теорема 2].

СлЕДСТВИЕ 7.5. Предположим, что выполняются условия предложения 7.4 и при $(x, y) \in I$ значения функиии $f(x, y)$ содержатся в отрезке $\left[Z_{3}, Z_{3}+V\right]$, где $Z_{3}>0 u \mathrm{~V} \ll Z_{3}$. Тогда

$$
\begin{aligned}
& \sum_{(x, y) \in I} \sum_{z}[a z-x y=q,(a, x, y, z)=1] f(x, y) \\
& \quad=\frac{K_{a}^{\times}(q)}{a^{2}} \int_{I} f(x, y) d x d y+O\left(a^{-1+\varepsilon} Z_{1} Z_{2} V\right)+O\left(Z_{3} R\left(Z_{1}, Z_{2}\right)\right),
\end{aligned}
$$

где $R\left(Z_{1}, Z_{2}\right)$ определено равенством (7.5).

ДокАЗАтельство. Заменяя в данной сумме $f(x, y)$ на $Z_{3}$ и $Z_{3}+V$, получаем соответственно нижнюю и верхнюю оценки на данную сумму. Применяя предложение 7.4, оценки (6.20) и

$$
\int_{Y_{1}}^{Y_{1}+Z_{1}} d x \int_{Y_{2}}^{Y_{2}+Z_{2}} d y\left(f(x, y)-\left(Z_{3}+\theta V\right)\right) \ll Z_{1} Z_{2} V \quad(\theta=0,1),
$$

приходим к утверждению следствия.

СлеДСТвИЕ 7.6. Предположим, что выполняются условия предложения 7.4 u при $(x, y) \in I$ значения функиии $f(x, y)$ содержатся в отрезке $\left[0, Z_{3}\right]$. Тогда

$$
\begin{gathered}
\sum_{(x, y) \in I} \sum_{z}[a z-x y=q,(a, x, y, z)=1] f(x, y) \\
\ll a^{-1+\varepsilon} Z_{1} Z_{2} Z_{3}+Z_{3} R\left(Z_{1}, Z_{2}\right) .
\end{gathered}
$$

ПРЕДЛОЖЕНИЕ 7.7. Для любъх натуральнъх $r_{1}, r_{2} \leqslant a$

$$
\begin{aligned}
\mathscr{N}_{\ell}(a, q, P)=\varphi^{2}(P) \frac{\varphi(q)}{q^{2}} \frac{K_{a}^{\times}(q)}{a^{2}} \int_{\mathbb{R}^{6}} \frac{\left[X^{\prime \prime \prime} \in \mathscr{M}_{\ell}^{\prime \prime \prime}(a, q, P)\right]}{\left(\operatorname{det} X^{\prime \prime \prime}\right)^{3}} d \boldsymbol{\alpha} d \boldsymbol{\beta} d \boldsymbol{\gamma} \\
+O\left(R_{2}(a, q, P)\right),
\end{aligned}
$$

¿əe

$$
R_{2}(a, q, P)=r_{1}^{3} \sum_{a_{2}, b_{1}} R_{A}\left(\frac{a}{r_{1}}, \frac{b}{r_{1}}, \frac{c}{r_{1}}, c\right)+\frac{P^{2+\varepsilon}}{r_{1} a q}+r_{2}^{2} \frac{P^{2}}{q^{2}} R\left(\frac{a}{r_{2}}, \frac{b}{r_{2}}\right)+\frac{P^{2+\varepsilon}}{r_{2} a q},
$$

а остатки $R\left(Z_{1}, Z_{2}\right), R_{A}\left(Z_{1}, Z_{2}, Z_{3}, Z_{4}\right)$ определяются равенствами (7.5), (7.3) соответственно. 
ЗАмечАниЕ 7.8. Для параметра $\lambda_{2}\left(A^{T}\right)$, определенного в (7.1), будем использовать тривиальную оценку $\lambda_{2}\left(A^{T}\right) \ll q / a$. Использование аналогичной оценки для $\lambda_{2}(A)$ не позволяет выделить главный член в теореме 3.3 . В дальнейшем будет доказано (см. п. 9.1), что в среднем по $A$ выполняется верхняя оценка $\lambda_{2}(A) \leqslant q^{1 / 2+\varepsilon}$, близкая к нижней. Этим обусловлена необходимость сохранения суммирования по $a_{2}$ и $b_{1}$ в остаточном члене $R_{2}(a, q, P)$. Аналогичная ситуация возникнет в предложении 8.3.

ДоКАЗАТЕЛЬСТво ПРЕДЛОЖЕНИЯ 7.7. Переход от (7.10) к (7.11) будет осуществляться в два этапа. Сначала с помощью следствия 7.3 будет сделан переход от суммирования к интегрированию по переменным $a_{3}, b_{3}, c_{1}, c_{2}$, затем с помощью следствия 7.5 - переход от суммирования к интегрированию по переменным $a_{2}, b_{1}$.

Интервалы (их длины оценивались в лемме 5.6)

$$
I_{\nu}=\left[Y_{\nu}, Y_{\nu}+Z_{\nu}\right) \quad\left(\nu=1,2,3 ; Z_{1}=a+1, Z_{2}=\frac{4 q}{a}+1, Z_{3}=\frac{4 P}{q}+1\right),
$$

внутри которых соответственно меняются переменные $a_{3}, b_{3}, c_{1}$, разобьем на $r_{1}$ равных интервалов, а интервалы $I_{\nu}=\left[Y_{\nu}, Y_{\nu}+Z_{\nu}\right)\left(\nu=4,5 ; Z_{4}=a+1\right.$, $Z_{5}=4 q(a+1)$, внутри которых меняются $a_{2}, b_{1}$, на $r_{2}$ равных интервалов:

$$
\begin{array}{ccc}
I_{\nu}=\bigsqcup_{j=0}^{r_{1}-1} I_{\nu}(j), \quad I_{\nu}(j)=\left[Y_{\nu}+\frac{j}{r_{1}} Z_{\nu}, Y_{\nu}+\frac{j+1}{r_{1}} Z_{\nu}\right) & (\nu=1,2,3) ; \\
I_{\nu}=\bigsqcup_{j=0}^{r_{2}-1} I_{\nu}(j), \quad I_{\nu}(j)=\left[Y_{\nu}+\frac{j}{r_{2}} Z_{\nu}, Y_{\nu}+\frac{j+1}{r_{2}} Z_{\nu}\right) & (\nu=4,5) .
\end{array}
$$

Определим функцию

$$
H\left(j_{1}, j_{2} ; b_{1}\right)=\frac{r_{1}}{q} \inf _{\left(a_{3}, b_{3}\right) \in I_{1}\left(j_{1}\right) \times I_{2}\left(j_{2}\right)}\left|a_{1} b_{3}-a_{3} b_{1}\right| \quad\left(0 \leqslant j_{1}, j_{2}<r_{1}\right) .
$$

Из оценок $0 \leqslant H\left(j_{1}, j_{2} ; b_{1}\right) \ll a$ следует, что прямоугольник $I_{1} \times I_{2}$ можно представить в виде $I_{1} \times I_{2}=\bigsqcup_{k=0}^{m} W_{k}\left(b_{1}\right)$, где $m \ll \log a$ и

$$
\begin{aligned}
& W_{k}\left(b_{1}\right)=\bigsqcup_{\substack{j_{1}, j_{2}=0 \\
H\left(j_{1}, j_{2} ; b_{1}\right) \in\left(2^{k-1}, 2^{k}\right]}}^{r_{1}-1} I_{1}\left(j_{1}\right) \times I_{2}\left(j_{2}\right) \quad(k>0), \\
& W_{0}\left(b_{1}\right)=\bigsqcup_{\substack{\left.j_{1}, j_{2}=0 \\
j_{1}, j_{2} ; b_{1}\right) \in[0,1]}}^{r_{1}-1} I_{1}\left(j_{1}\right) \times I_{2}\left(j_{2}\right) .
\end{aligned}
$$

Данную сумму $\mathscr{N}_{\ell}(a, q, P)$ соответственно запишем в виде

$$
\mathscr{N}_{\ell}(a, q, P)=\sum_{k=0}^{m} \mathscr{N}_{\ell, k}(a, q, P),
$$

где

$$
\mathscr{N}_{\ell, k}(a, q, P)=\sum_{a_{2}, b_{1}} \sum_{\left(a_{3}, b_{3}\right) \in W_{k}\left(b_{1}\right)} \sum_{c_{1}, c_{2}}^{\#}\left[X \in \mathscr{M}_{\ell}(a, q, P)\right]
$$


Тогда предложение 7.7 будет доказано, если мы установим справедливость равенства

$$
\mathscr{N}_{\ell, k}(a, q, P)=\frac{\varphi^{2}(P)}{P^{2}} \frac{\varphi(q)}{q^{2}} \frac{K_{a}^{\times}(q)}{a^{2}} \int_{\mathbb{R}^{2}} F_{k}\left(a_{2}, b_{1}\right) d a_{2} d b_{1}+O\left(R_{2}(a, q, P)\right),
$$

где

$$
F_{k}\left(a_{2}, b_{1}\right)=\int_{W_{k}\left(b_{1}\right)} d a_{3} d b_{3} \int_{\mathbb{R}^{2}}\left[X \in \mathscr{M}_{\ell}(a, q, P)\right] d c_{1} d c_{2} .
$$

Действительно, из (7.11) и (7.9) следует, что

$$
\begin{gathered}
\mathscr{N}_{\ell}(a, q, P)=\frac{\varphi^{2}(P)}{P^{2}} \frac{\varphi(q)}{q^{2}} \frac{K_{a}^{\times}(q)}{a^{2}} \int_{\mathbb{R}^{6}}\left[X \in \mathscr{M}_{\ell}(a, q, P)\right] d a_{2} d a_{3} d b_{1} d b_{3} d c_{1} d c_{2} \\
+O\left(R_{2}(a, q, P)\right) .
\end{gathered}
$$

Таким образом, для получения равенства (7.6) остается перейти от переменных $a_{2}, a_{3}, b_{1}, b_{3}, c_{1}, c_{2}$ к переменным $\alpha_{2}, \alpha_{3}, \beta_{1}, \beta_{3}, \gamma_{1}, \gamma_{2}$, воспользовавшись соотношениями (6.21), (6.16) и

$$
\frac{d a_{2} d a_{3}}{\left(\operatorname{det} X^{\prime \prime}\right)^{3}}=\frac{d \alpha_{2} d \alpha_{3}}{a\left(\operatorname{det} X^{\prime \prime \prime}\right)^{3}}
$$

Оценим число прямоугольников разбиения

$$
I_{1} \times I_{2}=\bigsqcup_{j_{1}, j_{2}=0}^{r_{1}-1} I_{1}\left(j_{1}\right) \times I_{2}\left(j_{2}\right)
$$

в каждом из множеств $W_{k}\left(b_{1}\right)$. Если $I_{1}\left(j_{1}\right) \times I_{2}\left(j_{2}\right) \subset W_{k}\left(b_{1}\right)$, то найдется точка $\left(a_{3}, b_{3}\right) \in I_{1}\left(j_{1}\right) \times I_{2}\left(j_{2}\right)$, для которой будет выполняться неравенство $\left|a_{1} b_{3}-a_{3} b_{1}\right| \leqslant 2^{k+1} q / r_{1}$. Так как $a_{1}=a$, то это неравенство на плоскости $O a_{3} b_{3}$ задает полосу ширины $2^{k+2} q /\left(a r_{1}\right) \ll 2^{k} b / r_{1}$ (в направлении оси $\left.O b_{3}\right)$. Значит, над каждой точкой оси $O a_{3}$ лежит $O\left(2^{k}\right)$ прямоугольников разбиения (7.13), имеющих с этой полосой общие точки. Следовательно, множество $W_{k}\left(b_{1}\right)$ состоит из $O\left(r_{1} \cdot 2^{k}\right)$ прямоугольников этого разбиения.

Представим сумму $\mathscr{N}_{\ell, k}(a, q, P)$ в виде

$$
\mathscr{N}_{\ell, k}(a, q, P)=\sum_{a_{2}, b_{1}} G_{k}\left(a_{2}, b_{1}\right)
$$

где

$$
G_{k}\left(a_{2}, b_{1}\right)=\sum_{\left(a_{3}, b_{3}\right) \in W_{k}\left(b_{1}\right)} \sum_{c_{1}, c_{2}}^{\#}\left[X \in \mathscr{M}_{\ell}(a, q, P)\right] .
$$

Докажем асимптотическую формулу

$$
\begin{aligned}
G_{k}\left(a_{2}, b_{1}\right)= & \frac{\varphi^{2}(P)}{P^{2}} \frac{\varphi(q)}{q^{2}} F_{k}\left(a_{2}, b_{1}\right) \\
& +O\left(r_{1}^{3} R_{A}\left(\frac{a}{r_{1}}, \frac{b}{r_{1}}, \frac{c}{r_{1}}, c\right)\right)+O\left(\frac{P^{2+\varepsilon}}{r_{1} q^{2}}\right),
\end{aligned}
$$

где $F_{k}\left(a_{2}, b_{1}\right)$ определено равенством (7.12). 
Отметим, что число точек в каждом из параллелепипедов разбиения (7.13) можно оценить как $O\left(q r_{1}^{-2}\right)$. Поэтому из равенства (6.10) (см. следствие 6.5) следует, что для любого прямоугольника $I_{1}\left(j_{1}\right) \times I_{2}\left(j_{2}\right)$ можно пользоваться следующей формулой с тривиальной оценкой остаточного члена:

$$
\begin{aligned}
& \sum_{\left(a_{3}, b_{3}\right) \in I_{1}\left(j_{1}\right) \times I_{2}\left(j_{2}\right)} \sum_{c_{1}, c_{2}}^{\#}\left[X \in \mathscr{M}_{\ell}(a, q, P)\right]=\frac{\varphi^{2}(P)}{P^{2}} \frac{\varphi(q)}{q^{2}} \\
& \times \int_{I_{1}\left(j_{1}\right) \times I_{2}\left(j_{2}\right)} d a_{3} d b_{3} \int_{\mathbb{R}^{2}}\left[X \in \mathscr{M}_{\ell}(a, q, P)\right] d c_{1} d c_{2}+O\left(\frac{P^{2}}{q^{2} r_{1}^{2}}\right) .
\end{aligned}
$$

При $k=0$ равенство (7.15) следует из (7.16). Поэтому будем в дальнейшем предполагать, что $k>0$.

При фиксированных $a_{2}, b_{1}$ по определению матриц Минковского (3.3), (3.4) пара переменных $\left(a_{3}, b_{3}\right)$ может меняться внутри некоторого прямоугольника П $\left(a_{2}, b_{1}\right)$. Обозначим через $\Omega_{1,2}\left(a_{2}, b_{1}\right)$ область, состоящую из тех прямоугольников разбиения (7.13), которые полностью содержатся в $\Pi\left(a_{2}, b_{1}\right)$, а через $\Omega_{1,2}^{\prime}\left(a_{2}, b_{1}\right)$ - область, состоящую из прямоугольников разбиения $(7.13)$, имеющих общие точки с границей $\Pi\left(a_{2}, b_{1}\right)$. Очевидно, что $\Omega_{1,2}^{\prime}\left(a_{2}, b_{1}\right)$ состоит из $O\left(r_{1}\right)$ прямоугольников разбиения (7.13), и по формуле $(7.16)$

$$
\begin{aligned}
& \sum_{\left(a_{3}, b_{3}\right) \in \Omega_{1,2}^{\prime}\left(a_{2}, b_{1}\right)} \sum_{c_{1}, c_{2}}^{\#}\left[X \in \mathscr{M}_{\ell}(a, q, P)\right] \\
& =\frac{\varphi^{2}(P)}{P^{2}} \frac{\varphi(q)}{q^{2}} \int_{\Omega_{1,2}^{\prime}\left(a_{2}, b_{1}\right)} d a_{3} d b_{3} \int_{\mathbb{R}^{2}}\left[X \in \mathscr{M}_{\ell}(a, q, P)\right] d c_{1} d c_{2}+O\left(\frac{P^{2+\varepsilon}}{q^{2} r_{1}}\right) .
\end{aligned}
$$

Поэтому для доказательства равенства (7.15) достаточно проверить, что

$$
\begin{aligned}
& \sum_{\left(a_{3}, b_{3}\right) \in \widetilde{W}_{k}\left(a_{2}, b_{1}\right)} \sum_{c_{1}, c_{2}}^{\#}\left[X \in \mathscr{M}_{\ell}(a, q, P)\right] \\
& =\frac{\varphi^{2}(P)}{P^{2}} \frac{\varphi(q)}{q^{2}} \int_{\widetilde{W}_{k}\left(a_{2}, b_{1}\right)} d a_{3} d b_{3} \int_{\mathbb{R}^{2}}\left[X \in \mathscr{M}_{\ell}(a, q, P)\right] d c_{1} d c_{2} \\
& \quad+O\left(r_{1}^{3} R_{A}\left(\frac{a}{r_{1}}, \frac{b}{r_{1}}, \frac{c}{r_{1}}, c\right)\right)+O\left(\frac{P^{2+\varepsilon}}{q^{2} r_{1}}\right),
\end{aligned}
$$

где $\widetilde{W}_{k}\left(a_{2}, b_{1}\right)=W_{k}\left(b_{1}\right) \cap \Omega_{1,2}\left(a_{2}, b_{1}\right)$.

Зафиксируем произвольный прямоугольник $I_{1}\left(j_{1}\right) \times I_{2}\left(j_{2}\right) \subset \widetilde{W}_{k}\left(a_{2}, b_{1}\right)$. На каждом из параллелепипедов $I_{1}\left(j_{1}\right) \times I_{2}\left(j_{2}\right) \times I_{3}\left(j_{3}\right)$ применим следствие 7.3 . Так как $\left(a_{3}, b_{3}\right) \in W_{k}\left(b_{1}\right)(k>0)$, то $\left|a_{1} b_{3}-a_{3} b_{1}\right| \gg U=2^{k} q / r_{1}$, и по лемме 5.7 для каждой из функций $f_{i}$, задающих границу множества $\Omega(\bar{A})$, выполняются оценки

$$
\frac{\partial f_{i}}{\partial a_{3}} \ll \frac{r_{1}}{2^{k}} \frac{P}{a q}, \quad \frac{\partial f_{i}}{\partial b_{3}} \ll \frac{r_{1}}{2^{k}} \frac{P}{b q}, \quad \frac{\partial f_{i}}{\partial c_{1}} \ll \frac{r_{1}}{2^{k}} \frac{P}{c q} .
$$

Значит, на каждом из параллелепипедов $I_{1}\left(j_{1}\right) \times I_{2}\left(j_{2}\right) \times I_{3}\left(j_{3}\right)$ значения функции $f_{i}$ (при условии, что ее график задает границу) содержатся в интервале 
длины $V=O\left(P \cdot 2^{-k} q^{-1}\right)$. Согласно следствию 7.3

$$
\begin{gathered}
\sum_{\left(a_{3}, b_{3}, c_{1}\right) \in I_{1}\left(j_{1}\right) \times I_{2}\left(j_{2}\right) \times I_{3}\left(j_{3}\right)} \sum_{c_{2}}\left[X \in \mathscr{M}_{\ell}(a, q, P)\right] \\
=\frac{\varphi^{2}(P)}{P^{2}} \frac{\varphi(q)}{q^{2}} \int_{I_{1}\left(j_{1}\right) \times I_{2}\left(j_{2}\right) \times I_{3}\left(j_{3}\right)} d a_{3} d b_{3} d c_{1} \int_{\mathbb{R}} d c_{2}\left[X \in \mathscr{M}_{\ell}(a, q, P)\right] \\
+O\left(R_{A}\left(\frac{a}{r_{1}}, \frac{b}{r_{1}}, \frac{c}{r_{1}}, c\right)\right)+O\left(\frac{P^{2}}{r_{1}^{3} q^{2} \cdot 2^{k}}\right) .
\end{gathered}
$$

Суммируя последнее равенство по $j_{3}$ и по прямоугольникам $I_{1}\left(j_{1}\right) \times I_{2}\left(j_{2}\right) \subset$ $\widetilde{W}_{k}\left(a_{2}, b_{1}\right)$ (число которых есть $\left.O\left(r_{1} \cdot 2^{k}\right)\right)$, приходим к равенству (7.17). Таким образом, равенство (7.15) тоже доказано.

Подставляя (7.15) в (7.14), получаем, что для доказательства основной формулы (7.6) достаточно убедиться в справедливости равенства

$$
\begin{array}{r}
\sum_{a_{2}, b_{1}}^{\times} F_{k}\left(a_{2}, b_{1}\right)=\frac{K_{a}^{\times}(q)}{a^{2}} \int_{\mathbb{R}^{2}} F_{k}\left(a_{2}, b_{1}\right) d a_{2} d b_{1}+O\left(\frac{P^{2+\varepsilon}}{a r_{1}}\right) \\
+O\left(\frac{P^{2+\varepsilon}}{a r_{2}}\right)+O\left(r_{2}^{2} \frac{P^{2}}{q} R\left(\frac{a}{r_{2}}, \frac{b}{r_{2}}\right)\right) .
\end{array}
$$

Отметим, что

$$
\int_{\mathbb{R}^{2}}\left[X \in \mathscr{M}_{\ell}(a, q, P)\right] d c_{1} d c_{2} \ll \frac{P^{2}}{q^{2}},
$$

поэтому для функции $F_{k}$ всегда выполняется тривиальная оценка

$$
F_{k}\left(a_{2}, b_{1}\right) \ll \frac{2^{k}}{r_{1}} \frac{P^{2}}{q} .
$$

Поэтому, согласно следствию 7.6, в каждом из прямоугольников разбиения

$$
I_{4} \times I_{5}=\bigsqcup_{j_{4}, j_{5}=0}^{r_{2}-1} I_{4}\left(j_{4}\right) \times I_{5}\left(j_{5}\right)
$$

можно применять формулу

$$
\begin{aligned}
\sum_{\left(a_{2}, b_{1}\right) \in I_{4}\left(j_{4}\right) \times I_{5}\left(j_{5}\right)}^{\times} F_{k}\left(a_{2}, b_{1}\right)=\frac{K_{a}^{\times}(q)}{a^{2}} \int_{I_{4}\left(j_{4}\right) \times I_{5}\left(j_{5}\right)} F_{k}\left(a_{2}, b_{1}\right) d a_{2} d b_{1} \\
+O\left(\frac{P^{2+\varepsilon}}{a r_{2}^{2}}\right)+O\left(\frac{P^{2}}{q} R\left(\frac{a}{r_{2}}, \frac{b}{r_{2}}\right)\right) .
\end{aligned}
$$

Граница области изменения пары переменных $\left(a_{2}, b_{1}\right)$ определяется условиями $b_{2} \geqslant a_{1}$ и $b_{2} \geqslant\left|b_{1}\right|$ (где $\left.b_{2}=\left(q+a_{2} b_{1}\right) / a_{1}\right)$ и поэтому состоит из участков графиков функций

$$
b_{1}\left(a_{2}\right)=\frac{q-a_{1}^{2}}{a_{2}}, \quad b_{1}\left(a_{2}\right)=\frac{q}{ \pm a_{1}-a_{2}} .
$$


Обозначим через $\Omega_{4,5}$ область, состоящую из тех прямоугольников разбиения (7.22), которые полностью содержатся в области изменения $\left(a_{2}, b_{1}\right)$, а через $\Omega_{4,5}^{\prime}$ - область, состоящую из тех прямоугольников разбиения $(7.22)$, которые пересекают границу области изменения $\left(a_{2}, b_{1}\right)$. Из монотонности функций (7.24) следует, что $\Omega_{4,5}^{\prime}$ состоит из $O\left(r_{2}\right)$ прямоугольников разбиения $(7.22)$. Применяя в каждом из них формулу (7.23), получаем равенство

$$
\begin{aligned}
\sum_{\left(a_{2}, b_{1}\right) \in \Omega_{4,5}^{\prime}}^{\times} F_{k}\left(a_{2}, b_{1}\right)=\frac{K_{a}^{\times}(q)}{a^{2}} \int_{\Omega_{4,5}^{\prime}} F_{k}\left(a_{2}, b_{1}\right) d a_{2} d b_{1} \\
+O\left(\frac{P^{2+\varepsilon}}{a r_{2}}\right)+O\left(r_{2} \frac{P^{2}}{q} R\left(\frac{a}{r_{2}}, \frac{b}{r_{2}}\right)\right) .
\end{aligned}
$$

Поэтому для доказательства равенства (7.19) достаточно проверить справедливость асимптотической формулы

$$
\begin{aligned}
\sum_{\left(a_{2}, b_{1}\right) \in \Omega_{4,5}}^{\times} F_{k}\left(a_{2}, b_{1}\right)= & \frac{K_{a}^{\times}(q)}{a^{2}} \int_{\Omega_{4,5}} F_{k}\left(a_{2}, b_{1}\right) d a_{2} d b_{1} \\
& +O\left(\frac{P^{2+\varepsilon}}{a r_{1}}\right)+O\left(\frac{P^{2+\varepsilon}}{a r_{2}}\right)+O\left(r_{2}^{2} \frac{P^{2}}{q} R\left(\frac{a}{r_{2}}, \frac{b}{r_{2}}\right)\right) .
\end{aligned}
$$

Это равенство в свою очередь является следствием того, что для любого прямоугольника $I_{4}\left(j_{4}\right) \times I_{5}\left(j_{5}\right) \subset \Omega_{4,5}$

$$
\begin{gathered}
\sum_{\left(a_{2}, b_{1}\right) \in I_{4}\left(j_{4}\right) \times I_{5}\left(j_{5}\right)}^{\times} F_{k}\left(a_{2}, b_{1}\right)=\frac{K_{a}^{\times}(q)}{a^{2}} \int_{I_{4}\left(j_{4}\right) \times I_{5}\left(j_{5}\right)} F_{k}\left(a_{2}, b_{1}\right) d a_{2} d b_{1} \\
+O\left(\frac{P^{2+\varepsilon}}{a r_{1} r_{2}^{2}}\right)+O\left(\frac{P^{2+\varepsilon}}{a r_{2}^{3}}\right)+O\left(\frac{P^{2}}{q} R\left(\frac{a}{r_{2}}, \frac{b}{r_{2}}\right)\right) .
\end{gathered}
$$

Последнее соотношение вытекает из следствия 7.5 при условии, что значения $F_{k}\left(a_{2}, b_{1}\right)$ меняются внутри интервала длины

$$
V \ll \frac{P^{2}}{q}\left(\frac{1}{r_{1}}+\frac{1}{r_{2}}\right) .
$$

Проверим справедливость этого условия.

Так как $\left(a_{3}, b_{3}\right) \in W_{k}\left(b_{1}\right)$, то по лемме 5.7 каждая из функций $f_{i}$, задающих границу области $\Omega(\bar{A})$ (при $\left.b_{2}=\left(q-a_{2} b_{1}\right) a_{1}^{-1}\right)$, удовлетворяет оценкам, аналогичным оценкам (7.18):

$$
\frac{\partial f_{i}}{\partial a_{2}} \ll \frac{r_{1}}{2^{k}} \frac{P}{a q}, \quad \frac{\partial f_{i}}{\partial b_{1}} \ll \frac{r_{1}}{2^{k}} \frac{P}{b q} .
$$

Поэтому при $\left(a_{2}, b_{1}\right) \in I_{4}\left(j_{4}\right) \times I_{5}\left(j_{5}\right)$ площадь области $\Omega(\bar{A})$ может меняться внутри интервала длины $O\left(\frac{r_{1}}{2^{k}} \frac{P^{2}}{r_{2} q^{2}}\right)$. Это приводит к тому, что значения $F_{k}\left(a_{2}, b_{1}\right)$ могут меняться в интервале длины

$$
O\left(\operatorname{Area}\left(W_{k}\left(b_{1}\right)\right) \frac{r_{1}}{2^{k}} \frac{P^{2}}{r_{2} q^{2}}\right)=O\left(\frac{P^{2}}{r_{2} q}\right),
$$

что согласуется с оценкой (7.25). 
Значения $F_{k}\left(a_{2}, b_{1}\right)$ могут меняться и из-за того, что меняется область $W_{k}$. Пусть разным значениям $b_{1}$ соответствуют разные области $W_{k}\left(b_{1}\right)$. Предположим, что $b_{1}, b_{1}^{\prime} \in I_{5}\left(j_{5}\right)$ и $W_{k}\left(b_{1}\right) \neq W_{k}\left(b_{1}^{\prime}\right)$. Пусть, например, $I_{1}\left(j_{1}\right) \times I_{2}\left(j_{2}\right) \subset$ $W_{k}\left(b_{1}\right) \backslash W_{k}\left(b_{1}^{\prime}\right)$, т. е.

$$
H\left(j_{1}, j_{2} ; b_{1}\right) \in\left(2^{k-1}, 2^{k}\right], \quad H\left(j_{1}, j_{2} ; b_{1}^{\prime}\right) \notin\left(2^{k-1}, 2^{k}\right],
$$

где функция $H$ определена равенством (7.8). Значения функции $H$ при фиксированных $j_{1}, j_{2}$ и разных $b_{1}, b_{1}^{\prime}$ могут отличаться на $O\left(r_{1} / r_{2}\right)$. Kроме того, $H\left(j_{1}, j_{2}+1 ; b_{1}\right)-H\left(j_{1}, j_{2} ; b_{1}\right) \gg 1$. Поэтому при фиксированном $j_{1}$ число номеров $j_{2}$, для которых могут выполняться условия (7.26), можно оценить как $O\left(r_{1} / r_{2}+1\right)$. Таким образом, площади фигур $W_{k}\left(b_{1}\right)$ и $W_{k}\left(b_{1}^{\prime}\right)$ отличаются не более чем на

$$
O\left(\left(\frac{r_{1}}{r_{2}}+1\right) r_{1} \frac{q}{r_{1}^{2}}\right)=O\left(q\left(\frac{1}{r_{1}}+\frac{1}{r_{2}}\right)\right)
$$

Отсюда и из оценки (7.20) следует, что значения $F_{k}\left(a_{2}, b_{1}\right)$ при этом могут меняться в интервале длины

$$
O\left(\frac{P^{2}}{q^{2}} q\left(\frac{1}{r_{1}}+\frac{1}{r_{2}}\right)\right)=O\left(\frac{P^{2}}{q}\left(\frac{1}{r_{1}}+\frac{1}{r_{2}}\right)\right)
$$

что снова согласуется с оценкой (7.25).

\section{8. Третий вариант оценки остатка}

Теорема 8.1. Пусть матрица $A=\left(\begin{array}{ll}a_{1} & a_{2} \\ b_{1} & b_{2}\end{array}\right)$ и коэффициент а ваньи, $q=\operatorname{det} A \neq 0,\left(a_{1}, a_{2}, b_{1}, b_{2}\right)=1, D=\left(a_{1}, a_{2}\right),\left(D, a_{3}\right)=1$, и матрица $X$ имеет вид (1.8). Тогда для любого прямоугольника

$$
I=\left[Y_{2}, Y_{2}+Z_{2}\right) \times\left[Y_{3}, Y_{3}+Z_{3}\right) \times\left[Y_{4}, Y_{4}+Z_{4}\right),
$$

размеры которого удовлетворяют условиям $1 \leqslant Z_{2} \leqslant q, 1 \leqslant Z_{3}, Z_{4} \leqslant P q$, выполняется равенство

$$
\sum_{\left(b_{3}, c_{1}, c_{2}\right) \in I} \sum_{c_{3}}^{\#}[\operatorname{det} X=P]=\frac{\varphi^{2}(P)}{P^{2}} \frac{\varphi(q)}{q^{2}} \frac{D}{\varphi(D)} Z_{2} Z_{3} Z_{4}+O\left(R_{A}\left(Z_{2}, Z_{3}, Z_{4}\right)\right),
$$

əдe

$$
\begin{aligned}
R_{A}\left(Z_{2}, Z_{3}, Z_{4}\right)=( & P, q)(P q)^{\varepsilon}\left(\frac{Z_{2} Z_{3} Z_{4}}{q}\left(\frac{1}{Z_{2}}+\frac{1}{Z_{3}}+\frac{1}{Z_{4}}\right) D\right. \\
& \left.+q^{1 / 2}+\lambda_{2}^{1 / 2}(A)\left(Z_{3}+Z_{4}\right)\right)
\end{aligned}
$$

и $\lambda_{2}(A)$ определено в (7.1).

Доказательство см. в [23]. 
СлЕДСТвиЕ 8.2. Пусть выполнены условия теоремы 8.1, размеры прямоугольника

$$
I^{\prime}=\left[Y_{2}, Y_{2}+Z_{2}\right) \times\left[Y_{3}, Y_{3}+Z_{3}\right)
$$

удовлетворяют неравенствам $1 \leqslant Z_{2} \leqslant q, 1 \leqslant Z_{3} \leqslant P q$ и при $\left(b_{3}, c_{1}\right) \in I^{\prime}$ значения неотрицательной функиии $f\left(b_{3}, c_{1}\right)$ меняются внутри интервала $\left[Z_{4}, Z_{4}+V\right)$, где $1 \leqslant V \ll Z_{4} \leqslant P q$. Тогда

$$
\begin{array}{r}
\sum_{\left(b_{3}, c_{1}\right) \in I^{\prime}} \sum_{0<c_{2} \leqslant f\left(b_{3}, c_{1}\right)} \sum_{c_{3}}^{\#}[\operatorname{det} X=P]=\frac{\varphi^{2}(P)}{P^{2}} \frac{\varphi(q)}{q^{2}} \frac{D}{\varphi(D)} \int_{I^{\prime}} f\left(b_{3}, c_{1}\right) d b_{3} d c_{1} \\
+O\left(R_{A}\left(Z_{2}, Z_{3}, Z_{4}\right)\right)+O\left(\frac{Z_{2} Z_{3} V}{q}\right) .
\end{array}
$$

Доказательство аналогично доказательству следствия 7.3.

ПРЕДЛОЖЕНИЕ 8.3. Пусть $r$ - натуральное число $u 1 \leqslant r \leqslant a$. Тогда для суммы $\mathscr{N}_{\ell}(a, q, P)$, задаваемой равенством (5.7), справедлива асимптотическая формула

$$
\begin{aligned}
\mathscr{N}_{\ell}(a, q, P)=\varphi^{2}(P) & \left(\frac{\varphi(q)}{q^{2}} \int_{\mathbb{R}^{4}} d \boldsymbol{\beta} d \boldsymbol{\gamma} \sum_{a_{2}, a_{3}}^{\#} c(q, D) \frac{\left[X^{\prime \prime} \in \mathscr{M}_{\ell}^{\prime \prime}(a, q, P)\right]}{\left(\operatorname{det} X^{\prime \prime}\right)^{3}}+\rho_{3}(a, q)\right) \\
& +O\left(R_{3}(a, q, P)\right),
\end{aligned}
$$

где $c(q, D)$ определено равенством $(6.18), \rho_{3}(a, q) \ll a q^{-2+\varepsilon}$,

$$
R_{3}(a, q, P)=r^{2} a \sum_{a_{2}, b_{1}} R_{A}\left(\frac{b}{r}, \frac{c}{r}, c\right)+\frac{P^{2+\varepsilon}}{r a q}
$$

и $R_{A}\left(Z_{2}, Z_{3}, Z_{4}\right)$ - остаток из теоремъ 8.1.

ДокАзАТЕльство. Первая часть доказательства по сути повторяет доказательство предложения 7.7.

Интервалы $I_{2}=\left[Y_{2}, Y_{2}+Z_{2}\right), I_{3}=\left[Y_{3}, Y_{3}+Z_{3}\right)$ (см. (7.7)), внутри которых соответственно меняются переменные $b_{3}, c_{1}$, разобьем на $r$ равных интервалов:

$$
I_{\nu}=\bigsqcup_{j=0}^{r-1} I_{\nu}(j), \quad I_{\nu}(j)=\left[Y_{\nu}+\frac{j}{r} Z_{\nu}, Y_{\nu}+\frac{j+1}{r} Z_{\nu}\right) \quad(\nu=2,3)
$$

Определим функцию

$$
H\left(j ; b_{1}\right)=\frac{r}{q} \inf _{b_{3} \in I_{2}(j)}\left|a_{1} b_{3}-a_{3} b_{1}\right| \quad(0 \leqslant j<r) .
$$

Интервал $I_{2}$ представим в виде $I_{2}=\bigsqcup_{k=0}^{m} W_{k}\left(b_{1}\right)$, где $m \ll \log a$,

$$
W_{k}\left(b_{1}\right)=\bigsqcup_{\substack{j=0 \\ H\left(j ; b_{1}\right) \in\left(2^{k-1}, 2^{k}\right]}}^{r-1} I_{2}(j) \quad(k>0), \quad W_{0}\left(b_{1}\right)=\bigsqcup_{\substack{j=0 \\ H\left(j ; b_{1}\right) \in[0,1]}}^{r-1} I_{2}(j)
$$


Данную сумму $\mathscr{N}_{\ell}(a, q, P)$ запишем в виде

$$
\mathscr{N}_{\ell}(a, q, P)=\sum_{k=0}^{m} \mathscr{N}_{\ell, k}(a, q, P),
$$

где

$$
\begin{aligned}
\mathscr{N}_{\ell, k}(a, q, P) & =\sum_{a_{2}, a_{3}, b_{1}} G_{k}\left(a_{2}, a_{3}, b_{1}\right), \\
G_{k}\left(a_{2}, a_{3}, b_{1}\right) & =\sum_{b_{3} \in W_{k}\left(b_{1}\right)} \sum_{c_{1}, c_{2}}^{\#}\left[X \in \mathscr{M}_{\ell}(a, q, P)\right] .
\end{aligned}
$$

Проверим справедливость асимптотической формулы

$$
\begin{gathered}
G_{k}\left(a_{2}, a_{3}, b_{1}\right)=\frac{\varphi^{2}(P)}{P^{2}} \frac{\varphi(q)}{q^{2}} \frac{D}{\varphi(D)} \int_{W_{k}\left(b_{1}\right)} d b_{3} \int_{\mathbb{R}^{2}}\left[X \in \mathscr{M}_{\ell}(a, q, P)\right] d c_{1} d c_{2} \\
+O\left(r \cdot 2^{k} R\left(\frac{b}{r}, \frac{c}{r}, c\right)\right)+O\left(\frac{P^{2+\varepsilon}}{r a q^{2}}\right) .
\end{gathered}
$$

Для этого оценим сначала число интервалов $I_{2}(j)$, содержащихся в каждом из множеств $W_{k}\left(b_{1}\right)$. Если $I_{2}(j) \subset W_{k}\left(b_{1}\right)$, то найдется точка $b_{3} \in I_{2}(j)$, для которой будет выполняться неравенство

$$
\left|a_{1} b_{3}-a_{3} b_{1}\right| \leqslant \frac{2^{k+1} q}{r} .
$$

Так как $a_{1}=a$, то это неравенство на оси $O b_{3}$ задает отрезок длины $2^{k+2} q /(a r)$. Значит, существует $O\left(2^{k}\right)$ интервалов $I_{2}(j)$, которые имеют общие точки с этим отрезком, т. е. множество $W_{k}\left(b_{1}\right)$ состоит из $O\left(2^{k}\right)$ интервалов разбиения.

Из равенства (6.10) следует, что для любого интервала $I_{2}(j)$ выполняется асимптотическая формула с тривиальной оценкой остаточного члена:

$$
\begin{aligned}
& \sum_{b_{3} \in I_{2}(j)} \sum_{c_{1}, c_{2}}^{\#}\left[X \in \mathscr{M}_{\ell}(a, q, P)\right]=\frac{\varphi^{2}(P)}{P^{2}} \frac{\varphi(q)}{q^{2}} \frac{D}{\varphi(D)} \\
& \quad \times \int_{I_{2}(j)} d b_{3} \int_{\mathbb{R}^{2}}\left[X \in \mathscr{M}_{\ell}(a, q, P)\right] d c_{1} d c_{2}+O\left(\frac{P^{2+\varepsilon}}{r a q^{2}}\right) .
\end{aligned}
$$

При $k=0$ равенство (8.5) следует из (8.7). Поэтому будем в дальнейшем предполагать, что $k>0$.

Равенство (8.5) будет доказано, если для любого интервала $I_{2}(j) \subset W_{k}\left(b_{1}\right)$ будет установлена справедливость формулы

$$
\begin{aligned}
& \sum_{b_{3} \in I_{2}(j)} \sum_{c_{1}, c_{2}}^{\#}\left[X \in \mathscr{M}_{\ell}(a, q, P)\right] \\
& =\frac{\varphi^{2}(P)}{P^{2}} \frac{\varphi(q)}{q^{2}} \frac{D}{\varphi(D)} \int_{I_{2}(j)} d b_{3} \int_{\mathbb{R}^{2}}\left[X \in \mathscr{M}_{\ell}(a, q, P)\right] d c_{1} d c_{2} \\
& \quad+O\left(r R\left(\frac{b}{r}, \frac{c}{r}, c\right)\right)+O\left(\frac{P^{2+\varepsilon}}{r \cdot 2^{k} a q^{2}}\right) .
\end{aligned}
$$


(Для интервалов $I_{2}(j)$, которые пересекаются с $W_{k}\left(b_{1}\right)$ лишь частично, достаточно применить (8.7).)

Если $b_{3} \in W_{k}\left(b_{1}\right)$, то $\left|a_{1} b_{3}-a_{3} b_{1}\right| \gg U=2^{k} q / r$. Поэтому из условий $(5.6)$ следует, что для каждой из функций $f_{i}$, графики которых задают границу множества $\Omega(\bar{A})$, выполняются оценки

$$
\frac{\partial f_{i}}{\partial b_{3}} \ll \frac{r}{2^{k}} \frac{P}{b q}, \quad \frac{\partial f_{i}}{\partial c_{1}} \ll \frac{r}{2^{k}} .
$$

Значит на каждом из прямоугольников $I_{2}\left(j_{2}\right) \times I_{3}\left(j_{3}\right)$ значения функций $f_{i}$ (при условии, что соответствующая часть графика задает границу) содержатся в интервале длины $V=O\left(P q^{-1} \cdot 2^{-k}\right)$. На каждом из параллелепипедов $I\left(j_{2}, j_{3}\right)=I_{2}\left(j_{2}\right)$, где $I_{2}\left(j_{2}\right) \subset W_{k}$, применим следствие 8.2:

$$
\begin{aligned}
& \sum_{\left(b_{3}, c_{1}\right) \in I_{2}\left(j_{2}\right) \times I_{3}\left(j_{3}\right)} \sum_{c_{2}}^{\#}\left[X \in \mathscr{M}_{\ell}(a, q, P)\right] \\
& =\frac{\varphi^{2}(P)}{P^{2}} \frac{\varphi(q)}{q^{2}} \frac{D}{\varphi(D)} \int_{I_{2}\left(j_{2}\right) \times I_{3}\left(j_{3}\right)} d b_{3} d c_{1} \int_{\mathbb{R}} d c_{2}\left[X \in \mathscr{M}_{\ell}(P)\right] \\
& \quad+O\left(R\left(\frac{b}{r}, \frac{c}{r}, c\right)\right)+O\left(\frac{P^{2}}{2^{k} r^{2} a q^{2}}\right)
\end{aligned}
$$

Суммируя полученное равенство по $j_{3}$, приходим к (8.8). Тем самым, равенство (8.5) доказано.

Дальнейшие шаги будут отличаться от доказательства предложения 7.7.

Из равенств (8.3)-(8.5) получаем, что

$$
\begin{gathered}
\mathscr{N}_{\ell}(a, q, P)=\frac{\varphi^{2}(P)}{P^{2}} \frac{\varphi(q)}{q^{2}} \sum_{a_{2}, a_{3}, b_{1}}^{\#} \frac{D}{\varphi(D)} \int_{\mathbb{R}^{3}}\left[X \in \mathscr{M}_{\ell}(a, q, P)\right] d b_{3} d c_{1} d c_{2} \\
+O\left(R_{3}(a, q, P)\right) .
\end{gathered}
$$

С помощью равенств (6.21) перейдем к переменным $\gamma_{1}, \gamma_{2}$ :

$$
\begin{aligned}
\mathscr{N}_{\ell}(a, q, P)= & \varphi^{2}(P) \frac{\varphi(q)}{q} \sum_{a_{2}, a_{3}, b_{1}}^{\#} \frac{D}{\varphi(D)} \int_{\mathbb{R}^{2}} d \gamma \int_{\mathbb{R}} \frac{d b_{3}\left[X \in \mathscr{M}_{\ell}(a, q, P)\right]}{\left(\operatorname{det} X^{\prime}\right)^{3}} \\
& \quad+O\left(R_{3}(a, q, P)\right) \\
= & \varphi^{2}(P) \frac{\varphi(q)}{q} \int_{\mathbb{R}^{2}} S(a, q, \gamma) d \boldsymbol{\gamma}+O\left(R_{3}(a, q, P)\right)
\end{aligned}
$$

где

$$
S(a, q, \gamma)=\sum_{a_{2}, a_{3}, b_{1}}^{\#} \frac{D}{\varphi(D)} \int_{\mathbb{R}} \frac{d b_{3}\left[X \in \mathscr{M}_{\ell}(a, q, P)\right]}{\left(\operatorname{det} X^{\prime}\right)^{3}}
$$


Для доказательства предложения остается проверить, что для суммы $S(a, q, \gamma)$ справедлива асимптотическая формула

$$
S(a, q, \gamma)=\frac{1}{q} \int_{\mathbb{R}^{2}} d \boldsymbol{\beta} \sum_{a_{2}, a_{3}}^{\#} c(q, D) \frac{\left[X^{\prime \prime} \in \mathscr{M}_{\ell}^{\prime \prime}(a, q, P)\right]}{\left(\operatorname{det} X^{\prime \prime}\right)^{3}}+O\left(a q^{-2+\varepsilon}\right)
$$

где $c(q, D)$ определено равенством (6.18).

Предположим, что значения $a_{2}, a_{3}, b_{3}, \gamma_{1}$ и $\gamma_{2}$ фиксированы, и рассмотрим сумму

$$
\sigma(I)=\sum_{b_{1} \in I} \frac{\left[\left(a_{1}, a_{2}, b_{1}, b_{2}\right)=1\right]}{\left(\operatorname{det} X^{\prime}\right)^{3}} .
$$

Пусть $\left(u_{0}, v_{0}\right)$ - частное решение уравнения $\left|\begin{array}{cc}a_{1} & a_{2} \\ u & v\end{array}\right|=D$. Тогда

$$
\left|\begin{array}{cc}
a_{1} & a_{2} \\
u_{0} q / d & v_{0} q / d
\end{array}\right|=q
$$

и все решения уравнения $\left|\begin{array}{ll}a_{1} & a_{2} \\ b_{1} & b_{2}\end{array}\right|=q$ относительно неизвестных $b_{1}, b_{2}$ можно записать в виде

$$
\left(\begin{array}{l}
b_{1}(t) \\
b_{2}(t)
\end{array}\right)=\left(\begin{array}{l}
u_{0} q / D+t a_{1} / D \\
v_{0} q / D+t a_{2} / D
\end{array}\right)=\left(\begin{array}{ll}
a_{1} / D & u_{0} \\
a_{2} / D & v_{0}
\end{array}\right)\left(\begin{array}{c}
t \\
q / D
\end{array}\right) \quad(t \in \mathbb{Z}) .
$$

Так как $\left(\begin{array}{ll}a_{1} / D & u_{0} \\ a_{2} / D & v_{0}\end{array}\right) \in S L_{2}(\mathbb{Z})$, то $\left(b_{1}(t), b_{2}(t)\right)=(t, q / D)$ и $\left(a_{1}, a_{2}, b_{1}(t), b_{2}(t)\right)=$ $(D, t, q / D)$. Поэтому

$$
\begin{aligned}
\sigma(I) & =\sum_{t: b_{1}(t) \in I}\left|\begin{array}{ccc}
a_{1} & a_{2} & a_{3} \\
b_{1}(t) & b_{2}(t) & b_{3} \\
\gamma_{1} & \gamma_{2} & 1
\end{array}\right|^{-3}\left[\left(D, t, \frac{q}{D}\right)=1\right] \\
& =\sum_{\delta \mid(D, q / D)} \mu(\delta) \sum_{t: b_{1}(\delta t) \in I} F(t),
\end{aligned}
$$

где

$$
F(t)=\left|\begin{array}{ccc}
a_{1} & a_{2} & a_{3} \\
b_{1}(\delta t) & b_{2}(\delta t) & b_{3} \\
\gamma_{1} & \gamma_{2} & 1
\end{array}\right|^{-3}
$$

Эта функция имеет конечное число участков монотонности и удовлетворяет оценке $F(t) \ll q^{-3}$. По лемме 6.6

$$
\begin{aligned}
\sigma(I) & =\sum_{\delta \mid(D, q / D)} \mu(\delta)\left(\frac{D}{\delta a} \int_{I} \frac{d b_{1}}{\left(\operatorname{det} X^{\prime}\right)^{3}}+O\left(\frac{1}{q^{3}}\right)\right) \\
& =\frac{D}{a} \frac{\varphi((D, q / D))}{(D, q / D)} \int_{I} \frac{d b_{1}}{\left(\operatorname{det} X^{\prime}\right)^{3}}+O\left(q^{-3+\varepsilon}\right) .
\end{aligned}
$$


Полученная формула для $\sigma(I)$ позволяет в равенстве (8.9) заменить суммирование по переменной $b_{1}$ интегрированием. Пользуясь определением величины $c(q, D)(6.18)$, находим, что

$$
S(a, q, \gamma)=\sum_{a_{2}, a_{3}}^{\#} \frac{c(q, D)}{a} \int_{\mathbb{R}^{2}} \frac{d b_{1} d b_{3}\left[X^{\prime} \in \mathscr{M}_{\ell}^{\prime}(a, q, P)\right]}{\left(\operatorname{det} X^{\prime}\right)^{3}}+O\left(a q^{-2+\varepsilon}\right) .
$$

Переходя от переменных $b_{1}, b_{3}$ к переменным $\beta_{1}, \beta_{3}$ и пользуясь равенствами (6.16), получаем нужную формулу (8.10). Предложение 8.3 доказано.

\section{9. Завершение доказательства основного результата}

\section{1. О приведенных базисах в плоских решетках.}

Лемма 9.1. Пусть $Q$ - натуральное число, $a_{1}, \ldots, a_{Q}$ и $\lambda_{1} \leqslant \cdots \leqslant \lambda_{Q}-$ действительные числа, а $g$ - непрерывно дифференцируемая функция, заданная на отрезке $\left[\lambda_{1}, \lambda_{Q}\right]$. Тогда

$$
\sum_{j=1}^{Q} a_{j} g\left(\lambda_{j}\right)=g\left(\lambda_{1}\right) \sum_{j=1}^{Q} a_{j}+\int_{\lambda_{1}}^{\lambda_{Q}} g^{\prime}(\lambda) \sum_{j=1}^{Q} a_{j}\left[\lambda_{j} \geqslant \lambda\right] d \lambda .
$$

ДокАЗАТЕльство. Для проверки утверждения леммы достаточно преобразовать интеграл, стоящий в правой части равенства (9.1):

$$
\begin{gathered}
\int_{\lambda_{1}}^{\lambda_{Q}} g^{\prime}(\lambda) \sum_{j=1}^{Q} a_{j}\left[\lambda_{j} \geqslant \lambda\right] d \lambda=\sum_{j=1}^{Q} a_{j} \int_{\lambda_{1}}^{\lambda_{Q}}\left[\lambda \leqslant \lambda_{j}\right] g^{\prime}(\lambda) d \lambda \\
=\sum_{j=1}^{Q} a_{j} \int_{\lambda_{1}}^{\lambda_{j}} g^{\prime}(\lambda) d \lambda=\sum_{j=1}^{Q} a_{j}\left(g\left(\lambda_{j}\right)-g\left(\lambda_{1}\right)\right) .
\end{gathered}
$$

СлеДСТвИЕ 9.2. Предположим, что выполняются условия леммы 9.1 и $a_{1}=\cdots=a_{Q}=1$. Тогда

$$
\sum_{j=1}^{Q} g\left(\lambda_{j}\right)=Q g\left(\lambda_{1}\right)+\int_{\lambda_{1}}^{\lambda_{Q}} g^{\prime}(\lambda) \sum_{j=1}^{Q}\left[\lambda_{j} \geqslant \lambda\right] d \lambda
$$

Лемма 9.3. Пусть $t \geqslant 1$ - действительное число. Тогда количество решеток $\Lambda \subset \mathbb{Z}^{2}$ таких, что $\operatorname{det} \Lambda=q u \lambda_{2}(\Lambda) \geqslant t \sqrt{q}$, можно оценить как $O\left(q^{1+\varepsilon} t^{-2}\right)$.

ДоказАтельство. Так как $\lambda_{2}(\Lambda) \leqslant q$, то утверждение леммы, очевидно, справедливо при $t>\sqrt{q}$. Поэтому будем в дальнейшем предполагать, что $t \leqslant \sqrt{q}$. Пусть $\left(e_{1}, e_{2}\right)$ - приведенный базис решетки $\Lambda$ с определителем $q$ (т. е. $e_{1}-$ кратчайший вектор решетки и проекция $e_{2}$ на $e_{1}$ лежит в пределах от $-e_{1} / 2$ до $\left.e_{1} / 2\right)$. Тогда из неравенства $\left\|e_{2}\right\| \geqslant t \sqrt{q}$ следует, что $\left\|e_{1}\right\| \leqslant 2 \sqrt{q} / t$. Для фиксированного вектора $e_{1}=\left(x_{1}, y_{1}\right)$ конец вектора $e_{2}=\left(x_{2}, y_{2}\right)$ должен лежать на 
прямой $\left\{(x, y): x y_{1}-y x_{1}=q\right\}$ и удовлетворять условию $-\left\|e_{1}\right\|^{2} / 2<\left(e_{1}, e_{2}\right) \leqslant$ $\left\|e_{1}\right\|^{2} / 2$. Поэтому вектор $e_{2}$ можно выбрать $\left(x_{1}, y_{1}\right)$ способами. Значит,

$$
\begin{gathered}
\sum_{\Lambda: \operatorname{det} \Lambda=q}\left[\lambda_{2}(\Lambda) \geqslant t \sqrt{q}\right] \ll \sum_{0 \leqslant x_{1}, y_{1} \leqslant 2 \sqrt{q} / t}\left(x_{1}, y_{1}\right) \\
\ll \sum_{d \mid q, d \leqslant 2 \sqrt{q} / t} d \sum_{0 \leqslant x_{1}, y_{1} \leqslant 2 \sqrt{q} / t}\left[d \mid\left(x_{1}, y_{1}\right)\right] \\
\ll \sum_{d \mid q, d \leqslant 2 \sqrt{q} / t} d\left(\frac{\sqrt{q}}{d t}+1\right)^{2} \ll q^{1+\varepsilon} t^{-2} .
\end{gathered}
$$

Лемма 9.4. Пусть $\beta \leqslant 2$. Тогда

$$
\sum_{\Lambda: \operatorname{det} \Lambda=q} \lambda_{2}^{\beta}(\Lambda) \ll q^{1+\beta / 2+\varepsilon}
$$

ДокАЗАТЕЛьство. Воспользуемся следствием 9.2, полагая $g(\lambda)=\lambda^{\beta}$. В качестве $\lambda_{1}, \ldots, \lambda_{Q}$ выберем числа $\lambda_{2}(\Lambda)$, взятые для всех решеток $\Lambda$ с определителем $q$ и упорядоченные в порядке возрастания. При этом $Q=\sigma(q) \ll q^{1+\varepsilon}$ и $\lambda_{1} \ll \sqrt{q}$ (всегда можно выбрать вектор $e_{1}$ длины порядка $\sqrt{q}$ и дополнить его до приведенного базиса с определителем $q$ ). Значит,

$$
\begin{aligned}
\sum_{\Lambda: \operatorname{det} \Lambda=q} \lambda_{2}^{\beta}(\Lambda) & \ll q^{1+\beta / 2+\varepsilon}+\int_{\sqrt{q}}^{q} \lambda^{\beta-1} \sum_{\Lambda: \operatorname{det} \Lambda=q}\left[\lambda_{2}(\Lambda) \geqslant \lambda\right] d \lambda \\
& \ll q^{1+\beta / 2+\varepsilon}+\sqrt{q} \int_{1}^{\sqrt{q}}(t \sqrt{q})^{\beta-1} \sum_{\Lambda: \operatorname{det} \Lambda=q}\left[\frac{\lambda_{2}(\Lambda)}{\sqrt{q}} \geqslant t\right] d t .
\end{aligned}
$$

Подставляя в последний интеграл оценку из леммы 9.3, приходим к утверждению леммы.

СлЕдСТвиЕ 9.5. Пусть $\beta \leqslant 2 u$

$$
\mathscr{A}_{\ell}(a, q)=\left\{\left(\begin{array}{ll}
a_{1} & a_{2} \\
b_{1} & b_{2}
\end{array}\right):\left(\begin{array}{lll}
a_{1} & a_{2} & a_{3} \\
b_{1} & b_{2} & b_{3} \\
c_{1} & c_{2} & c_{3}
\end{array}\right) \in \mathscr{M}_{\ell}(a, q, P)\right\} .
$$

Тогда

$$
\sum_{a, a_{2}, b_{1}} \lambda_{2}^{\beta}(A)\left[A \in \mathscr{A}_{\ell}(a, q)\right] \ll q^{1+\beta / 2+\varepsilon} .
$$

ДокАзАТЕЛьство. Преобразуем данную сумму:

$$
\begin{aligned}
& \sum_{\Lambda: \operatorname{det} \Lambda=q} \lambda_{2}^{\beta}(\Lambda) \sum_{a, a_{2}, b_{1}}\left[A \in \mathscr{A}_{\ell}(a, q), \Lambda(A)=\Lambda\right] \\
& \leqslant \sum_{\Lambda: \operatorname{det} \Lambda=q} \lambda_{2}^{\beta}(\Lambda) \sum_{a, a_{2}, b_{1}}\left[A \in \mathscr{A}_{\ell}(a, q), \Lambda(A)=\Lambda\right] .
\end{aligned}
$$

Согласно свойству $3^{\circ}$ приведенных матриц, каждому слагаемому во внутренней сумме соответствует некоторый базис Вороного решетки $\Lambda$, причем каждому 
базису Вороного соответствует не более 4 матриц $A$. Следовательно, внутреннюю сумму с точностью до константы можно оценить числом минимальных базисов, то есть

$$
\sum_{a, a_{2}, b_{1}}\left[A \in \mathscr{A}_{\ell}(a, q), \Lambda(A)=\Lambda\right] \ll l\left(\frac{a}{q}\right) \ll \log (q+1) .
$$

Оценивая оставшуюся сумму с помощью леммы 9.4, приходим к утверждению следствия.

СлеДСТвиЕ 9.6. Пусть $\beta \leqslant 2,0<A_{1} \leqslant A_{2}$ u $\alpha$ - произвольное действительное число. Тогда

$$
\sum_{A_{1} \leqslant a<A_{2}} a^{\alpha} \sum_{a_{2}, b_{1}} \lambda_{2}^{\beta}(A)\left[A \in \mathscr{A}_{\ell}(a, q)\right] \ll\left(A_{1}^{\alpha}+A_{2}^{\alpha}\right) q^{1+\beta / 2+\varepsilon} .
$$

ДокАзАТЕльство. Нужная оценка получается из следствия 9.5 с помощью преобразования Абеля

$$
\sum_{A_{1} \leqslant a<A_{2}} f(a) g(a)=f\left(A_{2}\right) \sum_{A_{1} \leqslant a<A_{2}} g(a)-\sum_{A_{1} \leqslant x<A_{2}} \Delta f(x) \sum_{A_{1} \leqslant a \leqslant x} g(a) .
$$

Полагая $f(a)=a^{\alpha}$ и $g(a)=\sum_{a_{2}, b_{1}} \lambda_{2}^{\beta}(A)\left[A \in \mathscr{A}_{\ell}(a, q)\right]$, получаем, что

$$
\begin{aligned}
& \sum_{A_{1} \leqslant a<A_{2}} a^{\alpha} \sum_{a_{2}, b_{1}} \lambda_{2}^{\beta}(A)\left[A \in \mathscr{A}_{\ell}(a, q)\right] \\
& \quad \ll\left(A_{2}^{\alpha}+\sum_{A_{1} \leqslant x<A_{2}} x^{\alpha-1}\right) \sum_{a, a_{2}, b_{1}} \lambda_{2}^{\beta}(A)\left[A \in \mathscr{A}_{\ell}(a, q)\right] \\
& \quad \ll\left(A_{1}^{\alpha}+A_{2}^{\alpha}\right) q^{1+\beta / 2+\varepsilon} .
\end{aligned}
$$

\section{2. Оценка остаточного члена.}

ЛЕмма 9.7. Пусть а - натуральное число, $\alpha, Q_{1}, Q_{2}$ - действительные числа и $0<Q_{1}<Q_{2}$. Тогда

$$
\sum_{Q_{1}<q \leqslant Q_{2}}(a, q) q^{\alpha} \ll\left(Q_{1}^{\alpha+1+\varepsilon}+Q_{2}^{\alpha+1+\varepsilon}\right) a^{\varepsilon} .
$$

ДокАЗАТЕЛЬСтво. Полагая $d=(a, q)$, находим, что

$$
\begin{aligned}
\sum_{Q_{1}<q \leqslant Q_{2}}(a, q) q^{\alpha} & \leqslant \sum_{d \mid a} d \sum_{\substack{Q_{1}<q \leqslant Q_{2} \\
d \mid q}} q^{\alpha} \leqslant \sum_{d \mid a} d^{1+\alpha} \sum_{Q_{1} / d<q_{1} \leqslant Q_{2} / d} q_{1}^{\alpha} \\
& \ll \sum_{d \mid a} d^{1+\alpha}\left(\left(\frac{Q_{1}}{d}\right)^{\alpha+1+\varepsilon}+\left(\frac{Q_{2}}{d}\right)^{\alpha+1+\varepsilon}\right) \\
& \ll\left(Q_{1}^{\alpha+1+\varepsilon}+Q_{2}^{\alpha+1+\varepsilon}\right) a^{\varepsilon} .
\end{aligned}
$$


ПРеДЛОЖЕНИЕ 9.8. Справедливы оценки

$$
\sum_{(a, q) \in \Omega_{i}} R_{i}(a, q, P) \ll P^{2-1 / 34+\varepsilon} \quad(i=1,2,3) .
$$

ДокАЗАтЕЛЬСтво. Просуммируем остаток $R_{1}(a, q, P)=\frac{P^{1+\varepsilon} q}{a^{2}}$ (см. предложение 6.10) по точкам $(a, q) \in \Omega_{1}$ :

$$
\begin{aligned}
\sum_{(a, q) \in \Omega_{1}} \frac{P^{1+\varepsilon} q}{a^{2}} & \ll P^{1+\varepsilon} \sum_{a \leqslant P^{1 / 3}} \frac{1}{a^{2}} \sum_{q \leqslant P^{33 / 68} a^{1 / 2}} q \\
& \ll P^{2-1 / 34+\varepsilon} \sum_{a \leqslant P^{1 / 3}} \frac{1}{a} \ll P^{2-1 / 34+\varepsilon} .
\end{aligned}
$$

Оценим сумму остатков $R_{2}(a, q, P)$. Согласно предложению 7.7

$$
\begin{aligned}
R_{2}(a, q, P) \ll & \frac{(P, q) P^{1+\varepsilon}}{q}\left(\sum_{a_{2}, b_{1}}\left(\frac{r_{1}^{2} \lambda_{2}(A) q^{1 / 2}}{a^{3 / 2}}+\frac{r_{1}^{3} \lambda_{2}(A)}{q^{1 / 2}}\right)+\frac{r_{1} P}{a^{2}}+\frac{P}{r_{1} a}\right) \\
+ & \frac{P^{2}}{q^{2}}\left(r_{2} \frac{q}{a^{2}}+r_{2}^{2} a^{1 / 2}+\frac{q}{r_{2} a}\right)(a, q) a^{\varepsilon} .
\end{aligned}
$$

Выберем значения параметров $r_{1}, r_{2}$ исходя из равенств

$$
\begin{aligned}
r_{1}^{2} \frac{P}{a^{3 / 2} q^{1 / 2} \lambda_{2}(A)} & =\frac{P^{2}}{r_{1} q^{2}}, \\
r_{2}^{2} a^{1 / 2} & =\frac{q}{r_{2} a} \quad\left(\text { при } q \leqslant a^{3}\right), \\
r_{2} \frac{q}{a^{2}} & \left.=\frac{q}{r_{2} a} \quad \text { (при } q>a^{3}\right),
\end{aligned}
$$

предполагая, что $\lambda_{2}(A) \asymp q^{1 / 2}$ :

$$
r_{1}=\left\lfloor P^{1 / 3} a^{1 / 2} q^{-2 / 3}\right\rfloor, \quad r_{2}= \begin{cases}\left\lfloor q^{1 / 3} a^{-1 / 2}\right\rfloor & \text { при } q \leqslant a^{3} \\ \left\lfloor a^{1 / 2}\right\rfloor & \text { при } q>a^{3} .\end{cases}
$$

(Отметим, что в области $\Omega_{2}$ выполняются оценки $r_{1}, r_{2} \leqslant a$, поэтому предложение 7.7 действительно применимо.) Тогда из неравенства $\lambda_{2}(A) \geqslant q^{1 / 2}$ следует, что при $(a, q) \in \Omega_{2}$

$$
\begin{aligned}
R_{2}(a, q, P) \ll( & P, q) P^{5 / 3+\varepsilon} a^{-1 / 2} q^{-11 / 6} \sum_{a_{2}, b_{1}} \lambda_{2}(A) \\
& +(a, q) P^{2+\varepsilon}\left(a^{-1 / 2} q^{-4 / 3}+a^{-3 / 2} q^{-1}\right) .
\end{aligned}
$$


Оценим сумму каждого из слагаемых по парам $(a, q) \in \Omega_{2}$. Для первого слагаемого

$$
\begin{aligned}
P^{5 / 3+\varepsilon} & \sum_{(a, q) \in \Omega_{2}}(P, q) a^{-1 / 2} q^{-11 / 6} \sum_{a_{2}, b_{1}} \lambda_{2}(A) \\
\leqslant & P^{5 / 3+\varepsilon}\left(\sum_{q \leqslant P^{10 / 17}}(P, q) q^{-11 / 6} \sum_{a \geqslant P^{3 / 17}} a^{-1 / 2} \sum_{a_{2}, b_{1}} \lambda_{2}(A)\right. \\
& \left.+\sum_{q>P^{10 / 17}}(P, q) q^{-11 / 6} \sum_{a \gg q^{2} P^{-1}} a^{-1 / 2} \sum_{a_{2}, b_{1}} \lambda_{2}(A)\right) .
\end{aligned}
$$

Применяя к внутренним суммам следствие 9.6 и лемму 9.7, приходим к нужной оценке:

$$
P^{5 / 3+\varepsilon}\left(P^{-3 / 34} \sum_{q \leqslant P^{10 / 17}}(P, q) q^{-1 / 3}+P^{1 / 2} \sum_{q>P^{10 / 17}}(P, q) q^{-4 / 3}\right) \ll P^{2-1 / 34+\varepsilon} .
$$

Просуммируем оставшуюся часть остатка $R_{2}(a, q, P)$. Необходимая оценка также проверяется с помощью леммы 9.7:

$$
\begin{gathered}
\sum_{a \geqslant P^{3 / 17}}(a, q) a^{-3 / 2} \sum_{q \leqslant P} q^{-1} \ll P^{\varepsilon} \sum_{a>P^{3 / 17}} a^{-3 / 2} \ll P^{-3 / 34+\varepsilon}, \\
\sum_{a \geqslant P^{3 / 17}}(a, q) a^{-1 / 2} \sum_{q \geqslant a^{2}} q^{-4 / 3} \ll P^{\varepsilon} \sum_{a \geqslant P^{3 / 17}} a^{-7 / 6} \ll P^{-1 / 34+\varepsilon} .
\end{gathered}
$$

Рассмотрим третий остаток (см. предложение 8.3):

$$
R_{3}(a, q, P) \ll(P, q) P^{\varepsilon}\left(\frac{r D P^{2}}{q^{2}}+\frac{P^{2}}{r a q}+\frac{r^{2} a P}{q} \sum_{a_{2}, b_{1}} \lambda_{2}^{1 / 2}(A)\right) .
$$

Значение $r=\left\lfloor P^{1 / 3} a^{-1 / 3} q^{-5 / 12}\right\rfloor$ выберем исходя из равенства

$$
r^{2} \frac{a P}{q} \lambda_{2}^{1 / 2}(A)=\frac{P^{2}}{r q^{2}}
$$

снова предполагая, что $\lambda_{2}(A) \asymp q^{1 / 2}$. При таком выборе $r$ и при $(a, q) \in \Omega_{3}$

$$
R_{3}(a, q, P) \ll(P, q) P^{5 / 3+\varepsilon} a^{1 / 3} q^{-11 / 6} \sum_{a_{2}, b_{1}} \lambda_{2}^{1 / 2}(A) .
$$

Применяя последовательно следствие 9.6 и лемму 9.7, находим, что

$$
\begin{aligned}
\sum_{(a, q) \in \Omega_{3}} R_{3}(a, q, P) & \ll P^{5 / 3+\varepsilon} \sum_{q \leqslant P^{10 / 17}}(P, q) q^{-11 / 6} \sum_{a \leqslant P^{3 / 17}} a^{1 / 3} \sum_{a_{2}, b_{1}} \lambda_{2}^{1 / 2}(A) \\
& \ll P^{5 / 3+\varepsilon} \sum_{q \leqslant P^{10 / 17}}(P, q) P^{1 / 17} q^{-7 / 12} \ll P^{2-1 / 34+\varepsilon} .
\end{aligned}
$$


9.3. Вспомогательные утверждения. Наряду с функцией Эйлера $\varphi(q)$ будем использовать функции

$$
\varphi_{+}(q)=q \prod_{p \mid q}\left(1+\frac{1}{p}\right) \quad \text { и } \quad \varphi_{2}(q)=\frac{\varphi(q) \varphi_{+}(q)}{q}=q \prod_{p \mid q}\left(1-\frac{1}{p^{2}}\right) .
$$

Лемма 9.9. Пусть $Q \geqslant 2$. Тогда

$$
\begin{aligned}
\sum_{q \leqslant Q} \frac{\varphi_{2}(q)}{q^{2}} & =\frac{1}{\zeta(3)}\left(\log Q+\gamma-\frac{\zeta^{\prime}(3)}{\zeta(3)}\right)+O\left(\frac{1}{Q}\right), \\
\sum_{q \leqslant Q} \frac{\varphi_{2}(q)}{q^{2}} \log q & =\frac{1}{2 \zeta(3)} \log ^{2} Q+c_{0}+O\left(\frac{\log Q}{Q}\right), \\
\sum_{\substack{q \leqslant Q \\
\Delta \mid q}} \frac{\varphi(q)}{q^{2}} & =\frac{\log Q}{\zeta(2) \varphi_{+}(\Delta)}+\psi(\Delta)+O\left(\frac{\log Q}{Q}\right),
\end{aligned}
$$

где $c_{0}-$ абсолютная константа,

$$
\psi(\Delta)=\frac{1}{\Delta} \sum_{d=1}^{\infty} \frac{\mu(d)(d, \Delta)}{d^{2}}\left(\log \frac{(d, \Delta)}{d \Delta}+\gamma\right)
$$

и $\gamma$ - постоянная Эйлера.

ДоказАтельство. Проверим равенство (9.2). Выражая $\varphi_{2}(q)$ через функцию Мёбиуса, приходим к соотношениям

$$
\begin{aligned}
\sum_{q \leqslant Q} \frac{\varphi_{2}(q)}{q^{2}} & =\sum_{q \leqslant Q} \frac{1}{q} \sum_{d \mid q} \frac{\mu(d)}{d^{2}}=\sum_{d \leqslant Q} \frac{\mu(d)}{d^{2}} \sum_{\substack{q \leqslant Q \\
d \mid q}} \frac{1}{q}=\sum_{d \leqslant Q} \frac{\mu(d)}{d^{3}}\left(\log \frac{Q}{d}+\gamma+O\left(\frac{d}{q}\right)\right) \\
& =(\log Q+\gamma)\left(\frac{1}{\zeta(3)}+O\left(\frac{1}{Q^{2}}\right)\right)-\sum_{d \leqslant Q} \frac{\mu(d) \log d}{d^{3}}+O\left(\frac{1}{Q}\right) .
\end{aligned}
$$

Для вычисления возникшей суммы достаточно воспользоваться тем, что

$$
\left(\frac{1}{\zeta(s)}\right)^{\prime}=-\sum_{d=1}^{\infty} \frac{\mu(d) \log d}{d^{s}}=-\frac{\zeta^{\prime}(s)}{\zeta^{2}(s)}
$$

и, в частности,

$$
\sum_{d=1}^{\infty} \frac{\mu(d) \log d}{d^{3}}=\frac{\zeta^{\prime}(3)}{\zeta^{2}(3)}
$$

Преобразуем вторую сумму:

$$
\begin{aligned}
\sum_{q \leqslant Q} \frac{\varphi_{2}(q)}{q^{2}} \log q & =\sum_{q \leqslant Q} \frac{\log q}{q} \sum_{d \mid q} \frac{\mu(d)}{d^{2}}=\sum_{d \leqslant Q} \frac{\mu(d)}{d^{2}} \sum_{\substack{q \leqslant Q \\
d \mid q}} \frac{\log q}{q} \\
& =\sum_{d \leqslant Q} \frac{\mu(d)}{d^{3}} \sum_{q \leqslant Q / d} \frac{\log q+\log d}{q} .
\end{aligned}
$$


Пользуясь равенством (см. [127; раздел 2.21])

$$
\sum_{k \leqslant T} \frac{\log k}{k}=\frac{\log ^{2} T}{2}+\gamma_{1}+O\left(\frac{\log T}{T}\right) \quad(T \geqslant 2)
$$

где $\gamma_{1}$ - первая константа Стилтьеса, находим, что

$$
\sum_{q \leqslant Q} \frac{\varphi_{2}(q)}{q^{2}} \log q=\sum_{d \leqslant Q} \frac{\mu(d)}{d^{3}}\left(\frac{\log ^{2} Q}{2}+\gamma_{1}-\frac{\log ^{2} d}{2}+\gamma \log d\right)+O\left(\frac{\log Q}{Q}\right) .
$$

Полагая

$$
c_{0}=\sum_{d=1}^{\infty} \frac{\mu(d)}{d^{3}}\left(\gamma_{1}-\frac{\log ^{2} d}{2}+\gamma \log d\right)
$$

получаем вторую формулу леммы.

Доказательство равенства (9.4) см. в [24; лемма 9].

Лемма 9.10. Пусть $D$ - натуральное число и $c(q, D)$ определено равенством (6.18). Тогда для суммы

$$
S_{D}\left(Q_{1}, Q_{2}\right)=\sum_{\substack{Q_{1} \leqslant q \leqslant Q_{2} \\ D \mid q}} \frac{\varphi(q)}{q^{2}} c(q, D)
$$

справедлива асимптотическая формула

$$
S_{D}\left(Q_{1}, Q_{2}\right)=\frac{\log \left(Q_{2} / Q_{1}\right)}{\zeta(2)}+O\left(D^{1+\varepsilon} Q_{1}^{-1+\varepsilon}\right) .
$$

ДокАЗАТЕЛЬство. Полагая $q=D q_{1}$, для данной суммы получаем представление

$$
S_{D}\left(Q_{1}, Q_{2}\right)=\frac{1}{\varphi(D)} \sum_{Q_{1} \leqslant D q_{1} \leqslant Q_{2}} \frac{\varphi\left(D q_{1}\right) \varphi\left(\left(D, q_{1}\right)\right)}{\left(D, q_{1}\right) q_{1}^{2}}
$$

Преобразуем полученную сумму, вводя параметры $\Delta=\left(D, q_{1}\right), q_{2}=q_{1} \Delta^{-1}$ и $q_{3}=q_{2} \delta^{-1}$ :

$$
\begin{aligned}
S_{D}\left(Q_{1}, Q_{2}\right) & =\frac{1}{\varphi(D)} \sum_{\Delta \mid D} \frac{\varphi(\Delta)}{\Delta^{3}} \sum_{\substack{Q_{1} \leqslant D \Delta q_{2} \leqslant Q_{2} \\
\left(D / \Delta, q_{2}\right)=1}} \frac{\varphi\left(D \Delta q_{2}\right)}{q_{2}^{2}} \\
& =\frac{1}{\varphi(D)} \sum_{\Delta \mid D} \frac{\varphi(\Delta)}{\Delta^{3}} \sum_{\substack{Q_{1} \leqslant D \Delta q_{2} \leqslant Q_{2} \\
q_{2}^{2}}} \frac{\varphi\left(D \Delta q_{2}\right)}{\delta \mid\left(D / \Delta, q_{2}\right)} \mu(\delta) \\
& =\frac{1}{\varphi(D)} \sum_{\Delta \mid D} \frac{\varphi(\Delta)}{\Delta^{3}} \sum_{\delta \mid D / \Delta} \frac{\mu(\delta)}{\delta^{2}} \sum_{Q_{1} \leqslant D \Delta \delta q_{3} \leqslant Q_{2}} \frac{\varphi\left(D \Delta \delta q_{3}\right)}{q_{3}^{2}} \\
& =\frac{D^{2}}{\varphi(D)} \sum_{\delta \Delta \mid D} \frac{\varphi(\Delta) \mu(\delta)}{\Delta} \sum_{\substack{Q_{1} \leqslant q \leqslant Q_{2} \\
D \Delta \delta \mid q}} \frac{\varphi(q)}{q^{2}}
\end{aligned}
$$


Применим к внутренней сумме равенство (9.4):

$$
\begin{aligned}
S_{D}\left(Q_{1}, Q_{2}\right) & =\frac{D^{2}}{\varphi(D)} \sum_{\delta \Delta \mid D} \frac{\varphi(\Delta) \mu(\delta)}{\Delta}\left(\frac{\log \left(Q_{2} / Q_{1}\right)}{\zeta(2) \varphi_{+}(D \Delta \delta)}+O\left(Q_{1}^{-1+\varepsilon}\right)\right) \\
& =\frac{\log Q_{2} / Q_{1}}{\zeta(2)} \frac{D^{2}}{\varphi(D)} \sum_{\delta \Delta \mid D} \frac{\varphi(\Delta)}{\Delta} \frac{\mu(\delta)}{\Delta \delta \varphi_{+}(D)}+O\left(D^{1+\varepsilon} Q_{1}^{-1+\varepsilon}\right) \\
& =\frac{\log Q_{2} / Q_{1}}{\zeta(2)} \frac{D}{\varphi_{2}(D)} \sum_{\delta \Delta \mid D} \frac{\varphi(\Delta)}{\Delta^{2}} \frac{\mu(\delta)}{\delta}+O\left(D^{1+\varepsilon} Q_{1}^{-1+\varepsilon}\right) \\
& =\frac{\log Q_{2} / Q_{1}}{\zeta(2)} \frac{D}{\varphi_{2}(D)} \sum_{\Delta \mid D} \frac{\varphi(\Delta)}{\Delta^{2}} \frac{\varphi(D / \Delta)}{D / \Delta}+O\left(D^{1+\varepsilon} Q_{1}^{-1+\varepsilon}\right) .
\end{aligned}
$$

Подставляя в последнюю формулу равенство (см. [24; лемма 8])

$$
\sum_{\Delta \mid D} \frac{\varphi(\Delta) \varphi(D / \Delta)}{\Delta}=\varphi_{2}(D)
$$

приходим к утверждению леммы.

9.4. Подсчет главного члена. Для завершения доказательства теоремы 5.5 остается перейти к интегрированию по переменным из первой строки матрицы $X$. Предварительно необходимо выполнить суммирование по переменной $q$.

Условия $a \leqslant b \leqslant c$, которым удовлетворяют матрицы $X \in \mathscr{M}_{\ell}(a, q, P)$, не инвариантны относительно левого действия $D_{3}(\mathbb{R})$. Выразим $c$ и $b$ из равенств

$$
a b c=\frac{P}{\operatorname{det} X^{\prime \prime \prime}}, \quad a b=\frac{q}{\operatorname{det} A^{\prime \prime}},
$$

где $A^{\prime \prime}=\left(\begin{array}{cc}1 & \alpha_{2} \\ \beta_{1} & 1\end{array}\right)$. Тогда неравенства $a \leqslant b \leqslant c$ после перехода к множеству $\mathscr{M}_{\ell}^{\prime \prime \prime}(a, q, P)$ примут вид

$$
a \leqslant \frac{q}{a \operatorname{det} A^{\prime \prime}} \leqslant \frac{P \operatorname{det} A^{\prime \prime}}{q \operatorname{det} X^{\prime \prime \prime}} .
$$

Поэтому при заданных коэффициентах матрицы $X^{\prime \prime \prime}$ для каждого $i=1,2,3$ область $\Omega_{i}\left(X^{\prime \prime \prime}\right)$, в которой могут меняться $a$ и $q$, задается равенством

$$
\Omega_{i}\left(X^{\prime \prime \prime}\right)=\left\{(a, q) \in \Omega_{i}: a^{2} \operatorname{det} A^{\prime \prime} \leqslant q \leqslant\left(\frac{a P}{\operatorname{det} X^{\prime \prime \prime}}\right)^{1 / 2} \operatorname{det} A^{\prime \prime}\right\} \quad(i=1,2,3) .
$$

ЗАмечАниЕ 9.11. Как и в двумерном случае (см. п. 4.7), для тех пар $(a, q)$, для которых множества $\mathscr{M}_{\ell}(a, q, P)$ пусты, значения функций $\rho_{2,3}(a, q)$ естественно доопределить нулем. Поэтому будем считать, что значения $\rho_{2,3}(a, q)$ определены для всех натуральных $a$ и $q$. 
ПреДЛОЖЕНИЕ 9.12. Для величины $\mathscr{N}_{\ell}^{(2)}(P)$, определенной равенством (5.9), справедлива асимптотическая формула

$$
\begin{aligned}
\frac{\mathscr{N}_{\ell}^{(2)}(P)}{\varphi^{2}(P)}= & \frac{1}{\zeta(2)} \int_{\mathbb{R}^{6}} \frac{d \boldsymbol{\alpha} d \boldsymbol{\beta} d \boldsymbol{\gamma}\left[X^{\prime \prime \prime} \in \mathscr{M}_{\ell}^{\prime \prime \prime}\right]}{\left(\operatorname{det} X^{\prime \prime \prime}\right)^{3}} \\
& \quad \times \sum_{a}\left(\frac{\varphi_{2}(a)}{a^{2}} \int_{\mathbb{R}} \frac{d q}{q}\left[(a, q) \in \Omega_{2}\left(X^{\prime \prime \prime}\right)\right]+\rho_{2}(a)\right)+O\left(P^{-1 / 34+\varepsilon}\right),
\end{aligned}
$$

где $\rho_{2}(a) \ll a^{-2+\varepsilon}$.

ДокАзАТЕЛьСтво. Согласно предложениям 7.7 и 9.8

$$
N_{\ell}^{(2)}(P)=\varphi^{2}(P) \int_{\mathbb{R}^{6}} \frac{d \boldsymbol{\alpha} d \boldsymbol{\beta} d \boldsymbol{\gamma}}{\left(\operatorname{det} X^{\prime \prime \prime}\right)^{3}} S_{\ell}^{(2)}(P, \boldsymbol{\alpha}, \boldsymbol{\beta}, \boldsymbol{\gamma})+O\left(P^{-1 / 34+\varepsilon}\right)
$$

где

$$
S_{\ell}^{(2)}(P, \boldsymbol{\alpha}, \boldsymbol{\beta}, \boldsymbol{\gamma})=\sum_{(a, q) \in \Omega_{2}} \frac{\varphi(q)}{q^{2}} \frac{K_{a}^{\times}(q)}{a^{2}}\left[X^{\prime \prime \prime} \in \mathscr{M}_{\ell}^{\prime \prime \prime}(a, q, P)\right]
$$

Множество $\widetilde{\mathscr{M}^{\prime \prime \prime}}$ получается из $\mathscr{M}_{\ell}^{\prime \prime \prime}(a, q, P)$ отбрасыванием условий $(9.7)$ с сохранением остальных (инвариантных) неравенств. Поэтому

$$
\left[X^{\prime \prime \prime} \in \mathscr{M}_{\ell}^{\prime \prime \prime}(a, q, P),(a, q) \in \Omega_{i}\right]=\left[X^{\prime \prime \prime} \in \mathscr{M}_{\ell}^{\prime \prime \prime},(a, q) \in \Omega_{i}\left(X^{\prime \prime \prime}\right)\right]
$$

и

$$
S_{\ell}^{(2)}(P, \boldsymbol{\alpha}, \boldsymbol{\beta}, \boldsymbol{\gamma})=\left[X^{\prime \prime \prime} \in \mathscr{M}_{\ell}^{\prime \prime \prime}\right] \sum_{a, q} \frac{\varphi(q)}{q^{2}} \frac{K_{a}^{\times}(q)}{a^{2}}\left[(a, q) \in \Omega_{2}\left(X^{\prime \prime \prime}\right)\right]
$$

Применим к возникшей сумме равенство (см. [24; следствие 3])

$$
\sum_{Q_{1} \leqslant q \leqslant Q_{2}} \frac{\varphi(q)}{q^{2}} \frac{K_{a}^{\times}(q)}{a^{2}}=\frac{1}{\zeta(2)} \frac{\varphi_{2}(a)}{a^{2}} \int_{Q_{1}}^{Q_{2}} \frac{d q}{q}+O\left(a^{-1+\varepsilon} Q_{1}^{-1+\varepsilon}\right) .
$$

Для нижнего предела суммирования $Q_{1}$ выполняется оценка $Q_{1} \gg a^{2}$. Поэтому

$$
S_{\ell}^{(2)}(P, \boldsymbol{\alpha}, \boldsymbol{\beta}, \boldsymbol{\gamma})=\frac{\left[X^{\prime \prime \prime} \in \mathscr{M}_{\ell}^{\prime \prime \prime}\right]}{\zeta(2)} \sum_{a}\left(\frac{\varphi_{2}(a)}{a^{2}} \int_{\mathbb{R}} \frac{d q}{q}\left[(a, q) \in \Omega_{2}\left(X^{\prime \prime \prime}\right)\right]+O\left(a^{-3+\varepsilon}\right)\right)
$$

Подставляя последнюю формулу в (9.8), приходим к нужному равенству. 
Лемма 9.13. Пусть $\Omega \subset[-a, a]^{2}$ и граница области $\Omega$ имеет длину $O(a)$. Тогда для суммы

$$
S(\Omega)=\sum_{\substack{\left(a_{2}, a_{3}\right) \in \Omega \\\left(a, a_{2}, a_{3}\right)=1}} \frac{1}{\left(\operatorname{det} X^{\prime \prime}\right)^{3}}
$$

справедлива асимптотическая формула

$$
S(\Omega)=\frac{\varphi_{2}(a)}{a^{2}} \int_{\mathbb{R}^{2}} \frac{\left[\left(\alpha_{2}, \alpha_{3}\right) \in a^{-1} \Omega\right] d \boldsymbol{\alpha}}{\left(\operatorname{det} X^{\prime \prime \prime}\right)^{3}}+O\left(a^{-2+\varepsilon}\right) .
$$

ДоКАЗАТЕЛЬСТво. Избавимся от условия взаимной простоты с помощью функции Мёбиуса:

$$
S(\Omega)=\sum_{d \mid a} \mu(d) \sum_{\substack{\left(a_{2}, a_{3}\right) \in \Omega \\
d\left|a_{2}, d\right| a_{3}}}\left|\begin{array}{ccc}
a & a_{2} & a_{3} \\
\beta_{1} & \beta_{2} & \beta_{3} \\
\gamma_{1} & \gamma_{2} & \gamma_{3}
\end{array}\right|^{-3}=\sum_{d \mid a} \frac{\mu(d)}{d^{3}} \sum_{\left(a_{2}^{\prime}, a_{3}^{\prime}\right) \in d^{-1} \Omega}\left|\begin{array}{ccc}
a^{\prime} & a_{2}^{\prime} & a_{3}^{\prime} \\
\beta_{1} & \beta_{2} & \beta_{3} \\
\gamma_{1} & \gamma_{2} & \gamma_{3}
\end{array}\right|^{-3} .
$$

Пусть

$$
G(u, v)=\left|\begin{array}{ccc}
a^{\prime} & u & v \\
\beta_{1} & \beta_{2} & \beta_{3} \\
\gamma_{1} & \gamma_{2} & \gamma_{3}
\end{array}\right|^{-3}
$$

Тогда

$$
G(u, v) \asymp\left(a^{\prime}\right)^{-3}, \quad \frac{\partial G}{\partial u} \ll \frac{1}{\left(a^{\prime}\right)^{4}}, \quad \frac{\partial G}{\partial v} \ll \frac{1}{\left(a^{\prime}\right)^{4}} .
$$

Следовательно,

$$
G(u, v)=\int_{[0,1]^{2}} G\left(u+t_{1}, v+t_{2}\right) d t_{1} d t_{2}+O\left(\frac{1}{\left(a^{\prime}\right)^{4}}\right) .
$$

Число единичных квадратов, пересекаемых границей области $d^{-1} \Omega$, есть $O\left(a^{\prime}\right)$. В каждом из таких квадратов можно воспользоваться формулой с тривиальной оценкой остаточного члена

$$
G(u, v)=\int_{[0,1]^{2}} G\left(u+t_{1}, v+t_{2}\right)\left[\left(u+t_{1}, v+t_{2}\right) \in d^{-1} \Omega\right] d t_{1} d t_{2}+O\left(\frac{1}{\left(a^{\prime}\right)^{3}}\right) .
$$

Значит,

$$
\begin{aligned}
S(\Omega) & =\sum_{d \mid a} \frac{\mu(d)}{d^{3}}\left(\int_{d^{-1} \Omega}\left|\begin{array}{ccc}
a^{\prime} & a_{2}^{\prime} & a_{3}^{\prime} \\
\beta_{1} & \beta_{2} & \beta_{3} \\
\gamma_{1} & \gamma_{2} & \gamma_{3}
\end{array}\right|^{-3} d a_{2}^{\prime} d a_{3}^{\prime}+O\left(d^{2} a^{-2}\right)\right) \\
& =\frac{1}{a} \sum_{d \mid a} \frac{\mu(d)}{d^{2}} \int_{a^{-1} \Omega}\left|\begin{array}{ccc}
1 & \alpha_{2} & \alpha_{3} \\
\beta_{1} & \beta_{2} & \beta_{3} \\
\gamma_{1} & \gamma_{2} & \gamma_{3}
\end{array}\right|^{-3} d \boldsymbol{\alpha}+O\left(a^{-2+\varepsilon}\right) \\
& =\frac{\varphi_{2}(a)}{a^{2}} \int_{a^{-1} \Omega}\left|\begin{array}{ccc}
1 & \alpha_{2} & \alpha_{3} \\
\beta_{1} & \beta_{2} & \beta_{3} \\
\gamma_{1} & \gamma_{2} & \gamma_{3}
\end{array}\right|^{-3} d \boldsymbol{\alpha}+O\left(a^{-2+\varepsilon}\right) .
\end{aligned}
$$


ПРеДЛОЖениЕ 9.14. Справедливы равенства

$$
\begin{aligned}
\frac{\mathscr{N}_{\ell}^{(1,3)}(P)}{\varphi^{2}(P)}= & \frac{1}{\zeta(2)} \int_{\mathbb{R}^{6}} \frac{d \boldsymbol{\alpha} d \boldsymbol{\beta} d \boldsymbol{\gamma}\left[X^{\prime \prime \prime} \in \mathscr{M}_{\ell}^{\prime \prime \prime}\right]}{\left(\operatorname{det} X^{\prime \prime \prime}\right)^{3}} \\
& \times \sum_{a} \frac{\varphi_{2}(a)}{a^{2}}\left(\int_{\mathbb{R}} \frac{d q}{q}\left[(a, q) \in \Omega_{1,3}\left(X^{\prime \prime \prime}\right)\right]+\rho_{1,3}(a)\right) \\
& +O\left(P^{-1 / 34+\varepsilon}\right)
\end{aligned}
$$

где $\rho_{1,3}(a) \ll a^{-5 / 4+\varepsilon}$.

ДоКАЗАТЕЛЬСтво. Согласно предложениям 6.10, 8.3 и 9.8

$$
\mathscr{N}_{\ell}^{(1,3)}(P)=\varphi^{2}(P)\left(\int_{\mathbb{R}^{4}} d \boldsymbol{\beta} d \boldsymbol{\gamma} S_{\ell}^{(1,3)}(P, \boldsymbol{\beta}, \boldsymbol{\gamma})+\sum_{(a, q) \in \Omega_{1,3}} \rho_{1,3}(a, q)\right)+O\left(P^{-1 / 34+\varepsilon}\right)
$$

где

$$
S_{\ell}^{(1,3)}(P, \boldsymbol{\beta}, \boldsymbol{\gamma})=\sum_{(a, q) \in \Omega_{1,3}} \sum_{a_{2}, a_{3}}^{\#} \frac{\varphi(q)}{q^{2}} c(q, D) \frac{\left[X^{\prime \prime} \in \mathscr{M}_{\ell}^{\prime \prime}(a, q, P)\right]}{\left(\operatorname{det} X^{\prime \prime}\right)^{3}}
$$

Из равенства (9.9) следует, что

$$
S_{\ell}^{(1,3)}(P, \boldsymbol{\beta}, \boldsymbol{\gamma})=\sum_{a, a_{2}, a_{3}}^{\#} \frac{\left[X^{\prime \prime \prime} \in \mathscr{M}_{\ell}^{\prime \prime \prime}\right]}{\left(a \operatorname{det} X^{\prime \prime \prime}\right)^{3}} \sum_{q} \frac{\varphi(q)}{q^{2}} c(q, D)\left[(a, q) \in \Omega_{1,3}\left(X^{\prime \prime \prime}\right)\right]
$$

K сумме по $q$ применим лемму 9.10 :

$$
\begin{aligned}
S_{\ell}^{(1,3)}(P, \boldsymbol{\beta}, \boldsymbol{\gamma})= & \frac{1}{\zeta(2)} \sum_{a, a_{2}, a_{3}}^{\#} \frac{\left[X^{\prime \prime \prime} \in \mathscr{M}_{\ell}^{\prime \prime \prime}\right]}{\left(a \operatorname{det} X^{\prime \prime \prime}\right)^{3}} \\
& \times\left(\int_{\mathbb{R}} \frac{d q}{q}\left[(a, q) \in \Omega_{1,3}\left(X^{\prime \prime \prime}\right)\right]+O\left(D a^{-2+\varepsilon}\right)\right) .
\end{aligned}
$$

По лемме 9.7

$$
\sum_{a_{2}, a_{3} \leqslant a} D a^{-5+\varepsilon}=\sum_{a_{2}, a_{3} \leqslant a}\left(a_{2}, a\right) a^{-5+\varepsilon} \ll a^{-3+\varepsilon}
$$

Значит,

$$
\begin{aligned}
S_{\ell}^{(1,3)}(P, \boldsymbol{\beta}, \boldsymbol{\gamma})= & \frac{1}{\zeta(2)} \sum_{a}\left(\frac{1}{a^{3}} \int_{\mathbb{R}} \frac{d q}{q} \sum_{a_{2}, a_{3}}^{\#} \frac{\left[X^{\prime \prime \prime} \in \mathscr{M}_{\ell}^{\prime \prime \prime}\right]}{\left(\operatorname{det} X^{\prime \prime \prime}\right)^{3}}\right. \\
& \left.\times\left[(a, q) \in \Omega_{1,3}\left(X^{\prime \prime \prime}\right)\right]+O\left(a^{-3+\varepsilon}\right)\right) .
\end{aligned}
$$


Применяя лемму 9.13, приходим к асимптотической формуле

$$
\begin{aligned}
S_{\ell}^{(1,3)}(P, \boldsymbol{\beta}, \boldsymbol{\gamma})= & \frac{1}{\zeta(2)} \sum_{a}\left(\frac{\varphi_{2}(a)}{a^{2}}\right. \\
& \left.\times \int_{\mathbb{R}^{2}} d \boldsymbol{\alpha} \frac{\left[X^{\prime \prime \prime} \in \mathscr{M}_{\ell}^{\prime \prime \prime}\right]}{\left(\operatorname{det} X^{\prime \prime \prime}\right)^{3}} \int_{\mathbb{R}} \frac{d q}{q}\left[(a, q) \in \Omega_{1,3}\left(X^{\prime \prime \prime}\right)\right]+O\left(a^{-2+\varepsilon}\right)\right) .
\end{aligned}
$$

Кроме того, по лемме 9.7

$$
\begin{aligned}
& \sum_{q} \rho_{1}(a, q)\left[(a, q) \in \Omega_{1}\right] \ll \sum_{q \geqslant a^{2}}(a, q) a^{-1 / 4} q^{-3 / 2+\varepsilon} \ll a^{-5 / 4+\varepsilon}, \\
& \sum_{q} \rho_{3}(a, q)\left[(a, q) \in \Omega_{3}\right] \ll \sum_{q \geqslant a^{4}} a q^{-2+\varepsilon} \ll a^{-3+\varepsilon} .
\end{aligned}
$$

Подставляя (9.11), (9.12) и (9.13) в формулу (9.10), приходим к нужному равенству.

СлеДСТвИЕ 9.15. Справедлива асимптотическая формула

$$
\begin{aligned}
\frac{\mathscr{N}_{\ell}(P)}{\varphi^{2}(P)}= & \frac{1}{\zeta(2)} \int_{\mathbb{R}^{6}} \frac{d \boldsymbol{\alpha} d \boldsymbol{\beta} d \boldsymbol{\gamma}\left[X^{\prime \prime \prime} \in \mathscr{M}_{\ell}^{\prime \prime \prime}\right]}{\left(\operatorname{det} X^{\prime \prime \prime}\right)^{3}} \\
& \times \sum_{a} \frac{\varphi_{2}(a)}{a^{2}}\left(\int_{\mathbb{R}} \frac{d q}{q}\left[(a, q) \in \Omega\left(X^{\prime \prime \prime}\right)\right]+\rho(a)\right)+O\left(P^{-1 / 34+\varepsilon}\right),
\end{aligned}
$$

где $\rho(a) \ll a^{-5 / 4+\varepsilon}$.

Для доказательства достаточно подставить асимптотические формулы из предложений 9.12 и 9.14 в равенство (5.8).

ДокАЗАТЕЛЬСТво теоремы 5.5. Так как

$$
\sum_{a \ll P^{1 / 3}} \rho(a)=\sum_{a=1}^{\infty} \rho(a)+O\left(P^{-1 / 12+\varepsilon}\right),
$$

то, согласно следствию 9.15,

$$
\begin{gathered}
\frac{\mathscr{N}_{\ell}(P)}{\varphi^{2}(P)}=\frac{1}{\zeta(2)} \int_{\mathbb{R}^{6}} \frac{d \boldsymbol{\alpha} d \boldsymbol{\beta} d \boldsymbol{\gamma}\left[X^{\prime \prime \prime} \in \mathscr{M}_{\ell}^{\prime \prime \prime}\right]}{\left(\operatorname{det} X^{\prime \prime \prime}\right)^{3}} \sum_{a} \frac{\varphi_{2}(a)}{a^{2}} \int_{\mathbb{R}} \frac{d q}{q}\left[(a, q) \in \Omega\left(X^{\prime \prime \prime}\right)\right] \\
+\sum_{a=1}^{\infty} \rho(a)+O\left(P^{-1 / 34+\varepsilon}\right) .
\end{gathered}
$$

Преобразуем интеграл по переменной $q$ :

$$
\begin{array}{r}
\int_{\mathbb{R}} \frac{d q}{q}\left[(a, q) \in \Omega\left(X^{\prime \prime \prime}\right)\right]=\int_{\mathbb{R}} \frac{d q}{q}\left[a^{2} \operatorname{det} A^{\prime \prime} \leqslant q \leqslant\left(\frac{a P}{\operatorname{det} X^{\prime \prime \prime}}\right)^{1 / 2} \operatorname{det} A^{\prime \prime}\right] \\
=\int_{\mathbb{R}} \frac{d q}{q}\left[a^{2} \leqslant q \leqslant\left(\frac{a P}{\operatorname{det} X^{\prime \prime \prime}}\right)^{1 / 2}\right]=\frac{1}{2} \log P-\frac{3}{2} \log a-\frac{1}{2} \log X^{\prime \prime \prime} .
\end{array}
$$

Подставляя полученное выражение в формулу для $\mathscr{N}_{\ell}(P)$ и пользуясь равенствами (9.2), (9.3), приходим к утверждению теоремы. 


\section{Список литературы}

[1] W.M. Schmidt, "The density of integer points on homogeneous varieties", Acta Math., 154:3-4 (1985), 243-296.

[2] B. Z. Moroz, "On the number of integral points on a norm-form variety in a cube-like domain", J. Number Theory, 27:1 (1987), 106-110.

[3] J. Franke, Yu. I. Manin, Yu. Tschinkel, "Rational points of bounded height on Fano varieties", Invent. Math., 95:2 (1989), 421-435.

[4] Yu. V. Linnik, "Additive problems and eigenvalues of the modular operators", Proceedings of the International Congress of Mathematicians (Stockholm, 1962), Inst. Mittag-Leffler, Djursholm, 1963, 270-284.

[5] P. C. Sarnak, "Diophantine problems and linear groups", Proceedings of the International Congress of Mathematicians, v. I (Kyoto, 1990), Math. Soc. Japan, Tokyo, 1991, 459-471.

[6] W. Duke, Z. Rudnick, P. Sarnak, "Density of integer points on affine homogeneous varieties", Duke Math. J., 71:1 (1993), 143-179.

[7] В. А. Быковский, "О распределении целых точек на детерминантной поверхности”, Далъневост. матем. журн., 14:2 (2014), 156-159.

[8] Ю.В. Линник, Б.Ф. Скубенко, "Асимптотическое распределение целочисленных матриц третьего порядка", Вестн. Ленингр. ун-та. Сер. матем., мех., астрон., 3:13 (1964), 25-36.

[9] Ю. В. Линник, Эргодические свойства алгебрачческих полей, Изд-во Ленингр. ун-та, Л., 1967, 208 с.; англ. пер.: Yu. V. Linnik, Ergodic properties of algebraic fields, Ergeb. Math. Grenzgeb., 45, Springer-Verlag, New York, 1968, ix+192 pp.

[10] Wee Teck Gan, Hee Oh, "Equidistribution of integer points on a family of homogeneous varieties: a problem of Linnik", Compositio Math., 136:3 (2003), 323-352.

[11] Б. Ф. Скубенко, "К распределению целочисленных матриц и вычислению объема фундаментальной области унимодулярной группы матриц", Алгебраическал теория чисел и представления, Сборник работ, Тр. МИАН СССР, 80, Наука, М.-Л., 1965, 129-144; англ. пер.: В.F. Skubenko, "The distribution of integer matrices and calculation of the volume of the fundamental domain of a unimodular group of matrices", Proc. Steklov Inst. Math., 80 (1965), 147-163.

[12] А. М. Истамов, "Асимптотика целочисленных матриц второго порядка, лежащих в данной гиперболической области и принадлежащих заданному классу вычетов", Исследования по теории чисел. 6, Зап. науч. сем. ЛОМИ, 93, Наука, Ленингр. отд., Л., 1980, 25-29; англ. пер.: А. M. Istamov, "Asymptotics of second-order integral matrices lying in a given hyperbolic region and belonging to a given residue class", J. Soviet Math., 19:2 (1982), 1085-1088.

[13] А. М. Истамов, "Асимптотическое распределение целочисленных матриц $n$-го порядка, принадлежащих заданному классу вычетов", Аналитическая теория чисел и теория функиий. 4, Зап. науч. сем. ЛОМИ, 112, Наука, Ленингр. отд., Л., 1981, 85-111; англ. пер.: А. М. Istamov, "Asymptotic distribution of $n$-th-order integral matrices belonging to a given residue class", J. Soviet Math., 25:2 (1984), 1030-1051.

[14] Е. В. Подсыпанин, "Распределение целых точек на детерминантной поверхности", Исследования по теории чисел. 6, Зап. науч. сем. ЛОМИ, 93, Наука, Ленингр. отд., Л., 1980, 30-40; англ. пер.: Е. V. Podsypanin, "Distribution of integral points on the determinant surface", J. Soviet Math., 19:2 (1982), 1088-1095.

[15] Н. М. Коробов, Тригонометрические суммъ и их приложения, Наука, М., 1989, 240 с.; англ. пер.: N. M. Korobov, Exponential sums and their applications, Math. Appl. (Soviet Ser.), 80, Kluwer Acad. Publ., Dordrecht, 1992, xvi+209 pp. 
[16] I. E. Shparlinski, "Modular hyperbolas", Jpn. J. Math., 7:2 (2012), 235-294.

[17] Г. Вороной, Об одном обобщении алгорифма непрерывных дробей, Типография Варшавского Учебного Округа, Варшава, 1896, 221 с.

[18] Г. Ф. Вороной, Собрание сочинений, т. 1, Изд-во АН УССР, Киев, 1952, 399 с.

[19] H. Minkowski, "Généralisation de la théorie des fractions continues", Ann. Sci. École Norm. Sup. (3), 13 (1896), 41-60.

[20] Б. Н. Делоне, Д. К. Фаддеев, “Теория иррациональностей третьей степени”, Тр. Матем. ин-та им. В. А. Стеклова, 11, Изд-во АН СССР, М.-Л., 1940, 3-340.

[21] Б. Н. Делоне, Петербургская школа теории чисел, Изд-во АН СССР, М.-Л., 1947, $421 \mathrm{c}$.

[22] H. Hancock, Development of the Minkowski geometry of numbers, v. 1, Dover Publications, Inc., New York, 1964, xix+452 pp.

[23] А. В. Устинов, "О распределении решений детерминантного уравнения", Матем. сб., 206:7 (2015), 103-134.

[24] В. И. Берник, А. В. Устинов, "О распределении точек модулярной гиперболы”, Дальневост. матем. журн., 14:2 (2014), 141-155.

[25] H. Heilbronn, "On the average length of a class of finite continued fractions", $A b$ handlungen aus Zahlentheorie und Analysis, Deutscher Verlag der Wissenschaften, Berlin, 1968, 87-96.

[26] J. W. Porter, "On a theorem of Heilbronn", Mathematika, 22:1 (1975), 20-28.

[27] А. А. Илларионов, "Среднее количество относительных минимумов трехмерных целочисленных решеток фиксированного определителя", Изв. РАН. Сер. матем., 76:3 (2012), 111-138; англ. пер.: А. A. Illarionov, "The average number of relative minima of three-dimensional integer lattices of a given determinant", $I z v$. Math., 76:3 (2012), 535-562.

[28] А.А. Илларионов, "О статистических свойствах многогранников Клейна трехмерных целочисленных решеток”, Матем. сб., 204:6 (2013), 23-46; англ. пер.: А.A. Illarionov, "On the statistical properties of Klein polyhedra in three-dimensional lattices", Sb. Math., 204:6 (2013), 801-823.

[29] А.А. Илларионов, "Многомерное обобщение теоремы Хейльбронна о средней длине конечной непрерывной дроби”, Матем. сб., 205:3 (2014), 119-132; англ. пер.: A. A. Illarionov, "A multidimensional generalization of Heilbronn's theorem on the average length of a finite continued fraction", Sb. Math., 205:3 (2014), 419-431.

[30] А.А. Илларионов, "О среднем количестве наилучших приближений линейных форм”, Изв. РАН. Сер. матем., 78:2 (2014), 61-86; англ. пер.: A. A. Illarionov, "On the average number of best approximations of linear forms", Izv. Math., 78:2 (2014), 268-292.

[31] Б. В. Гнеденко, Курс теории вероятностей, 6-е изд., перераб. и доп., Наука, M., 1988, 448 с.; англ. пер.: В. V. Gnedenko, The theory of probability, Moscow, Mir, 1988, $392 \mathrm{pp}$.

[32] R. O. Kuz'min, "Sur un problème de Gauss", Atti del Congresso Internazionale dei Matematici, v. 6 (Bologna, 1928), Zanichelli, Bologna, 1932, 83-89.

[33] P. Lévy, "Sur les lois de probabilité dont dépendent les quotients complets et incomplets d'une fraction continue", Bull. Soc. Math. France, 57 (1929), 178-194.

[34] E. Wirsing, "On the theorem of Gauss-Kusmin-Lévy and a Frobenius-type theorem for function spaces", Acta Arith., 24 (1973/1974), 507-528.

[35] К.И. Бабенко, "Об одной задаче Гаусса", Докл. АН ССCP, 238:5 (1978), 1021-1024; англ. пер.: K. I. Babenko, "On a problem of Gauss", Soviet Math. Dokl., 19:1 (1978), 136-140. 
[36] M. Iosifescu, C. Kraaikamp, Metrical theory of continued fractions, Math. Appl., 547, Kluwer Acad. Publ., Dordrecht, 2002, xx+383 pp.

[37] G. Lochs, "Statistik der Teilnenner der zu den echten Brüchen gehörigen regelmäßigen Kettenbrüche", Monatsh. Math., 65:1 (1961), 27-52.

[38] R. Adler, M. Keane, M. Smorodinsky, "A construction of a normal number for the continued fraction transformation", J. Number Theory, 13:1 (1981), 95-105.

[39] В.И. Арнольд, Задачи Арнольда, Фазис, М., 2000, х +452 с.; англ. пер.: V.I. Arnold, Arnold's problems, Springer-Verlag, Berlin-Heidelberg; PHASIS, Moscow, 2004, xvi+639 pp.

[40] М.О. Авдеева, В.А. Быковский, Решение задачи Арнолъда о статистиках Гаусса-Кузъмина, Препринт ИПМ № 2002-8, Изд-во “Дальнаука" ДВО РАН, Владивосток, 2002, 12 с.

[41] М. О. Авдеева, "О статистиках неполных частных конечных цепных дробей", Функи. анализ и его прил., 38:2 (2004), 1-11; англ. пер.: М. O. Avdeeva, "On the statistics of partial quotients of finite continued fractions", Funct. Anal. Appl., 38:2 (2004), 79-87.

[42] А. В. Устинов, "О статистических свойствах конечных цепных дробей”, Труды по теории чисел, Зап. науч. сем. ПОМИ, 322, ПОМИ, СПб., 2005, 186-211; англ. пер.: A.V. Ustinov, "On the statistical properties of finite continued fractions", J. Math. Sci. (N. Y.), 137:2 (2006), 4722-4738.

[43] А. В. Устинов, "О статистиках Гаусса-Кузьмина для конечных цепных дробей", Фундамент. и прикл. матем., 11:6 (2005), 195-208; англ. пер.: А. V. Ustinov, "On Gauss-Kuz'min statistics for finite continued fractions", J. Math. Sci. (N. Y.), 146:2 (2007), 5771-5781.

[44] А.В. Устинов, "О числе решений сравнения $x y \equiv l(\bmod q)$ под графиком дважды непрерывно дифференцируемой функции", Алгебра и анализ, 20:5 (2008), 186-216; англ. пер.: A. V. Ustinov, "On the number of solutions of the congruence $x y \equiv l(\bmod q)$ under the graph of a twice continuously differentiable function", St. Petersburg Math. J., 20:5 (2009), 813-836.

[45] А. В. Устинов, "Спиновые цепочки и задача Арнольда о статистиках ГауссаКузьмина для квадратичных иррациональностей”, Матем. сб., 204:5 (2013), 143-160; англ. пер.: A.V. Ustinov, "Spin chains and Arnold's problem on the Gauss-Kuz'min statistics for quadratic irrationals", Sb. Math., 204:5 (2013), 762-779.

[46] H. Petersson, "Über eine Funktion von G. Lochs und die Diskriminante der elliptischen Funktionen", Monatsh. Math., 67:3 (1963), 243-258.

[47] А. В. Устинов, "О статистиках Гаусса-Кузьмина в коротких интервалах", Дальневост. матем. журн., 11:1 (2011), 93-98.

[48] G. J. Rieger, "Über die mittlere Schrittanzahl bei Divisionsalgorithmen", Math. Nachr., 82:1 (1978), 157-180.

[49] А. В. Устинов, "О среднем числе шагов в алгоритме Евклида с выбором минимального по модулю остатка", Матем. заметки, 85:1 (2009), 153-156; англ. пер.: A.V. Ustinov, "The mean number of steps in the Euclidean algorithm with least absolute-value remainders", Math. Notes, 85:1 (2009), 142-145.

[50] G. J. Rieger, "Ein Heilbronn-Satz für Kettenbrüche mit ungeraden Teilnennern", Math. Nachr., 101:1 (1981), 295-307.

[51] А.В. Устинов, "О среднем числе шагов в алгоритме Евклида с нечетными неполными частными", Матем. заметки, 88:4 (2010), 594-604; англ. пер.: A.V. Ustinov, "The mean number of steps in the Euclidean algorithm with odd partial quotients", Math. Notes, 88:4 (2010), 574-584. 
[52] F. P. Boca, J. Vandehey, "On certain statistical properties of continued fractions with even and with odd partial quotients", Acta Arith., 156:3 (2012), 201-221.

[53] Е. Н. Жабицкая, "Средняя длина приведенной регулярной непрерывной дроби”, Матем. сб., 200:8 (2009), 79-110; англ. пер.: E. N. Zhabitskaya, "The average length of reduced regular continued fractions", Sb. Math., 200:8 (2009), 1181-1214.

[54] Д. А. Фроленков, “Асимптотическое поведение первого момента для числа шагов в алгоритме Евклида по избытку и недостатку", Матем. сб., 203:2 (2012), 143-160; англ. пер.: D. A. Frolenkov, "Asymptotic behaviour of the first moment of the number of steps in the by-excess and by-deficiency Euclidean algorithms", $S b$. Math., 203:2 (2012), 288-305.

[55] D. E. Knuth, A. C. Yao, "Analysis of the subtractive algorithm for greatest common divisors", Proc. Natl. Acad. Sci. U.S.A., 72:12 (1975), 4720-4722.

[56] Е. Н. Жабицкая, "Среднее значение сумм неполных частных непрерывной дроби", Матем. заметки, 89:3 (2011), 472-476; англ. пер.: Е. N. Zhabitskaya, "Mean value of sums of partial quotients of continued fractions", Math. Notes, 89:3 (2011), 450-454.

[57] О. А. Горкуша, "О средней длине диагональных дробей Минковского", Дальневост. матем. журн., 11:1 (2011), 10-27.

[58] О. А. Горкуша, "О конечных цепных дробях специального вида", Чебышевский cб., 9:1(25) (2008), 80-107.

[59] F. P. Boca, R. N. Gologan, A. Zaharescu, "The statistics of the trajectory of a certain billiard in a flat two-torus", Comm. Math. Phys., 240:1-2 (2003), 53-73.

[60] F.P. Boca, A. Zaharescu, "The distribution of the free path lengths in the periodic two-dimensional Lorentz gas in the small-scatterer limit", Comm. Math. Phys., 269:2 (2007), 425-471.

[61] В.А. Быковский, А.В. Устинов, "Статистика траекторий частиц в однородной задаче Синая для двумерной решетки”, Функи. анализ и его прил., 42:3 (2008), 10-22; англ. пер.: V. A. Bykovskii, A. V. Ustinov, "The statistics of particle trajectories in the homogeneous Sinai problem for a two-dimensional lattice", Funct. Anal. Appl., 42:3 (2008), 169-179.

[62] В.А. Быковский, А. В. Устинов, "Статистика траекторий частиц в неоднородной задаче Синая для двумерной решетки", Изв. РАН. Сер. матем., 73:4 (2009), 17-36; англ. пер.: V. A. Bykovskii, A. V. Ustinov, "The statistics of particle trajectories in the inhomogeneous Sinai problem for a two-dimensional lattice", $I z v$. Math., 73:4 (2009), 669-688.

[63] F. P. Boca, C. Cobeli, A. Zaharescu, "Distribution of lattice points visible from the origin", Comm. Math. Phys., 213:2 (2000), 433-470.

[64] А. В. Устинов, "О распределении точек целочисленной решетки", Дальневост. матем. журн., 9:1-2 (2009), 176-181.

[65] Ж. Бургейн, Я.Г. Синай, "Предельное поведение больших чисел Фробениуса", УМH, 62:4(376) (2007), 77-90; англ. пер.: J. Bourgain, Ya. G. Sinai, "Limit behaviour of large Frobenius numbers", Russian Math. Surveys, 62:4 (2007), $713-725$.

[66] V. Shchur, Ya. Sinai, A. Ustinov, "Limiting distribution of Frobenius numbers for $n=3$ ", J. Number Theory, 129:11 (2009), 2778-2789.

[67] А.В. Устинов, "Решение задачи Арнольда о слабой асимптотике для чисел Фробениуса с тремя аргументами", Матем. сб., 200:4 (2009), 131-160; англ. пер.: A. V. Ustinov, "The solution of Arnold's problem on the weak asymptotics of Frobenius numbers with three arguments", Sb. Math., 200:4 (2009), 597-627. 
[68] А. В. Устинов, "О распределении чисел Фробениуса с тремя аргументами", Изв. РАН. Сер. матем., 74:5 (2010), 145-170; англ. пер.: А. V. Ustinov, "On the distribution of Frobenius numbers with three arguments", Izv. Math., 74:5 (2010), 1023-1049.

[69] Д. А. Фроленков, "Среднее значение чисел Фробениуса с тремя аргументами", Изв. РАН. Сер. матем., 76:4 (2012), 125-184; англ. пер.: D. A. Frolenkov, "The mean value of Frobenius numbers with three arguments", Izv. Math., 76:4 (2012), 760-819.

[70] W. B. Jurkat, J. W. Van Horne, "The proof of the central limit theorem for theta sums", Duke Math. J., 48:4 (1981), 873-885.

[71] W. B. Jurkat, J. W. Van Horne, "On the central limit theorem for theta series", Michigan Math. J., 29:1 (1982), 65-77.

[72] W.B. Jurkat, J. W. Van Horne, "The proof of the central limit theorem for theta sums", Duke Math. J., 48:4 (1981), 873-885.

[73] E. Demirci Akarsu, J. Marklof, "The value distribution of incomplete Gauss sums", Mathematika, 59:2 (2013), 381-398.

[74] V. Baladi, B. Vallée, "Euclidean algorithms are Gaussian", J. Number Theory, 110:2 (2005), 331-386.

[75] B. Vallée, "A unifying framework for the analysis of a class of Euclidean algorithms", LATIN 2000: theoretical informatics, Proceedings of the 4th Latin American Symposium (Punta del Este, April 10-14, 2000), Lecture Notes in Comput. Sci., 1776, Springer-Verlag, Berlin, 2000, 343-354.

[76] B. Vallée, "Dynamical analysis of a class of Euclidean algorithms", Theoret. Comput. Sci., 297:1-3 (2003), 447-486.

[77] J. Marklof, A. Strömbergsson, "The distribution of free path lengths in the periodic Lorentz gas and related lattice point problems", Ann. of Math. (2), 172:3 (2010), 1949-2033.

[78] J. Marklof, A. Strömbergsson, "The periodic Lorentz gas in the Boltzmann-Grad limit: asymptotic estimates", Geom. Funct. Anal., 21:3 (2011), 560-647.

[79] J. Marklof, A. Strömbergsson, "Free path lengths in quasicrystals", Comm. Math. Phys., 330:2 (2014), 723-755.

[80] J. Marklof, "The asymptotic distribution of Frobenius numbers", Invent. Math., 181:1 (2010), 179-207.

[81] I. Aliev, M. Henk, "Integer knapsacks: average behavior of the Frobenius numbers", Math. Oper. Res., 34:3 (2009), 698-705.

[82] I. Aliev, M. Henk, A. Hinrichs, "Expected Frobenius numbers", J. Combin. Theory Ser. A, 118:2 (2011), 525-531.

[83] A. Strömbergsson, "On the limit distribution of Frobenius numbers", Acta Arith., 152 (2012), 81-107.

[84] J. Marklof, "Limit theorems for theta sums", Duke Math. J., 97:1 (1999), 127-153.

[85] F. Cellarosi, "Limiting curlicue measures for theta sums", Ann. Inst. Henri Poincaré Probab. Statist., 47:2 (2011), 466-497.

[86] M. L. Kontsevich, Yu. M. Suhov, "Statistics of Klein polyhedra and multidimensional continued fractions", Pseudoperiodic topology, Amer. Math. Soc. Transl. Ser. 2, 197, Amer. Math. Soc., Providence, RI, 1999, 9-27.

[87] О.Н. Карпенков, "Об инвариантной мере Мёбиуса и распределении граней Гаусса-Кузьмина", Анализ и особенности. Часть 1, Сборник статей. K 70-летию со дня рождения академика Владимира Игоревича Арнольда, Тр. 
МИАН, 258, Наука, М., 2007, 79-92; англ. пер.: О. N. Karpenkov, "On an invariant Möbius measure and the Gauss-Kuzmin face distribution", Proc. Steklov Inst. Math., 258 (2007), 74-86.

[88] F. Klein, "Über eine geometrische Auffassung der gewöhnlichen Kettenbruchentwickelung", Nachr. Ges. Wiss. Göttingen Math.-Phys. Kl., 1895:3 (1895), 357-359.

[89] F. Klein, "Sur une représentation géométrique du développement en fraction continue ordinaire", Nouv. Ann. Math. (3), 15 (1896), 327-331.

[90] H. J.S. Smith, The collected mathematical papers, v.2, Clarendon Press, Oxford, 1894, vii+719 pp.

[91] H. Minkowski, "Zur Theorie der Kettenbrüche", Gesammelte Abhandlungen, v. 1, Druck und Verlag von B. G. Teubner, Leipzig, 1911, 278-292.

[92] В.А. Быковский, "Относительные минимумы решеток и вершины многогранников Клейна”, Функи. анализ и его прил., 40:1 (2006), 69-71; англ. пер.: V. A. Bykovskii, "Local minima of lattices and vertices of Klein polyhedra", Funct. Anal. Appl., 40:1 (2006), 56-57.

[93] О.Н.Герман, "Полиэдры Клейна и относительные минимумы решеток", Матем. заметки, 79:4 (2006), 546-552; англ. пер.: O. N. German, "Klein polyhedra and relative minima of lattices", Math. Notes, 79:4 (2006), 505-510.

[94] А. В. Устинов, "Минимальные системы векторов в трехмерных решетках и аналог теоремы Валена для трехмерных цепных дробей Минковского", Математика и информатика, $1, \mathrm{~K}$ 75-летию со дня рождения Анатолия Алексеевича Карацубы, Совр. пробл. матем., 16, МИАН, М., 2012, 103-128; англ. пер.: A. V. Ustinov, "Minimal vector systems in three-dimensional lattices and an analog of Vahlen's theorem for three-dimensional Minkowski continued fractions", Proc. Steklov Inst. Math., 280:, suppl. 2 (2013), S91-S116.

[95] М. О. Авдеева, В.А. Быковский, “Аналог теоремы Валена для совместных приближений пары чисел”, Матем. сб., 194:7 (2003), 3-14; англ. пер.: M. O. Avdeeva, V. A. Bykovskii, "An analogue of Vahlen's theorem for simultaneous approximations of a pair of numbers", Sb. Math., 194:7 (2003), 955-967.

[96] В.А. Быковский, О.А. Горкуша, "Минимальные базисы трехмерных полных решеток", Матем. сб., 192:2 (2001), 57-66; англ. пер.: V. A. Bykovskii, O. A. Gorkusha, "Minimal bases of three-dimensional complete lattices", Sb. Math., 192:2 (2001), 215-223.

[97] Ph. Furtwängler, M. Zeisel, "Zur Minkowskischen Parallelepipedapproximation", Monatsh. Math. Phys., 30:1 (1920), 177-198.

[98] P. M. Pepper, "Une application de la géométrie des nombres à une généralisation d'une fraction continue", Ann. Sci. École Norm. Sup. (3), 56 (1939), 1-70.

[99] S. Felsner, F. Zickfeld, "Schnyder woods and orthogonal surfaces", Discrete Comput. Geom., 40:1 (2008), 103-126.

[100] O. Karpenkov, A. Ustinov, "Geometry of Minkowski-Voronoi tessellations of the plane", 2014, 30 pp., arXiv: 1407.0135.

[101] H. Davenport, "On the product of three homogeneous linear forms. IV", Proc. Cambridge Philos. Soc., 39:1 (1943), 1-21.

[102] H. P. F. Swinnerton-Dyer, "On the product of three homogeneous linear forms", Acta Arith., 18 (1971), 371-385.

[103] J. W.S. Cassels, H.P. F. Swinnerton-Dyer, "On the product of three homogeneous linear forms and indefinite ternary quadratic forms", Philos. Trans. Roy. Soc. London. Ser. A, 248:940 (1955), 73-96. 
[104] J.W.S. Cassels, An introduction to the geometry of numbers, Corrected reprint of the 1971 ed., Classics Math., Springer-Verlag, Berlin, 1997, viii+344 pp.; рус. пер. 1-го изд.: Дж. В. Касселс, Введение в геометрию чисел, Мир, М., 1965, 421 с.

[105] J. Buchmann, "A generalization of Voronoi's unit algorithm. I", J. Number Theory, 20:2 (1985), 177-191.

[106] J. Buchmann, "A generalization of Voronoi's unit algorithm. II", J. Number Theory, 20:2 (1985), 192-209.

[107] J. Buchmann, "The computation of the fundamental unit of totally complex quartic orders", Math. Comp., 48:117 (1987), 39-54.

[108] J. Buchmann, "On the computation of units and class numbers by a generalization of Lagrange's algorithm", J. Number Theory, 26:1 (1987), 8-30.

[109] T. W. Cusick, "Diophantine approximation of ternary linear forms", Math. Comp., 25:113 (1971), 163-180.

[110] T. W. Cusick, "The two-dimensional Diophantine approximation constant. II", $P a-$ cific J. Math., 105:1 (1983), 53-67.

[111] H. C. Williams, G. Cormack, E. Seah, "Calculation of the regulator of a pure cubic field", Math. Comp., 34:150 (1980), 567-611.

[112] H. C. Williams, "Some results concerning Voronoi's continued fraction over $\mathscr{Q}(\sqrt[3]{D})$ ", Math. Comp., 36:154 (1981), 631-652.

[113] В. А. Быковский, “Теорема Валена для двумерных подходящих дробей”, Матем. заметки, 66:1 (1999), 30-37; англ. пер.: V. A. Bykovskii, "Vahlen theorem for two-dimensional convergents", Math. Notes, 66:1 (1999), 24-29.

[114] М.О. Авдеева, В.А. Быковский, "Уточнение теоремы Валена для базисов Минковского трехмерных решеток", Матем. заметки, 79:2 (2006), 163-168; англ. пер.: M. O. Avdeeva, V. A. Bykovskii, "Refinement of Vahlen's theorem for Minkowski bases of three-dimensional lattices", Math. Notes, 79:2 (2006), 151-156.

[115] А. В. Устинов, "К трехмерной теореме Валена", Матем. заметки, 95:1 (2014), 154-156; англ. пер.: А. V. Ustinov, "On the three-dimensional Vahlen theorem", Math. Notes, 95:1 (2014), 136-138.

[116] G. Ramharter, "On Mordell's inverse problem in dimension three", J. Number Theory, 58:2 (1996), 388-415.

[117] В. А. Быковский, "О погрешности теоретико-числовых квадратурных формул", Чебышевский сб., 3:2(4) (2002), 27-33.

[118] В. А. Быковский, "О погрешности теоретико-числовых квадратурных формул", Докл. РАН, 389:2 (2003), 154-155; англ. пер.: V. A. Bykovskii, "On the error of number-theoretic quadrature formulas", Dokl. Math., 67:2 (2003), 175-176.

[119] В.А. Быковский, “Алгоритм вычисления локальных минимумов решеток”, Докл. РАН, 399:5 (2004), 587-589.

[120] В. А. Быковский, С. В. Гассан, "Алгоритм вычисления локальных минимумов целочисленных решеток и его приложения", Вестн. ТОГУ, 2011, № 1(20), 39-48.

[121] О. А. Горкуша, Н. М. Добровольский, "Об оценках гиперболической дзета-функции решеток", Чебышевский сб., 6:2(14) (2005), 129-137.

[122] В. А. Быковский, "Отклонение сеток Коробова", Изв. РАН. Сер. матем., 76:3 (2012), 19-38; англ. пер.: V. A. Bykovskii, "The discrepancy of the Korobov lattice points", Izv. Math., 76:3 (2012), 446-465.

[123] O. Karpenkov, Geometry of continued fractions, Algorithms Comput. Math., 26, Springer, Heidelberg, 2013, xviii+405 pp. 
[124] А. В. Устинов, "О точках модулярной гиперболы под графиком линейной функции", Матем. заметки, 97:2 (2015), 296-301; англ. пер.: А. V. Ustinov, "On points of the modular hyperbola under the graph of a linear function", Math. Notes, 97:2 (2015), 284-288.

[125] A. Weil, "On some exponential sums", Proc. Natl. Acad. Sci. U.S.A., 34 (1948), 204-207.

[126] T. Estermann, "On Kloosterman's sum”, Mathematika, 8:1 (1961), 83-86.

[127] S. R. Finch, Mathematical constants, Encyclopedia Math. Appl., 94, Cambridge Univ. Press, Cambridge, 2003, xx+602 pp.

\section{Алексей Владимирович Устинов} (Alexey V. Ustinov)

Хабаровское отделение Института прикладной математики ДВО РАН

E-mail: ustinov@iam.khv.ru
Поступила в редакцию

04.12 .2014 